quatrième série-tome 43 fascicule 1 janvier-février 2010

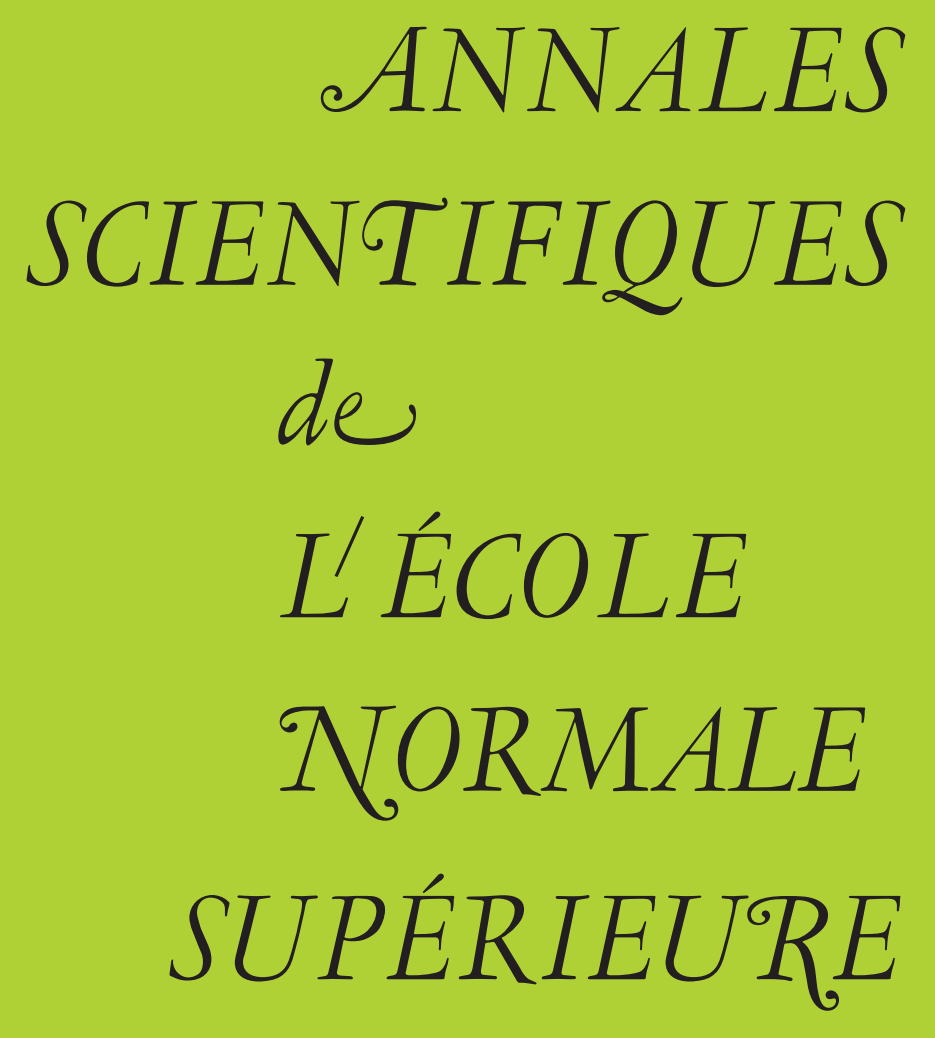

Daniel ALLCOCK \& James A. CARLSON \& Domingo TOLEDO Hyperbolic geometry and moduli of real cubic surfaces 
Ann. Scient. Éc. Norm. Sup.

$4^{\mathrm{e}}$ série, t. 43,2010, p. 69 à 115

\title{
HYPERBOLIC GEOMETRY AND MODULI OF REAL CUBIC SURFACES
}

\author{
By Daniel Allcock, James A. CARLSON \\ AND Domingo TOLEDO
}

\begin{abstract}
Let $\mathcal{M}_{0}^{\mathbb{R}}$ be the moduli space of smooth real cubic surfaces. We show that each of its components admits a real hyperbolic structure. More precisely, one can remove some lowerdimensional geodesic subspaces from a real hyperbolic space $H^{4}$ and form the quotient by an arithmetic group to obtain an orbifold isomorphic to a component of the moduli space. There are five components. For each we describe the corresponding lattices in $\mathrm{PO}(4,1)$. We also derive several new and several old results on the topology of $\mathcal{M}_{0}^{\mathbb{R}}$. Let $\mathcal{M}_{s}^{\mathbb{R}}$ be the moduli space of real cubic surfaces that are stable in the sense of geometric invariant theory. We show that this space carries a hyperbolic structure whose restriction to $\mathcal{M}_{0}^{\mathbb{R}}$ is that just mentioned. The corresponding lattice in $\mathrm{PO}(4,1)$, for which we find an explicit fundamental domain, is nonarithmetic.
\end{abstract}

RÉSUMÉ. - On note $\mathcal{M}_{0}^{\mathbb{R}}$ l'espace des modules des surfaces cubiques réelles lisses. Nous montrons que chacune de ses composantes admet une structure hyperbolique réelle. Plus précisément, en enlevant de l'espace hyperbolique réel $H^{4}$ certaines sous-variétés totalement géodésiques de dimension inférieure, puis en prenant le quotient par un groupe arithmétique, on obtient une orbifold isomorphe à une composante de l'espace des modules. Il y a cinq composantes. Nous décrivons le réseau de $P O(4,1)$ qui correspond à chacune d'entre elles. Nous démontrons également quelques résultats sur la topologie de $\mathcal{M}_{0}^{\mathbb{R}}$, dont certains sont nouveaux. On note $\mathcal{M}_{s}^{\mathbb{R}}$ l'espace des modules des surfaces cubiques réelles qui sont stables au sens de la théorie géométrique des invariants. Nous montrons que cet espace admet une structure hyperbolique dont la restriction à $\mathcal{M}_{0}^{\mathbb{R}}$ est celle évoquée ci-dessus. Nous décrivons un domaine fondamental pour le réseau correspondant de $P O(4,1)$, qui s'avère être non arithmétique.

\section{Introduction}

The purpose of this paper is to study the geometry and topology of the moduli space of real cubic surfaces in $\mathbb{R} P^{3}$. It is a classical fact, going back to Schläfli [31, 33] and Klein [21], that the moduli space of smooth real cubic surfaces has five connected components.

First author partly supported by NSF grants DMS 0070930, DMS-0231585 and DMS-0600112. Second and third authors partly supported by NSF grants DMS 9900543, DMS-0200877 and DMS-0600816. The second author thanks the Clay Mathematics Institute for its support. 
We show in this paper that each of these components has a real hyperbolic structure that we compute explicitly, both in arithmetic and in geometric terms. We use this geometric structure to compute, to a large extent, the topology of each component. These structures are not complete. We also prove a more subtle result, that the moduli space of stable real cubic surfaces has a real hyperbolic structure, which is complete, and that restricts, on each component of the moduli space of smooth surfaces, to the (incomplete) structures just mentioned. The most surprising fact to us is that the resulting discrete group of isometries of hyperbolic space is not arithmetic.

To describe our results, we use the following notation. We write $\mathscr{C}$ for the space of nonzero cubic forms with complex coefficients in 4 variables, $\Delta$ for the discriminant locus (forms where all partial derivatives have a common zero), $\mathscr{C}_{0}$ for the space $\mathscr{C}-\Delta$ of forms that define a smooth hypersurface in $\mathbb{C} P^{3}$, and $\mathscr{C}_{s}$ for the space of forms that are stable in the sense of geometric invariant theory for the action of $\operatorname{GL}(4, \mathbb{C})$ on $\mathscr{C}$. It is classical that these are the forms that define a cubic surface which is either smooth or has only nodal singularities [18, $\S 19]$.

We denote all the corresponding real objects with a superscript $\mathbb{R}$. Thus $\mathscr{C}^{\mathbb{R}}$ denotes the space of non-zero cubic forms with real coefficients, and $\Delta^{\mathbb{R}}, \mathscr{C}_{0}^{\mathbb{R}}$ and $\mathscr{C}_{s}^{\mathbb{R}}$ the intersection with $\mathscr{C}^{\mathbb{R}}$ of the corresponding subspaces of $\mathscr{C}$. We will also use the prefix $P$ for the corresponding projective objects, thus $P \mathscr{C}^{\mathbb{R}} \cong \mathbb{R} P^{19}$ is the projective space of cubic forms with real coefficients, and $P \Delta^{R}, P \mathscr{C}_{0}^{\mathbb{R}}, P \mathscr{C}_{s}^{\mathbb{R}}$ are the images of the objects just defined. The group $\operatorname{GL}(4, \mathbb{R})$ acts properly on $\mathscr{C}_{0}^{\mathbb{R}}$ and $\mathscr{C}_{s}^{\mathbb{R}}$ (equivalently, $\operatorname{PGL}(4, \mathbb{R})$ acts properly on $P \mathscr{C}_{0}^{\mathbb{R}}$ and $\left.P C_{s}^{\mathbb{R}}\right)$ and we write $\mathcal{M}_{0}^{\mathbb{R}}$ and $\mathcal{M}_{s}^{\mathbb{R}}$ for the corresponding quotient spaces, namely the moduli spaces of smooth and of stable real cubic surfaces.

The space $P \Delta^{\mathbb{R}}$ has real codimension one in $P \mathscr{C}^{\mathbb{R}}$, its complement $P \mathscr{C}_{0}^{\mathbb{R}}$ has five connected components, and the topology of a surface in each component is classically known [21, 33, 34]. We label the components $P \mathscr{C}_{0, j}^{\mathbb{R}}$, for $j=0,1,2,3,4$, choosing the indexing so that a surface in $P \mathscr{C}_{0, j}^{\mathbb{R}}$ is topologically a real projective plane with $3-j$ handles attached (see table 1.1; the case of -1 many handles means the disjoint union $\mathbb{R} P^{2} \sqcup S^{2}$ ). It follows that the moduli space $\mathcal{M}_{0}^{\mathbb{R}}$ has five connected components, $\mathcal{M}_{0, j}^{\mathbb{R}}$, for $j=0,1,2,3,4$. We can now state our first theorem:

TheOrem 1.1. - For each $j=0, \ldots, 4$ there is a union $\mathcal{H}_{j}$ of two- and three-dimensional geodesic subspaces of the four-dimensional real hyperbolic space $H^{4}$ and an isomorphism of real analytic orbifolds

$$
\mathcal{M}_{0, j}^{\mathbb{R}} \cong P \Gamma_{j}^{\mathbb{R}} \backslash\left(H^{4}-\mathcal{H}_{j}\right) .
$$

Here $P \Gamma_{j}^{\mathbb{R}}$ is the projectivized group of integer matrices which are orthogonal with respect to the quadratic form obtained from the diagonal form $[-1,1,1,1,1]$ by replacing the last $j$ of the 1 's by 3 's.

The real hyperbolic structure on the component $\mathcal{M}_{0,0}^{\mathbb{R}}$ has been studied by Yoshida [41]. The other cases are new.

The space $P \mathscr{C}_{s}^{\mathbb{R}}$ is connected, since it is obtained from the manifold $P \mathscr{C}^{\mathbb{R}}$ by removing a subspace of codimension two (part of the singular set of $P \Delta^{\mathbb{R}}$ ). Thus the moduli space $M_{s}^{\mathbb{R}}$ is connected. We have the following uniformization theorem for this space: 
TheOREM 1.2. - There are a nonarithmetic lattice $P \Gamma^{\mathbb{R}} \subset P O(4,1)$ and a homeomorphism

$$
\mathcal{M}_{s}^{\mathbb{R}} \cong P \Gamma^{\mathbb{R}} \backslash H^{4} .
$$

Moreover, there is a $P \Gamma^{\mathbb{R}}$-invariant union of two-and three-dimensional geodesic subspaces $\mathcal{H}^{\prime}$ of $\mathrm{H}^{4}$ so that this homeomorphism restricts to an isomorphism of real analytic orbifolds,

$$
\mathcal{M}_{0}^{\mathbb{R}} \cong P \Gamma^{\mathbb{R}} \backslash\left(H^{4}-\mathcal{H}^{\prime}\right) .
$$

To our knowledge this is the first appearance of a non-arithmetic lattice in a moduli problem for real varieties. Observe that the group $P \Gamma^{\mathbb{R}}$ uniformizes a space assembled from arithmetic pieces much in the spirit of the construction by Gromov and Piatetskii-Shapiro of non-arithmetic lattices in real hyperbolic space. We thus view this theorem as an appearance "in nature" of their construction.

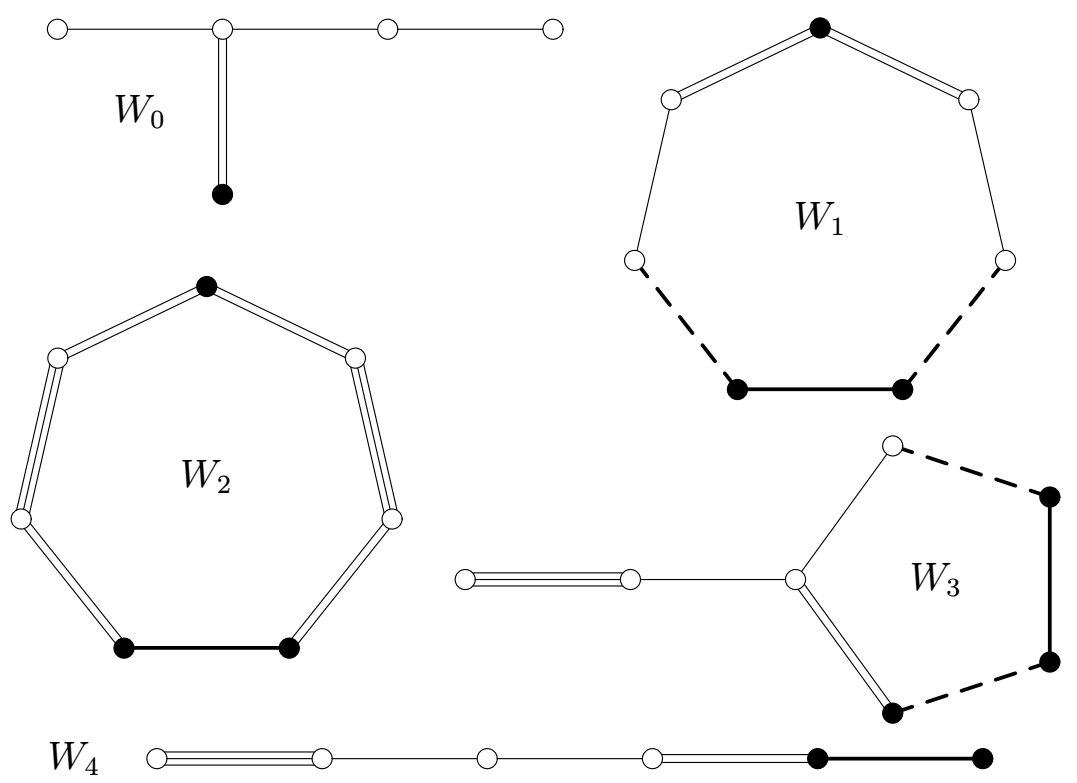

FigURE 1.1. Coxeter polyhedra for the reflection subgroups $W_{j}$ of $P \Gamma_{j}^{\mathbb{R}}$. The blackened nodes and triple bonds correspond to faces of the polyhedra that represent singular cubic surfaces. See the text for the explanation of the edges.

We obtain much more information about the groups $P \Gamma_{j}^{\mathbb{R}}$ and $P \Gamma^{\mathbb{R}}$ than we have stated here. Section 5 gives an arithmetic description of each $P \Gamma_{j}^{\mathbb{R}}$ and shows that they are essentially Coxeter groups. (Precisely: they are Coxeter groups for $j=0,3,4$ and contain a Coxeter subgroup of index 2 if $j=1,2$.) We use Vinberg's algorithm to derive their Coxeter diagrams and consequently their fundamental domains. So we have a very explicit geometric description of the groups $P \Gamma_{j}^{\mathbb{R}}$. The results are summarized in Fig. 1.1. In these diagrams the nodes represent facets of the polyhedron, and two facets meet at an angle of $\pi / 2, \pi / 3, \pi / 4$ or $\pi / 6$, or are parallel (meet at infinity) or are ultraparallel, if the number of bonds between the two corresponding nodes is respectively $0,1,2,3$, or a heavy or dashed line. See Section 5 for more details. 


\begin{tabular}{ccccc}
$j$ & $\begin{array}{c}\text { Topology } \\
\text { of Surface }\end{array}$ & Real Lines & $\begin{array}{c}\text { Real Tritang. } \\
\text { Planes }\end{array}$ & Monodromy on Lines \\
\hline 0 & $\mathbb{R} P^{2} \# 3 T^{2}$ & 27 & 45 & $A_{5}$ \\
1 & $\mathbb{R} P^{2} \# 2 T^{2}$ & 15 & 15 & $S_{3} \times S_{3}$ \\
2 & $\mathbb{R} P^{2} \# T^{2}$ & 7 & 5 & $(\mathbb{Z} / 2)^{3} \rtimes \mathbb{Z} / 2$ \\
3 & $\mathbb{R} P^{2}$ & 3 & 7 & $S_{4}$ \\
4 & $\mathbb{R} P^{2} \sqcup S^{2}$ & 3 & 13 & $S_{4}$ \\
\hline
\end{tabular}

TABLE 1.1. The classical results on the components of the moduli space of real cubic surfaces. The components are indexed by $j$ according to our conventions. The third item in the last column corrects an error of Segre.

$\left.\begin{array}{ccccc}j & \text { Euler } & \text { Volume } & \text { Fraction } & \pi_{1}^{\text {orb }}\left(\mathcal{M}_{0, j}^{\mathbb{R}}\right) \\ \hline 0 & 1 / 1920 & .00685 & 2.03 \% & S_{5} \\ 1 & 1 / 288 & .04569 & 13.51 \% & \left(S_{3} \times S_{3}\right) \rtimes \mathbb{Z} / 2 \\ 2 & 5 / 576 & .11423 & 33.78 \% & \left(D_{\infty} \times D_{\infty}\right) \rtimes \mathbb{Z} / 2 \\ 3 & 1 / 96 & .13708 & 40.54 \% \\ 4 & 1 / 384 & .03427 & 10.14 \%\end{array}\right\} \circ-\infty \quad 0$

TABLE 1.2. The orbifold Euler characteristic, volume, fraction of total volume, and orbifold fundamental groups of the moduli spaces $\mathcal{M}_{0, j}^{\mathbb{R}}$. See Theorem 7.1 for the notation.

The group $P \Gamma^{\mathbb{R}}$ is not a Coxeter group (even up to finite index) but we find that a subgroup of index two has a fundamental domain that is a Coxeter polyhedron. We describe this polyhedron explicitly in Section 12, thus we have a concrete geometric description of $P \Gamma^{\mathbb{R}}$, and we also find a representation of this group by matrices with coefficients in $\mathbb{Z}[\sqrt{3}]$.

Much of the classical theory of real cubic surfaces, as well as new results, are encoded in these Coxeter diagrams. The new results are our computation of the groups $\pi_{1}^{\text {orb }}\left(\mathcal{M}_{0, j}^{\mathbb{R}}\right)$ (see table 1.2) and our proof that each $\mathcal{M}_{0, j}^{\mathbb{R}}$ has contractible universal cover. These results appear in Section 7, where we describe the topology of the spaces $M_{0, j}^{\mathbb{R}}$. As an application to the classical theory, we re-compute the monodromy representation of $\pi_{1}\left(P \mathscr{C}_{0, j}^{\mathbb{R}}\right)$ on the configuration of lines on a cubic surface, which was first computed by Segre in his treatise [34]. We confirm four of his computations and correct an error in the remaining one (the case $j=2$ ). See the last column of table 1.1 and Section 8 for details. We also compute the hyperbolic volume of each component in Section 9. The results are summarized in table 1.2.

Our methods are based on our previous work on the complex hyperbolic structure of the moduli space of complex cubic surfaces [1]. We proved that this moduli space $\mathcal{M}_{s}$ is isomorphic to the quotient $P \Gamma \backslash \mathbb{C} H^{4}$ of complex hyperbolic 4-space $\mathbb{C} H^{4}$ by the lattice 
$P \Gamma=P U(4,1, \mathcal{E})$ in $P U(4,1)$, where $\mathcal{E}$ is the ring of integers in $\mathbb{Q}(\sqrt{-3})$. We also showed that there is an infinite hyperplane arrangement $\mathcal{H}$ in $\mathbb{C} H^{4}$ which is $P \Gamma$-invariant and corresponds to the discriminant. Thus there is also an identification of the moduli space $\mathcal{M}_{0}$ of smooth cubic surfaces with the quotient $P \Gamma \backslash\left(\mathbb{C} H^{4}-\mathcal{H}\right)$. The natural map $\mathcal{M}_{0}^{\mathbb{R}} \rightarrow \mathcal{M}_{0}$ (which is finite to one but not injective) allows us to give a real hyperbolic structure on $\mathcal{M}_{0}^{\mathbb{R}}$ and thus prove Theorem 1.1. The essence of the proof appears in Section 3, with refinements in Sections 4-6. Theorem 1.2 is considerably more subtle, and does not follow simply from the corresponding map $\mathcal{M}_{s}^{\mathbb{R}} \rightarrow \mathcal{M}_{s}$. Its proof occupies Sections 11-13.

The subject of real cubic surfaces has a long and fascinating history. It was Schläfli who first discovered that there are five distinct types of real smooth cubic surfaces, distinguished by the numbers of real lines and real tritangent planes; see table 1.1. He summarized his results in his 1858 paper [31]. In a later paper [33], followed by corrections after correspondence with Klein, Schläfli also determined the topology (more precisely, the "connectivity") of a surface in each of his five types. In particular he showed that the topology is constant in each type. It is clear from these two later papers that he had a mental picture of the adjacency relationship of the components and how the topology of a smooth surface changes by "surgery" in going from one class to a neighboring one by crossing the discriminant.

Even though it is not closely related to the present discussion, we should also mention Schläfli's monumental paper [32] where he classifies all possible real cubic surfaces with all their possible singularities. One particular fact is that a cubic surface can have at most four nodes, and that there is a real cubic surface with four real nodes.

In 1873 Klein [21] gave a very clear picture of the space of smooth real cubic surfaces, of how the discriminant separates it into components, and of the topology of a surface in each of Schläfli's classes. He obtained all cubic surfaces by deforming the four-real-nodal cubic surface, and in this way he could see the topology of each nodal or smooth real cubic surface. $\mathrm{He}$ also proved that the space of smooth real cubic surfaces has five connected components and that Schläfli's classes coincide with the components. The proof required the knowledge of the space of nodal cubic surfaces, and also some information on cuspidal ones. Klein was not satisfied with the arguments in his original 1873 paper [21], and made corrections and substantial amplifications in the version published in his collected works in 1922.

A proof, by Klein's method, that $P \mathscr{C}_{0}^{\mathbb{R}}$ has five connected components is given in $\$ 24$ of Segre's book [34]. Segre also determines in \$64-68 the topology of a surface in each component by studying how the surface is divided into cells by its real lines. This book also contains a wealth of information about the real cubic surfaces, including the monodromy representations mentioned above, and much detailed information on the various configurations of lines.

Since the classification of real cubic surfaces is a special case of the classification of real cubic hypersurfaces, we also review the history of the latter. The earliest work is Newton's classification of real cubic curves [28, 29]. But in dimensions higher than two the classification results are very recent. We have Krasnov's classification of real cubic threefolds [22], which is based on Klein's method of determining the discriminant and then deforming away to see the components. Finashin and Kharlamov have two papers on the classification of real cubic fourfolds. The first one [12] is based partly on complex period maps (to study the discriminant) and partly on Klein's method. The second [13] is based on the surjectivity of 
the period map for complex cubic fourfolds recently proved by Laza and Looijenga [23, 24]. We are not aware of any classification of real cubic hypersurfaces beyond dimension four.

Our approach to $\mathcal{M}_{0}^{\mathbb{R}}$, namely studying a complex period map and its interaction with antiholomorphic involutions, has been used before, for instance, by Kharlamov in the study of quartic surfaces [19], by Nikulin for all the families of K3 surfaces [30] and by Gross and Harris for curves and abelian varieties [16]. In particular, Nikulin parametrizes the different connected components of real K3 surfaces by quotients of products of real hyperbolic spaces by discrete groups generated by reflections. Vinberg's algorithm has been used by Kharlamov [20] and more recently by Finashin and Kharlamov [12] to study the topology of some real moduli spaces.

Many authors, in addition to the ones already cited, have studied moduli of real algebraic varieties in terms of Coxeter diagrams or in terms of the action of complex conjugation on homology. We would like to mention the work of Degtyarev, Itenberg and Kharlamov on Enriques surfaces [10] and of Moriceau on nodal quartic surfaces [27]. There is considerable literature on moduli of $n$-tuples in $\mathbb{R} P^{1}$; see for example [36], [40], [5] and the papers they cite. Chu's paper [8] gives a real hyperbolic cone manifold structure to the moduli space of stable real octuples in $P^{1}$.

We have two expository articles [3, 4] that develop the ideas of this paper less formally, and in the context of related but simpler moduli problems, for which the moduli space has dimension $\leq 3$. The lower dimension means that all the fundamental domains can be visualized directly. The results of this paper were announced in [2].

We would like to thank János Kollár for helpful discussions at the early stages of this work. And we would like to thank the referee for numerous constructive comments that have greatly improved our exposition.

\section{Moduli of complex cubic surfaces}

We record here the key constructions and results of our description [1] of the moduli space of smooth complex cubic surfaces as a quotient of an open dense subset of complex hyperbolic 4-space $\mathbb{C} H^{4}$. Everything we need from that paper appears here. Only the results through Theorem 2.4 are required for Section 3 (moduli of smooth real surfaces). The last part of this section is needed for Sections 4 and 8 (relations with classical work). For background on singular complex cubic surfaces, see Section 10.

\subsection{Notation}

The key object is the moduli space $\mathcal{M}_{0}$ of smooth cubic surfaces in $\mathbb{C} P^{3}$, which we now define. As in [1, (2.1)], and in the introduction, let $\mathscr{C}$ be the space of all nonzero cubic forms in 4 complex variables, $\Delta$ the discriminant locus, and $\mathscr{C}_{0}=\mathscr{C}-\Delta$ the set of forms defining smooth cubic surfaces. We take $g \in \mathrm{GL}(4, \mathbb{C})$ to act in the usual way on the left on $\mathbb{C}^{4}$, and on the left on $\mathscr{C}$ by

$$
(g . F)(X)=F\left(g^{-1} X\right)
$$

for $X \in \mathbb{C}^{4}$. This is as in [1, (2.17)]. The action on $\mathscr{C}$ is not faithful: the subgroup acting trivially is $D=\{I, \omega I, \bar{\omega} I\}$ where $\omega=e^{2 \pi i / 3}$ is a primitive cube root of unity fixed 
throughout the paper. We write $G$ for the group $\operatorname{GL}(4, \mathbb{C}) / D$ acting effectively, and define $\mathcal{M}_{0}$ as $G \backslash \mathscr{C}_{0}$. As discussed in [1, (2.18) and (3.1)], $G$ acts properly on $\mathscr{C}_{0}$, so the moduli space $\mathcal{M}_{0}$ is a complex-analytic orbifold in a natural way.

\subsection{Framed cubic surfaces}

The relation between $\mathcal{M}_{0}$ and $\mathbb{C} H^{4}$ depends on a (multi-valued) period map whose construction involves cubic threefolds. Briefly, we first construct the space $\mathcal{F}_{0}$ of "framed smooth cubic forms", a certain covering space of $\mathscr{C}_{0}$, and then we define a (single-valued) period map $g: \mathcal{F}_{0} \rightarrow \mathbb{C} H^{4}$. This map is equivariant with respect to the deck group of the cover. Taking the quotient by this action gives a period map from $\mathscr{C}_{0}$ to a quotient of $\mathbb{C} H^{4}$, and this map factors through $\mathcal{M}_{0}$. It takes some work to define $\mathcal{F}_{0}$, so we begin with that.

If $F \in \mathscr{C}$ then we write $S$ for the surface it defines in $\mathbb{C} P^{3}$ and $T$ for the threefold in $\mathbb{C} P^{4}$ defined by

$$
Y^{3}-F\left(X_{0}, \ldots, X_{3}\right)=0 .
$$

Whenever we have a form $F$ in mind, we implicitly define $S$ and $T$ in this way. $T$ is the 3-fold cyclic covering of $\mathbb{C} P^{3}$ with ramification along $S$. We call it the cyclic cubic threefold associated to $F$. We define $\sigma \in \mathrm{GL}(5, \mathbb{C})$ by

$$
\sigma\left(X_{0}, \ldots, X_{3}, Y\right)=\left(X_{0}, \ldots, X_{3}, \omega Y\right) .
$$

It generates the deck group of $T$ over $\mathbb{C} P^{3}$. All the notation of this paragraph is from $[1,(2.1)]$.

Now suppose $F \in \mathscr{C}_{0}$. Then it is easy to see that $T$ is smooth. In [1, (2.2)] we show that $H^{3}(T, \mathbb{Z}) \cong \mathbb{Z}^{10}$ and that $\sigma$ fixes no element of this cohomology group (except 0 ). Because there are no $\sigma$-invariant elements, we may regard $H^{3}(T, \mathbb{Z})$ as a module over the Eisenstein integers $\mathscr{E}:=\mathbb{Z}[\omega]$, with $\omega$ acting as $\left(\sigma^{*}\right)^{-1}$. This gives a free $\mathcal{E}$-module of rank 5 , which we call $\Lambda(T)$. It is a key ingredient in the rest of the construction. $(\operatorname{In}[1,(2.2)]$ we took $\omega$ to act as $\sigma^{*}$, but unfortunately this made the period map antiholomorphic rather than holomorphic, as discussed in the note added in proof.)

Combining the action of $\sigma$ with the natural symplectic form $\Omega$ on $H^{3}(T, \mathbb{Z})$ gives an $\mathcal{E}$-valued Hermitian form on $\Lambda(T)$, defined by

$$
h(x, y)=\frac{1}{2}[\Omega(\theta x, y)+\theta \Omega(x, y)] .
$$

Here and throughout the paper, $\theta$ represents the Eisenstein integer $\omega-\bar{\omega}=\sqrt{-3}$; in particular, the first $\theta$ in (2.4) is the action of $\theta$ on $\Lambda(T)$, namely $\left(\sigma^{*}\right)^{-1}-\sigma^{*}$. This definition of $h$ is from [1, (2.3)], except that the sign is changed because of the change of $\delta$-module structures. The fact that $h$ is $\mathscr{E}$-valued, $\mathcal{E}$-linear in its first coordinate and $\mathcal{E}$-antilinear in its second is part of [1, Lemma 4.1]. Finally, in [1, (2.7)] we show that $\Lambda(T)$ is isometric to the lattice $\Lambda:=\delta^{4,1}$, meaning the free module $\delta^{5}$ equipped with the Hermitian form

$$
h(x, y)=-x_{0} \bar{y}_{0}+x_{1} \bar{y}_{1}+\cdots+x_{4} \bar{y}_{4} .
$$

Even though $\Lambda(T)$ is isometric to $\Lambda$, there is no preferred isometry, so we must treat them all equally. So we define a framing of $F \in \mathscr{C}_{0}$ as a projective equivalence class $[i]$ of $\mathcal{E}$-linear isometries $i: \Lambda(T) \rightarrow \Lambda$; thus $[i]=\left[i^{\prime}\right]$ just if $i$ and $i^{\prime}$ differ by multiplication by a unit of $\mathcal{E}$. A framed smooth cubic form is a pair $(F,[i])$ with $F \in \mathscr{C}_{0}$ and $[i]$ a framing of it, and $\mathcal{F}_{0}$ 
denotes the family of all framed smooth cubic forms. Usually we blur the distinction between $i$ and $[i]$; the main reason for introducing the equivalence relation is to make the action of $G$ on $\mathcal{F}_{0}$ well-defined (see below). In [1, (3.9)] we defined a natural complex manifold structure on $\mathcal{F}_{0}$, for which the obvious projection $\mathcal{F}_{0} \rightarrow \mathscr{C}_{0}$ is a holomorphic covering map, and we proved the following.

TheOREM $2.1([1,(3.9)]) .-\mathcal{F}_{0}$ is connected.

\subsection{Group actions on the space of framed surfaces}

The deck group for $\mathcal{F}_{0} \rightarrow \mathscr{C}_{0}$ is obviously $P \operatorname{Aut}(\Lambda)$. We will write $\Gamma$ for Aut $\Lambda$ and $P \Gamma$ for $P A u t(\Lambda)$. The meaning of $P \Gamma$ is the same as in [1], but the meaning of $\Gamma$ is slightly different. There, $\Gamma$ was the linear monodromy group, defined precisely in $[1,(2.11)]$, and it was proved in [1, Theorem 2.14] that Aut $\Lambda=\Gamma \times\{ \pm I\}$, so that PAut $\Lambda=P \Gamma$. Since the precise linear monodromy group will not be needed here, it will be convenient to use the abbreviation $\Gamma$ for Aut $\Lambda$.

As in [1, (3.9)] we write the action of $P \Gamma$ on $\mathcal{F}_{0}$ explicitly by

$$
\gamma \cdot(F,[i])=(F,[\gamma \circ i]),
$$

and define an action of $G$ on $\mathcal{F}_{0}$ as follows. Any $h \in \mathrm{GL}(4, \mathbb{C})$ acts on $\mathbb{C}^{5}$ by

$$
h\left(X_{0}, \ldots, X_{3}, Y\right)=\left(h\left(X_{0}, \ldots, X_{3}\right), Y\right) .
$$

If $(F,[i]) \in \mathcal{F}_{0}$ then $h$ carries the points of the threefold $T_{F}$ defined by (2.2) to those of the one $T_{h F}$ defined by the same formula with $h F$ replacing $F$. So $h$ induces an isometry $h^{*}: \Lambda\left(T_{h F}\right) \rightarrow \Lambda\left(T_{F}\right)$. Therefore $i \circ h^{*}$ is an isometry $\Lambda\left(T_{h F}\right) \rightarrow \Lambda$, so $\left(h F,\left[i \circ h^{*}\right]\right) \in \mathcal{F}_{0}$. We may therefore define an action of $\mathrm{GL}(4, \mathbb{C})$ on $\mathcal{F}_{0}$ by

$$
h .(F,[i]):=\left(h . F,\left[i \circ h^{*}\right]\right)=\left(F \circ h^{-1},\left[i \circ h^{*}\right]\right) .
$$

This action factors through $G$ because $\omega I \in \mathrm{GL}(4, \mathbb{C})$ fixes every $F$ and acts on $\mathbb{C} P^{4}$ in the same way as a power of $\sigma$. That is, $\omega I$ sends every $\Lambda(T)$ to itself by a scalar, so it fixes every framing.

As in [1, (2.18)] we define the moduli space of framed cubic surfaces $\mathcal{M}_{0}^{f}$ as $G \backslash \mathcal{F}_{0}$. It is an analytic space because $G$ acts properly on $\mathcal{F}_{0}$ (since it does on $\mathscr{C}_{0}$ ). But more is true:

Theorem 2.2 ([1, Lemma 3.14]). - The action of $G$ on $\mathcal{F}_{0}$ is free, so $\mathcal{M}_{0}^{f}$ is a complex manifold.

\subsection{The period map}

Having described $\mathcal{F}_{0}$, we now describe the period map $\mathcal{F}_{0} \rightarrow \mathbb{C} H^{4}$, following [1, (3.11)]. Using the Griffiths residue calculus, one can work out the Hodge numbers of $T$, which turn out to be $h^{3,0}=h^{0,3}=0, h^{2,1}=h^{1,2}=5$. This calculation [1, Lemma 2.6] also gives the refinement of the Hodge decomposition by the eigenspace decomposition

$$
H^{3}(T, \mathbb{C})=H_{\sigma=\omega}^{3}(T, \mathbb{C}) \oplus H_{\sigma=\bar{\omega}}^{3}(T, \mathbb{C})
$$

under $\sigma$, namely $h_{\bar{\omega}}^{2,1}=h_{\omega}^{1,2}=1, h_{\bar{\omega}}^{1,2}=h_{\omega}^{2,1}=4$.

$4^{\mathrm{e}}$ SÉRIE - TOME $43-2010-\mathrm{N}^{\mathrm{o}} 1$ 
Given a framing $[i]$, we may obtain a point of $\mathbb{C} H^{4}$ as follows. Define $i_{*}: H_{\bar{\omega}}^{3}(T, \mathbb{C}) \rightarrow$ $\mathbb{C}^{4,1}$ as the composition

$$
H_{\bar{\omega}}^{3}(T, \mathbb{C}) \cong H^{3}(T, \mathbb{R})=\Lambda(T) \otimes_{\mathcal{E}} \mathbb{C} \underset{i \otimes 1}{\longrightarrow} \Lambda \otimes_{\varepsilon} \mathbb{C}=\mathbb{C}^{4,1}
$$

Here the leftmost isomorphism is the eigenspace projection $H^{3}(T, \mathbb{R}) \rightarrow H_{\bar{\omega}}^{3}(T, \mathbb{C})$, which is an isomorphism of complex vector spaces. This statement has meaning because we have defined $\omega$ to act on $H^{3}(T, \mathbb{Z})$ as $\left(\sigma^{*}\right)^{-1}$. The map is $\mathbb{C}$-linear because the actions of $\bar{\omega} \in \mathbb{C}$ and $\sigma^{*}$ agree on the domain (by the definition of the $\omega$-action as $\left(\sigma^{*}\right)^{-1}$ ) and on the target (since it is the $\bar{\omega}$-eigenspace of $\sigma^{*}$ ). Since the eigenspace projection is obviously $\sigma$-equivariant, it is also $\bar{\omega}$-equivariant, i.e., $\mathbb{C}$-linear.

Our model for complex hyperbolic space $\mathbb{C} H^{4}$ is the set of negative lines in $\mathbb{C}^{4,1}:=\Lambda \otimes_{\mathbb{E}} \mathbb{C}$. It follows from the Riemann bilinear relations that $i_{*}\left(H_{\bar{\omega}}^{2,1}(T, \mathbb{C})\right) \in P\left(\mathbb{C}^{4,1}\right)$ is a negativedefinite line, i.e., a point of $\mathbb{C} H^{4}$. See [1, Lemmas 2.5-2.6], and note that the map called $Z$ there is the eigenspace projection. The period map $g: \mathcal{F}_{0} \rightarrow \mathbb{C} H^{4}$ is then defined by

$$
g(F,[i])=i_{*}\left(H_{\bar{\omega}}^{2,1}(T, \mathbb{C})\right) \in \mathbb{C} H^{4} .
$$

It is holomorphic since the Hodge filtration varies holomorphically [1, (2.16)].

The period map $\mathcal{F}_{0} \rightarrow \mathbb{C} H^{4}$ factors through $\mathcal{M}_{0}^{f}$ because $\mathbb{C} H^{4}$ is a complex ball and bounded holomorphic functions on $G$ are constant. So we may also regard $g$ as a map

$$
g: \mathcal{M}_{0}^{f}=G \backslash \mathcal{F}_{0} \rightarrow \mathbb{C} H^{4} .
$$

This is $P \Gamma$-equivariant, so it descends to another map

$$
g: \mathcal{M}_{0}=G \backslash \mathscr{C}_{0}=(G \times P \Gamma) \backslash \mathcal{F}_{0} \rightarrow P \Gamma \backslash \mathcal{M}_{0}^{f} \rightarrow P \Gamma \backslash \mathbb{C} H^{4},
$$

also called "the period map".

\subsection{The main theorem of $[1]$ in the smooth case}

It turns out that the period map $\mathcal{F}_{0} \rightarrow \mathbb{C} H^{4}$ is not quite surjective. To describe the image, let $\mathscr{H} \subseteq \mathbb{C} H^{4}$ be the union of the orthogonal complements of the norm 1 vectors in $\Lambda$. It turns out that points of $\mathscr{H}$ represent singular cubic surfaces; see Section 10. We will need the following combinatorial result about $\mathcal{H}$, as well as the smooth case of the main theorem of [1].

Lemma 2.3 ([1, (7.29)]). - Any two components of $\mathcal{H}$ that meet are orthogonal along their intersection.

THEOREM 2.4 ([1, Theorem 2.20]). - The periodmap g sends $\mathcal{M}_{0}^{f}=G \backslash \mathcal{F}_{0}$ isomorphically to $\mathbb{C} H^{4}-\mathcal{H}$. In particular, $g$ has everywhere rank 4 on $\mathcal{M}_{0}^{f}$. Moreover, the induced map $\mathcal{M}_{0} \rightarrow P \Gamma \backslash\left(\mathbb{C} H^{4}-\mathcal{H}\right)$ is an isomorphism of complex analytic orbifolds. 


\subsection{Standard model for $H^{2}(S)$; vector spaces over $\mathbb{F}_{3}$}

This material may be skipped until needed in Sections 4 and 8 . We write $L$ for the lattice $\mathbb{Z}^{1,6}$, whose bilinear form is

$$
x \cdot y=x_{0} y_{0}-x_{1} y_{1}-\cdots-x_{6} y_{6},
$$

and write $\eta$ for $(3,-1, \ldots,-1) \in L$. It is standard that $L(S):=H^{2}(S ; \mathbb{Z})$ is isometric to $L$ by an isometry identifying the hyperplane class $\eta(S)$ with $\eta$. (See [25, Thm 23.8]; our expression for $\eta$ corrects a sign error in [1, (3.2)].) Also, the isometry group of $(L, \eta)$ is the Weyl group $W\left(E_{6}\right)$, generated by the reflections in the norm -2 vectors of $L_{0}:=\eta^{\perp}$ (see [25, Theorem 23.9]). We define $L_{0}(S)$ as the corresponding sublattice of $L(S)$, namely the primitive cohomology $\eta(S)^{\perp} \subseteq L(S)$. Our notation $L, \eta, L(S), \eta(S)$ is from [1, (3.2)] and $L_{0}, L_{0}(S)$ is from $[1,(4.8)]$.

In [1, (4.10)] we found a special relationship between $\Lambda(T)$ and $L(S)$ that is needed in Sections 4 and 8 . There is no natural map between them, in either direction. But there is a natural isomorphism between certain $\mathbb{F}_{3}$-vector spaces associated to them. In our explanation we will identify $H^{*}$ and $H_{*}$ by Poincaré duality.

Suppose we have a primitive 2-cycle $c$ on $S$. Since $S \subset T$ and $T$ has no primitive cohomology, there is a 3-chain $d$ in $T$ bounding it. The chain $\sigma_{*}(d)-\sigma_{*}^{-1}(d)$ is a 3-cycle on $T$, whose reduction modulo $\theta$ depends only on $c$ and whose homology class modulo $\theta$ depends only on the homology class of $c$. So we have a natural map $L_{0}(S) \rightarrow \Lambda(T) / \theta \Lambda(T)$. It turns out that the kernel is $3 L_{0}^{\prime}(S)$, where the prime denotes the dual lattice. The result is a natural isomorphism from $V(S):=L_{0}(S) / 3 L_{0}^{\prime}(S)$ to $V(T):=\Lambda(T) / \theta \Lambda(T)$, both fivedimensional vector spaces over $\mathbb{F}_{3}$. We will write $V$ for $\Lambda / \theta \Lambda$.

Also, reducing inner products in $L_{0}^{\prime}(S)$ modulo 3 gives a symmetric bilinear form $q$ on $V(S)$, and similarly, reducing inner products in $\Lambda(T)$ modulo $\theta$ gives one on $V(T)$. We have no special symbol for the latter because it is essentially the same as $q$ :

Lemma $2.5([1,(4.10)])$. - The map $V(S) \rightarrow V(T)$ just defined is an isometry.

A consequence is that the monodromy action of $\pi_{1}\left(\mathscr{C}_{0}, F\right)$ on $V(S)$ is the same as on $V(T)$. Since $P O(V) \cong W\left(E_{6}\right)$, the classical monodromy map

$$
\pi_{1}\left(\mathscr{C}_{0}, F\right) \rightarrow \operatorname{Aut}(L(S), \eta(S)) \cong W\left(E_{6}\right)
$$

can be recovered from our monodromy representation $\pi_{1}\left(\mathscr{C}_{0}, F\right) \rightarrow P \Gamma \rightarrow \mathrm{PO}(V(T))$.

\section{Moduli of smooth real cubic surfaces}

The purpose of this section is to prove those results on moduli of smooth real cubic surfaces which follow more or less automatically from the general results on moduli of complex cubic surfaces that we proved in [1] and summarized in Section 2.

$4^{\text {e }}$ SÉRIE - TOME $43-2010-\mathrm{N}^{\mathrm{o}} 1$ 


\subsection{Anti-involutions and real structures}

We write $\kappa$ for the standard complex conjugation map on $\mathbb{C}^{4}$, and also for the induced map on $\mathscr{C}$ given by

$$
(\kappa . F)(x)=\overline{F\left(\kappa^{-1}(x)\right)}=\overline{F(\kappa x)}
$$

In coordinates this amounts to replacing the coefficients of $F$ by their complex conjugates. This action of $\kappa$ on functions carries holomorphic functions to holomorphic functions (rather than anti-holomorphic ones). Similarly, if $\alpha$ is an anti-holomorphic map of a complex variety $V_{1}$ to another $V_{2}$, then $\overline{\alpha^{*}}: H^{*}\left(V_{2} ; \mathbb{C}\right) \rightarrow H^{*}\left(V_{1} ; \mathbb{C}\right)$ is defined as the usual pullback under $V_{1} \rightarrow V_{2}$ followed by complex conjugation in $H^{*}\left(V_{1} ; \mathbb{C}\right)$. If $V_{1}$ and $V_{2}$ are compact Kähler manifolds, then $\overline{\alpha^{*}}$ is an antilinear map that preserves the Hodge decomposition. Many different complex conjugation maps appear in this paper, so we call a self-map of a complex manifold (resp. complex vector space or $\mathcal{E}$-module) an anti-involution if it is anti-holomorphic (resp. anti-linear) and has order 2. We also use the term real structure for an anti-involution. By the real locus of a real structure we mean the fixed point set of the corresponding anti-involution.

\subsection{Notation}

We write $\mathscr{C}^{\mathbb{R}}, \Delta^{\mathbb{R}}$ and $\mathscr{C}_{0}^{\mathbb{R}}$ for the subsets of the corresponding spaces of $\S 2.1$ whose members have real coefficients. Note that it is possible for the zero locus in $\mathbb{R} P^{3}$ of $F \in \Delta^{\mathbb{R}}$ to be a smooth manifold, for example it might have two complex-conjugate singularities. We write $G^{\mathbb{R}}$ for the group $\operatorname{GL}(4, \mathbb{R})$, which is isomorphic to the group of real points of $G$, and we write $\mathcal{M}_{0}^{\mathbb{R}}$ for the space $G^{\mathbb{R}} \backslash \mathscr{C}_{0}^{\mathbb{R}}$. This is a real-analytic orbifold in the sense that it is locally the quotient of a real analytic manifold by a real analytic action of a finite group. There is a natural map $\mathcal{M}_{0}^{\mathbb{R}} \rightarrow \mathcal{M}_{0}$ which is finite-to-one and generically injective, but not injective (since a cubic surface may have several inequivalent real structures). So $\mathcal{M}_{0}^{\mathbb{R}}$ is not quite the same as the real locus of $\mathcal{M}_{0}$.

\subsection{Framed smooth real cubic surfaces; anti-involutions of $\Lambda$}

We write $\mathcal{F}_{0}^{\mathbb{R}}$ for the preimage of $\mathscr{C}_{0}^{\mathbb{R}}$ in the space $\mathcal{F}_{0}$ of $\S 2.2$. This is not the real locus of any real structure on $\mathcal{F}_{0}$, but rather the union of the real loci of many different real structures. We consider these many different real loci simultaneously because no one of them is distinguished. They are all lifts of $\kappa: \mathscr{C}_{0} \rightarrow \mathscr{C}_{0}$. To develop this idea, let $\mathscr{G}$ denote the set of anti-involutions of $\Lambda$ and let $P \mathscr{G}$ denote the set of their projective equivalence classes. If $(F,[i]) \in \mathcal{F}_{0}^{\mathbb{R}}$ then $\kappa$ acts on $\Lambda(T)$ as an anti-involution, so $\chi=i \circ \kappa^{*} \circ i^{-1}$ lies in $\mathscr{C}$. Because of the ambiguity in the choice of representative $i$ for $[i], \chi$ is not determined by $[i]$; however, its class $[\chi]$ in $P \mathscr{G}$ is well-defined. Clearly $[\chi]$ does not change if $(F,[i])$ varies in a connected component of $\mathcal{F}_{0}^{\mathbb{R}}$. Thus we get a map $\pi_{0}\left(\mathcal{F}_{0}^{\mathbb{R}}\right) \rightarrow P \mathscr{G}$.

The "many different real loci" we referred to are the following subspaces $\mathcal{F}_{0}^{\chi}$ of $\mathcal{F}_{0}$, one for each $[\chi] \in P \mathscr{Q}$ :

$$
\mathcal{F}_{0}^{\chi}=\left\{(F,[i]) \in \mathcal{F}_{0}^{\mathbb{R}}:\left[i \circ \kappa^{*} \circ i^{-1}\right]=[\chi]\right\} .
$$

Here and in many other places we omit the brackets of $[\chi]$ to simplify the notation. The various $\mathcal{F}_{0}^{\chi}$ cover $\mathcal{F}_{0}^{\mathbb{R}}$, because any $(F,[i]) \in \mathcal{F}_{0}^{\mathbb{R}}$ lies in $\mathcal{F}_{0}^{\chi}$ with $\chi=i \circ \kappa^{*} \circ i^{-1}$. We will see 
that each $\mathcal{F}_{0}^{\chi}$ is nonempty. One can check that the lift of $\kappa$ to $\mathcal{F}_{0}$ that fixes $(F,[i])$ has fixedpoint set equal to $\mathcal{F}_{0}^{\chi}$. In fact we give a formula for the action of $\chi$ on $\mathcal{F}_{0}$ in Equation (3.4) below.

Similarly, if $\chi \in P \mathscr{G}$ then we define $H_{\chi}^{4}$ as its fixed-point set in $\mathbb{C} H^{4} \subseteq P\left(\Lambda \otimes_{\mathcal{E}} \mathbb{C}\right)$. The notation reflects the fact that $H_{\chi}^{4}$ is a copy of real hyperbolic 4 -space. Just as for $\mathcal{F}_{0}$, there is no natural choice of lift $\mathbb{C} H^{4} \rightarrow \mathbb{C} H^{4}$ of the action of $\kappa$ on $\mathcal{M}_{0} \cong P \Gamma \backslash \mathbb{C} H^{4}$. So we consider all the $\chi \in P \mathscr{C}$ simultaneously, and the various $H_{\chi}^{4}$ 's are the real loci of the various real structures $\chi$ on $\mathbb{C} H^{4}$.

\subsection{The real period $\operatorname{map} g^{\mathbb{R}}$}

We need the following lemma in order to define the real period map.

Lemma 3.1. - In the notation of $\S 3.3, g\left(\mathcal{F}_{0}^{\chi}\right) \subset H_{\chi}^{4}$.

Proof. - The key is that $\overline{\kappa^{*}}$ is an antilinear map of $H^{3}(T ; \mathbb{C})$ which preserves the Hodge decomposition and each eigenspace of $\sigma$. Therefore it preserves the inclusion $i_{*}\left(H_{\bar{\omega}}^{2,1}(T)\right) \rightarrow \Lambda$. The lemma is a formal consequence of this and the relation $\chi=i \circ \kappa^{*} \circ i^{-1}$.

We define the real period map $g^{\mathbb{R}}: \mathcal{F}_{0}^{\mathbb{R}} \rightarrow \mathbb{C} H^{4} \times P \mathscr{G}$ by

$$
g^{\mathbb{R}}(F,[i])=\left(g(F,[i]),\left[i \circ \kappa^{*} \circ i^{-1}\right]\right) .
$$

The previous lemma asserts that $g(F,[i]) \in H_{\chi}^{4}$, so $g^{\mathbb{R}}(F,[i])$ is a point of $\mathbb{C} H^{4}$ together with an anti-involution fixing it. Therefore $g^{\mathbb{R}}$ can be regarded as a map $\mathcal{F}_{0}^{\mathbb{R}} \rightarrow \bigsqcup_{\chi \in P \mathscr{Q}} H_{\chi}^{4}$. The next lemma shows that $g^{\mathbb{R}}$ descends to a map $G^{\mathbb{R}} \backslash \mathcal{F}_{0}^{\mathbb{R}} \rightarrow \amalg_{\chi \in P \mathscr{G}} H_{\chi}^{4}$.

Lemma 3.2. - The real period map $g^{\mathbb{R}}: \mathcal{F}_{0}^{\mathbb{R}} \rightarrow \amalg_{\chi \in P \mathscr{G}} H_{\chi}^{4}$ is constant on $G^{\mathbb{R}}$-orbits.

Proof. - We must show for $(F,[i]) \in \mathcal{F}_{0}^{\mathbb{R}}$ and $h \in G^{\mathbb{R}}$ that $g^{\mathbb{R}}(h .(F,[i]))=g^{\mathbb{R}}(F,[i])$. We have

$$
\begin{aligned}
g^{\mathbb{R}}(h .(F,[i])) & =g^{\mathbb{R}}\left(h . F,\left[i \circ h^{*}\right]\right) \\
& =\left(g\left(h . F,\left[i \circ h^{*}\right]\right),\left[i \circ h^{*} \circ \kappa^{*} \circ\left(h^{*}\right)^{-1} \circ i^{-1}\right]\right) \\
& =\left(g(h .(F,[i])),\left[i \circ \kappa^{*} \circ i^{-1}\right]\right) \\
& =\left(g(F,[i]),\left[i \circ \kappa^{*} \circ i^{-1}\right]\right) \\
& =g^{\mathbb{R}}(F,[i])
\end{aligned}
$$

Here the first line uses the Definition (2.8) of $G$ 's action on $\mathcal{F}_{0}$, the second the Definition (3.3) of $g^{\mathbb{R}}$, the third the fact that $h$ and $\kappa$ commute, and the fourth the fact that the complex period map $g$ is $G$-invariant. 


\subsection{The main theorem for smooth real surfaces}

We know that $g^{\mathbb{R}}$ cannot map $\mathcal{F}_{0}^{\mathbb{R}}$ onto all of $\amalg_{\chi \in P \mathscr{G}} H_{\chi}^{4}$, because $g\left(\mathcal{F}_{0}\right)$ misses the hyperplane arrangement $\mathscr{H}$. Therefore we define $K_{0}=\amalg_{\chi \in P \mathscr{Q}}\left(H_{\chi}^{4}-\mathscr{H}\right)$. Now we can state the main theorem of this section.

THEOREM 3.3. - The real period map $g^{\mathbb{R}}$ descends to a $P \Gamma$-equivariant real-analytic diffeomorphism $g^{\mathbb{R}}: G^{\mathbb{R}} \backslash \mathcal{F}_{0}^{\mathbb{R}} \rightarrow K_{0}=\coprod_{\chi \in P \mathscr{G}}\left(H_{\chi}^{4}-\mathcal{H}\right)$. Thus $\mathcal{F}_{0}^{\mathbb{R}}$ is a principal $G^{\mathbb{R}}$-bundle over $K_{0}$. Taking the quotient by $P \Gamma$ yields a real-analytic orbifold isomorphism

$$
g^{\mathbb{R}}: \mathcal{M}_{0}^{\mathbb{R}}=\left(P \Gamma \times G^{\mathbb{R}}\right) \backslash \mathcal{F}_{0}^{\mathbb{R}} \rightarrow P \Gamma \backslash K_{0} .
$$

Equivalently, we have an orbifold isomorphism

$$
\mathcal{M}_{0}^{\mathbb{R}} \cong \coprod_{\chi} P \Gamma_{\chi}^{\mathbb{R}} \backslash\left(H_{\chi}-\mathcal{H}\right),
$$

where $\chi$ now ranges over a set of representatives for the set $C \mathscr{G}$ of $P \Gamma$-conjugacy classes of elements of $P \mathscr{Q}$, and $P \Gamma_{\chi}^{\mathbb{R}}$ is the $P \Gamma$-stabilizer of $H_{\chi}^{4}$.

To prove the theorem we extend some of the constructions of Section 2 to include antiholomorphic transformations. These notions will not be needed later in the paper. First, let $\mathrm{GL}(4, \mathbb{C})^{\prime}$ be the group of all linear and antilinear automorphisms of $\mathbb{C}^{4}$. We regard it as also acting on $\mathbb{C}^{5}$, with an element $h$ acting by (2.7) if $h$ is linear and by

$$
h\left(X_{0}, \ldots, X_{3}, Y\right)=\left(h\left(X_{0}, \ldots, X_{3}\right), \bar{Y}\right)
$$

if $h$ is antilinear. If $h$ is linear then it acts on $\mathscr{C}$ as in (2.1), and if $h$ is antilinear then we define

$$
(h . F)\left(X_{0}, \ldots, X_{3}\right)=\overline{F\left(h^{-1}\left(X_{0}, \ldots, X_{3}\right)\right)} .
$$

This is consistent with our Definition (3.1) of the action of $\kappa$.

We let $\mathcal{F}_{0}^{\prime}$ be the space of all pairs $(F,[i])$ where $F \in \mathscr{C}_{0}, i: \Lambda(T) \rightarrow \Lambda$ is either a linear or antilinear isometry, and $[i]$ is its projective equivalence class. $\mathcal{F}_{0}^{\prime}$ is a disjoint union of two copies of $\mathcal{F}_{0}$. Since $\mathcal{F}_{0}$ is connected (Theorem 2.1), $\mathcal{F}_{0}^{\prime}$ has 2 components. Formula (2.8) now defines an action of $\operatorname{GL}(4, \mathbb{C})^{\prime}$ on $\mathcal{F}_{0}^{\prime}$. We also let $\Gamma^{\prime}$ be the group of all linear and antilinear isometries of $\Lambda$, and observe that (2.6) defines an action of it on $\mathcal{F}_{0}^{\prime}$. The antilinear elements in each group exchange the two components of $\mathcal{F}_{0}^{\prime}$. The subgroup $D=\left\{I, \omega I, \omega^{2} I\right\}$ of $\mathrm{GL}(4, \mathbb{C})^{\prime}$ acts trivially, inducing an action of the quotient group, which we call $G^{\prime}$. The scalars in $\Gamma^{\prime}$ also act trivially, inducing an action of the quotient group, which we call $P \Gamma^{\prime}$. Each of $G^{\prime}$ and $P \Gamma^{\prime}$ acts freely on $\mathcal{F}_{0}^{\prime}$, because $G$ and $P \Gamma$ act freely on $\mathcal{F}_{0}$ (Theorem 2.2).

The following two lemmas are generalities about group actions that we will need in the proof of Theorem 3.3. We omit the proof of the first one.

Lemma 3.4. - Let $Y$ be a set and suppose $L$ and $M$ are groups with commuting free actions on it. Suppose $y \in Y$ has images $m \in L \backslash Y$ and $l \in M \backslash Y$. For any $\chi \in L$ preserving $l$, there exists a unique $\hat{\chi} \in M$ such that $(\chi \hat{\chi}) . y=y$. Furthermore, the map $\chi \mapsto \hat{\chi}$ defines an isomorphism from the stabilizer $L_{l}$ of l to the stabilizer $M_{m}$ of $m$.

Lemma 3.5. - If a group $G$ acts freely on a set $X, \phi$ is a transformation of $X$ normalizing $G$, and $Z$ is the centralizer of $\phi$ in $G$, then the natural map $Z \backslash X^{\phi} \rightarrow G \backslash X$ is injective. 
Proof. - If $h \in G$ carries $x \in X^{\phi}$ to $y \in X^{\phi}$ then so does $\phi^{-1} h \phi$, so $\phi^{-1} h \phi=h$ by freeness, so $h \in Z$.

Proof of Theorem 3.3. - First observe that $g^{\mathbb{R}}: G^{\mathbb{R}} \backslash \mathcal{F}_{0}^{\mathbb{R}} \rightarrow K_{0}$ is a local diffeomorphism. This follows immediately from the fact that the rank of $g^{\mathbb{R}}$ is the same as that of $g$, which is 4 everywhere in $G \backslash \mathcal{F}_{0}$ by Theorem 2.4.

To prove surjectivity, suppose $\chi \in P \mathscr{G}$ and $x \in H_{\chi}^{4}-\mathcal{H}$. We must exhibit a point of $\mathcal{F}_{0}^{\mathbb{R}}$ mapping to $(x,[\chi])$ under $g^{\mathbb{R}}$. First, by the surjectivity of the complex period map, there exists $(F,[i]) \in \mathcal{F}_{0}$ with $g(F,[i])=x$. Now we apply Lemma 3.4 with $Y=\mathcal{F}_{0}^{\prime}, y=(F,[i])$, $L=P \Gamma^{\prime}$ and $M=G^{\prime}$. Our choice of $y$ gives

$$
l=x \in \mathbb{C} H^{4}-\mathcal{H}=G^{\prime} \backslash \mathcal{F}_{0}^{\prime}=M \backslash Y .
$$

By hypothesis, $\chi$ is an anti-involution in $P \Gamma^{\prime}$ fixing $x$. By Lemma 3.4 there exists $\hat{\chi} \in G^{\prime}$, of order 2, with $\chi \hat{\chi}$ fixing $(F,[i])$. Since $\chi$ swaps the components of $\mathcal{F}_{0}^{\prime}, \hat{\chi}$ does too, so $\hat{\chi}$ is antiholomorphic. We have constructed a complex cubic surface $\{F=0\}$ preserved by an anti-involution $\hat{\chi} \in G^{\prime}$. Now we will verify that it (or rather a translate of it in $\mathscr{C}_{0}^{\mathbb{R}}$ ) maps to $x$ under $g^{\mathbb{R}}$.

Since $\mathrm{GL}(4, \mathbb{C})^{\prime} \rightarrow G^{\prime}$ has kernel $\mathbb{Z} / 3$, there is an anti-involution $\alpha \in \mathrm{GL}(4, \mathbb{C})^{\prime}$ lying over $\hat{\chi}$. (In fact all 3 elements lying over $\hat{\chi}$ are anti-involutions.) The fact that $\chi \hat{\chi}$ fixes $(F,[i])$ implies $\alpha . F=F$ and $\left[\chi \circ i \circ \alpha^{*}\right]=[i]$, i.e., $\left[i \circ \alpha^{*} \circ i^{-1}\right]=\chi^{-1}=\chi$. Because all real structures on a complex vector space are equivalent, $\alpha$ is conjugate to $\kappa$, that is, there exists $h \in \mathrm{GL}(4, \mathbb{C})$ with $\alpha=h^{-1} \kappa h$. We claim $h .(F,[i])$ lies in $\mathcal{F}_{0}^{\mathbb{R}}$ and maps to $(x, \chi)$ under $g^{\mathbb{R}}$. That it lies in $\mathcal{F}_{0}^{\mathbb{R}}$ is just the claim $h . F \in \mathscr{C}_{0}^{\mathbb{R}}$; here is the verification:

$$
\kappa . h F=h h^{-1} \kappa h . F=h \alpha . F=h . \alpha F=h F .
$$

And finally:

$$
\begin{aligned}
g^{\mathbb{R}}(h \cdot(F,[i])) & =g^{\mathbb{R}}\left(h \cdot F,\left[i \circ h^{*}\right]\right) \\
& =\left(g(h \cdot F),\left[i \circ h^{*} \circ \kappa^{*} \circ\left(h^{*}\right)^{-1} \circ i^{-1}\right]\right) \\
& =\left(g(F),\left[i \circ\left(h^{-1} \circ \kappa \circ h\right)^{*} \circ i^{-1}\right]\right) \\
& =\left(x,\left[i \circ \alpha^{*} \circ i^{-1}\right]\right) \\
& =(x, \chi) .
\end{aligned}
$$

This finishes the proof of surjectivity.

To prove injectivity it suffices to show that $g^{\mathbb{R}}: G^{\mathbb{R}} \backslash \mathcal{F}_{0}^{\chi} \rightarrow G \backslash \mathcal{F}_{0}=\mathbb{C} H^{4}-\mathcal{H}_{\text {is injective }}$ for each $\chi \in P \mathscr{G}$. This also follows from a general principle, best expressed by regarding $\mathcal{F}_{0}^{\chi}$ as the fixed-point set of $\chi$ in $\mathcal{F}_{0}$. We have formulated an action of $P \Gamma^{\prime}$ on $\mathcal{F}_{0}^{\prime}$, but we can regard it as acting on $\mathcal{F}_{0}$ by identifying $\mathcal{F}_{0}$ with $\langle\kappa\rangle \backslash \mathcal{F}_{0}^{\prime}$. The subgroup $P \Gamma$ acts by (2.6) as before, but an anti-linear $\gamma \in P \Gamma^{\prime}$ now acts by

$$
\gamma \cdot(F,[i])=\left(\kappa . F,\left[\gamma \circ i \circ \kappa^{*}\right]\right) .
$$

It follows from these definitions that $\mathcal{F}_{0}^{\chi}$ is the fixed-point set of $\chi$. We apply Lemma 3.5 with $X=\mathcal{F}_{0}, G=G$ and $\phi=\chi$; then $X^{\phi}=\mathcal{F}_{0}^{\chi}$ and $Z=G^{\mathbb{R}}$. The conclusion is that $G^{\mathbb{R}} \backslash \mathcal{F}_{0}^{\chi} \rightarrow G \backslash \mathcal{F}_{0}=\mathbb{C} H^{4}-\mathscr{H}$ is injective. This concludes the proof of the first statement of the theorem. The remaining statements follow. 


\section{The five families of real cubics}

Theorem 3.3 described $\mathcal{M}_{0}^{\mathbb{R}}$ in terms of the $H_{\chi}^{4}$, where $[\chi]$ varies over a complete set of representatives of $C \mathscr{G}$, the set of $P \Gamma$-conjugacy classes in the set $P \mathscr{C}$ of projective equivalence classes of anti-involutions of $\Lambda$. In this section we find such a set of representatives. That is, we classify the $\chi$ up to conjugacy by $\Gamma$; there are exactly 10 classes, and we give a recognition principle which allows one to easily compute which class contains a given anti-involution. In fact there are only five classes up to sign, so $C \mathscr{G}$ has 5 elements, and there are 5 orbits of $H_{\chi}^{4}$ 's under $P \Gamma$. Unlike in the rest of the paper, in this section we will be careful to distinguish between an anti-involution $\chi$ of $\Lambda$ and its projective equivalence class $[\chi]$.

\subsection{Classification of anti-involutions of $\Lambda$}

Using the coordinate system (2.5), we define the following five anti-involutions of $\Lambda$ :

$$
\begin{aligned}
& \chi_{0}:\left(x_{0}, x_{1}, x_{2}, x_{3}, x_{4}\right) \mapsto\left(\bar{x}_{0}, \quad \bar{x}_{1}, \quad \bar{x}_{2}, \quad \bar{x}_{3}, \bar{x}_{4}\right) \\
& \chi_{1}:\left(x_{0}, x_{1}, x_{2}, x_{3}, x_{4}\right) \mapsto\left(\bar{x}_{0}, \quad \bar{x}_{1}, \quad \bar{x}_{2}, \quad \bar{x}_{3},-\bar{x}_{4}\right) \\
& \chi_{2}:\left(x_{0}, x_{1}, x_{2}, x_{3}, x_{4}\right) \mapsto\left(\bar{x}_{0}, \quad \bar{x}_{1}, \quad \bar{x}_{2},-\bar{x}_{3},-\bar{x}_{4}\right) \\
& \chi_{3}:\left(x_{0}, x_{1}, x_{2}, x_{3}, x_{4}\right) \mapsto\left(\bar{x}_{0}, \quad \bar{x}_{1},-\bar{x}_{2},-\bar{x}_{3},-\bar{x}_{4}\right) \\
& \chi_{4}:\left(x_{0}, x_{1}, x_{2}, x_{3}, x_{4}\right) \mapsto\left(\bar{x}_{0},-\bar{x}_{1},-\bar{x}_{2},-\bar{x}_{3},-\bar{x}_{4}\right) .
\end{aligned}
$$

The subscript indicates how many of the coordinates are replaced by the negatives of their complex conjugates rather than just their conjugates.

In order to distinguish their conjugacy classes we will use the 5 -dimensional vector space $V=\Lambda / \theta \Lambda$ over the field $\mathbb{F}_{3}=\delta / \theta \mathcal{E}$, and its quadratic form $q$, the reduction of the Hermitian form (2.5). These were defined in $\S 2.6$. The dimensions of $\chi$ 's eigenspaces and the determinants of $q$ 's restrictions to them are conjugacy invariants of $\chi$ (the determinants lie in $\left.\mathbb{F}_{3}^{*} /\left(\mathbb{F}_{3}^{*}\right)^{2}=\{ \pm 1\}\right)$. We use the abbreviation negated space for the $(-1)$-eigenspace of $\chi$.

Theorem 4.1. - An anti-involution of $\Lambda$ is $\Gamma$-conjugate to exactly one of the $\pm \chi_{j}$. Two anti-involutions of $\Lambda$ are conjugate if and only if the restrictions of $q$ to the two fixed spaces in $V$ (or to the two negated spaces) have the same dimension and determinant.

CAUTION. - The obvious analogue of the theorem fails for some other $\mathcal{E}^{n, 1}$, for example $n=3$. See Remark 6 at the end of [4].

Proof. - It is classical that $P \mathscr{C}_{0}^{\mathbb{R}}$ has 5 connected components [34, §24]. Because -1 lies in the identity component of $G^{\mathbb{R}}$, it follows that $\mathscr{C}_{0}^{\mathbb{R}}$ itself has 5 components, and thence that $\mathcal{M}_{0}^{\mathbb{R}}$ has at most 5 components. The surjectivity part of Theorem 3.3 implies that for every $\chi \in \mathscr{Q}$ there exists $F \in \mathscr{C}_{0}^{\mathbb{R}}$ such that $\left(\Lambda(T),\left[\kappa^{*}\right]\right) \cong(\Lambda,[\chi])$. Therefore the number of components of $\mathcal{M}_{0}^{\mathbb{R}}$ is at least the cardinality of $C \mathscr{G}$, so $|C \mathscr{G}| \leq 5$. Also, the elements of $[\chi]$ are $\chi \cdot(-\omega)^{i}, i=0, \ldots, 5$, and these fall into at most two conjugacy classes (proof: conjugate by scalars). Therefore there are at most 10 classes of anti-involutions of $\Lambda$.

Now we exhibit 10 distinct classes. It is easy to check that $\chi_{j}$ has negated (resp. fixed) space of dimension $j$ (resp. $5-j$ ) and the restriction of $q$ to it has determinant +1 (resp. $-1)$. For $-\chi_{j}$, the negated and fixed spaces are reversed. Therefore $\pm \chi_{0}, \ldots, \pm \chi_{4}$ all lie in 
distinct conjugacy classes. Since we have exhibited 10 classes, they must be a complete set of representatives, justifying the first part of the theorem. In distinguishing them, we also proved the second part.

Let $H_{j}^{4}$ be the fixed-point set of $\chi_{j}$ in $\mathbb{C} H^{4}$, and let $P \Gamma_{j}^{\mathbb{R}}$ be the stabilizer of $H_{j}^{4}$ in $P \Gamma$. We have the following improvement on Theorem 3.3.

Corollary 4.2. - The set $C \mathscr{Q}$ has cardinality 5 and is represented by $\chi_{0} \ldots, \chi_{4}$ of (4.1). We have an isomorphism $\mathcal{M}_{0}^{\mathbb{R}}=\coprod_{j=0}^{4} P \Gamma_{j}^{\mathbb{R}} \backslash\left(H_{j}^{4}-\mathcal{H}\right)$ of real analytic orbifolds. For each $j$, $P \Gamma_{j}^{\mathbb{R}} \backslash\left(H_{j}^{4}-\mathcal{H}\right)$ is connected.

\subsection{The classical labeling of the five components}

The classical labeling of the 5 types of real cubic surfaces was in terms of the topology of the real locus of $S$, or the action of complex conjugation $\kappa$ on the 27 lines of $S$, or the action of $\kappa$ on $H^{2}(S)$. We will develop enough of this to establish the correspondence between the 5 types of surfaces and our $P \Gamma_{j}^{\mathbb{R}} \backslash\left(H_{j}^{4}-\mathcal{H}\right)$.

Recall from $\S 2.6$ the lattice $L(S)=H^{2}(S ; \mathbb{Z})$, the hyperplane class $\eta(S)$, the primitive cohomology $L_{0}(S)$, and their "standard models" $L, \eta, L_{0}$. As stated there, the isometries of $L(S)$ preserving $\eta(S)$ form a copy of the Weyl group $W\left(E_{6}\right)=\operatorname{Aut}(L, \eta)$, which is generated by the reflections in the roots (norm -2 vectors) of $L_{0}$.

Since $\kappa$ is antiholomorphic, it negates $\eta(S)$ and hence acts on $L(S)$ by the product of $-I$ and some element $g$ of Aut $(L(S), \eta(S))$ of order 1 or 2 . Therefore, to classify the possible actions of $\kappa$ on $L(S)$ we will enumerate the involutions of $W\left(E_{6}\right)$ up to conjugacy. According to [9, p. 27] or [25, Table 1], there are exactly four conjugacy classes of involutions. Each class may be constructed as the product of the reflections in $1 \leq j \leq 4$ mutually orthogonal roots. To make this explicit we choose four distinct commuting reflections $R_{1}, \ldots, R_{4}$ in $W\left(E_{6}\right)$.

We write $\mathscr{C}_{0,0}^{\mathbb{R}}, \ldots, \mathscr{C}_{0,4}^{\mathbb{R}}$ for the set of those $F \in \mathscr{C}_{0}^{\mathbb{R}}$ for which $\left(L(S), \eta(S), \kappa^{*}\right)$ is equivalent to $(L, \eta,-g)$ for $g=I, R_{1}, R_{1} R_{2}, R_{1} R_{2} R_{3}, R_{1} R_{2} R_{3} R_{4}$. The $j$ in $\mathscr{C}_{0, j}^{\mathbb{R}}$ is the

number of $R$ 's involved. By the previous paragraph, the $\mathscr{C}_{0, j}^{\mathbb{R}}$ are disjoint and cover $\mathscr{C}_{0}^{\mathbb{R}}$. We will write $\mathcal{M}_{0, j}^{\mathbb{R}}$ for $G^{\mathbb{R}} \backslash \mathscr{C}_{0, j}^{\mathbb{R}}$.

Now we relate the $\kappa$-action on $L(S)$ to the configuration of lines. In the terminology of [34, §23], a line is called real if it is preserved by $\kappa$, and a non-real line is said to be of the first (resp. second) kind if it meets (resp. does not meet) its complex conjugate. The terminology becomes a little easier to remember if one thinks of a real line as being a line of the 0th kind. The lines define 27 elements of $L(S)$, which are exactly the 27 vectors of norm -1 that have inner product 1 with $\eta(S),[25, \S 23]$. Two lines meet (resp. do not meet) if the corresponding vectors have inner product 1 (resp. 0). So which lines of $S$ are real or nonreal of the first or second kind can be determined by studying the action of $\kappa$ on $L(S)$. The number of lines of the various types depends only on the isometry class of $\left(L(S), \eta(S), \kappa^{*}\right)$, with the results given in the first five columns of table 4.1. This allows us to identify our $\mathscr{C}_{0, j}^{\mathbb{R}}$ with the classically defined families. For example, Segre $[34, \S 23]$ names the families $F_{1}, \ldots, F_{5}$; his $F_{j+1}$ corresponds to our $\mathscr{C}_{0, j}^{\mathbb{R}}$. 


\begin{tabular}{cccccccc} 
& class of & \multicolumn{3}{c}{ non-real of } & fixed & fixed & class of \\
action & real & $1^{\text {st }}$ & $2^{\text {nd }}$ & space & space & action \\
family & on $L(S)$ & lines & kind & kind & in $V(S)$ & in $V(T)$ & on $\Lambda(T)$ \\
\hline $\mathscr{C}_{0,0}^{\mathbb{R}}$ & $-I$ & 27 & 0 & 0 & {[]} & {$[++++-]$} & $\chi_{0}$ \\
$\mathscr{C}_{0,1}^{\mathbb{R}}$ & $-R_{1}$ & 15 & 0 & 12 & {$[+]$} & {$[+++-]$} & $\chi_{1}$ \\
$\mathscr{C}_{0,2}^{\mathbb{R}}$ & $-R_{1} R_{2}$ & 7 & 4 & 16 & {$[++]$} & {$[++-]$} & $\chi_{2}$ \\
$\mathscr{C}_{0,3}^{\mathbb{R}}$ & $-R_{1} R_{2} R_{3}$ & 3 & 12 & 12 & {$[+++]$} & {$[+-]$} & $\chi_{3}$ \\
$\mathscr{C}_{0,4}^{\mathbb{R}}$ & $-R_{1} R_{2} R_{3} R_{4}$ & 3 & 24 & 0 & {$[++++]$} & {$[-]$} & $\chi_{4}$
\end{tabular}

TABLE 4.1. Action of complex conjugation on various objects associated to $F \in \mathscr{C}_{0, j}^{\mathbb{R}}$. The $6^{\text {th }}$ and $7^{\text {th }}$ columns indicate diagonalized $\mathbb{F}_{3}$-quadratic forms with \pm 1 's on the diagonal.

\subsection{Relation between our anti-involutions and the classical labeling}

In Corollary 4.2 and $\S 4.2$ we give two labelings for the components of $\mathcal{M}_{0}^{\mathbb{R}}$, namely

$$
\coprod_{j=0}^{4} \mathcal{M}_{0, j}^{\mathbb{R}}=\mathcal{M}_{0}^{\mathbb{R}} \cong \coprod_{j=0}^{4}\left(G^{\mathbb{R}} \times P \Gamma_{j}^{\mathbb{R}}\right) \backslash \mathcal{F}_{0}^{\chi_{j}} \cong \coprod_{j=0}^{4} P \Gamma_{j}^{\mathbb{R}} \backslash\left(H_{j}^{4}-\mathcal{H}\right) \text {. }
$$

Our next goal is Corollary 4.5, which shows that the labelings correspond in the obvious way. We defined the spaces $\mathscr{C}_{0, j}^{\mathbb{R}}$ and $\mathcal{M}_{0, j}^{\mathbb{R}}$ in terms of the action of complex conjugation $\kappa$ on $L(S)$, and we defined the spaces $\mathcal{F}_{0}^{\chi_{j}}$ in terms of $\kappa$ 's action on $\Lambda(T)$. To relate them, we consider the action of $\kappa$ on the 5-dimensional quadratic $\mathbb{F}_{3}$-vector spaces $V(S), V(T)$ defined in terms of $L(S)$ and $\Lambda(T)$ in $\$ 2.6$.

Lemma 4.3. - Let $F \in \mathscr{C}_{0}^{\mathbb{R}}$ and denote the actions of $\kappa$ on $V(S)$ and $V(T)$ by $\hat{\kappa}$. Then $(V(S), \hat{\kappa})$ and $(V(T),-\hat{\kappa})$ are isomorphic as quadratic spaces equipped with isometries.

Proof. - By Lemma 2.5 there is a natural isometry $V(S) \rightarrow V(T)$, which we will denote by $A$. If $a \in V(S)$ then $A(a)$ is defined by lifting $a$ to some $c \in L_{0}(S)$ and then applying the construction in $\$ 2.6$. The result is the reduction modulo $\theta$ of the homology class of $\sigma_{*}(d)-\sigma_{*}^{-1}(d)$ for some 3-chain $d$ in $T$. (The asterisk in the subscript comes from our identification of homology and cohomology in \$2.6.) From this and the fact that $\kappa \sigma=\sigma^{-1} \kappa$ it follows that $A \hat{\kappa}=-\hat{\kappa} A$. Therefore $A$ is an isometry between the pairs $(V(S), \hat{\kappa})$ and $(V(T),-\hat{\kappa})$.

Lemma 4.4. - Suppose $F \in \mathscr{C}_{0, j}^{\mathbb{R}}$. Then the isometry classes of the fixed spaces for $\kappa$ in $V(S)$ and $V(T)$ are given by the $6^{\text {th }}$ and $7^{\text {th }}$ columns of table 4.1, and $\left(\Lambda(T), \kappa^{*}\right)$ is isometric to $\left(\Lambda, \chi_{j}\right)$ as indicated in the last column.

Proof. - Since the conjugacy class of the action of $\kappa$ on $L_{0}(S)$ is known, it is easy to compute the fixed space in $V(S)$. It is just the span of the images of the roots corresponding to $R_{1}, \ldots, R_{j}$. This space has dimension $j$, and its determinant is +1 because the roots have norm $-2 \equiv 1(\bmod 3)$. This justifies the $6^{\text {th }}$ column. Lemma 4.3 shows that the fixed space in $V(T)$ is isometric to the negated space in $V(S)$, justifying the $7^{\text {th }}$ column. The last claim follows from Theorem 4.1. 
COROLlaRY 4.5. - We have $\mathcal{M}_{0, j}^{\mathbb{R}} \cong P \Gamma_{j}^{\mathbb{R}} \backslash\left(H_{j}^{4}-\mathcal{H}\right)$ for $j=0, \ldots, 4$.

\section{The stabilizers of the $H^{4}$ 's}

In this section we continue to make Theorem 3.3 more explicit; we know that $\mathcal{H}_{0}^{\mathbb{R}}=\coprod_{j=0}^{4} P \Gamma_{j}^{\mathbb{R}} \backslash\left(H_{j}^{4}-\mathcal{H}\right)$, and now we will describe the $P \Gamma_{j}^{\mathbb{R}}$. We give two descriptions, one arithmetic and one in the language of Coxeter groups. The arithmetic description is easy:

THEOREM 5.1. - $P \Gamma_{j}^{\mathbb{R}} \cong \mathrm{PO}\left(\Psi_{j}\right)$, where $\Psi_{j}$ is the quadratic form on $\mathbb{Z}^{5}$ given by

$$
\begin{aligned}
& \Psi_{0}\left(y_{0}, \ldots, y_{4}\right)=-y_{0}^{2}+y_{1}^{2}+y_{2}^{2}+y_{3}^{2}+y_{4}^{2} \\
& \Psi_{1}\left(y_{0}, \ldots, y_{4}\right)=-y_{0}^{2}+y_{1}^{2}+y_{2}^{2}+y_{3}^{2}+3 y_{4}^{2} \\
& \Psi_{2}\left(y_{0}, \ldots, y_{4}\right)=-y_{0}^{2}+y_{1}^{2}+y_{2}^{2}+3 y_{3}^{2}+3 y_{4}^{2} \\
& \Psi_{3}\left(y_{0}, \ldots, y_{4}\right)=-y_{0}^{2}+y_{1}^{2}+3 y_{2}^{2}+3 y_{3}^{2}+3 y_{4}^{2} \\
& \Psi_{4}\left(y_{0}, \ldots, y_{4}\right)=-y_{0}^{2}+3 y_{1}^{2}+3 y_{2}^{2}+3 y_{3}^{2}+3 y_{4}^{2} .
\end{aligned}
$$

The mnemonic is that $j$ of the coefficients of $\Psi_{j}$ are 3 rather than 1 . To prove the theorem, write $\Lambda_{j}:=\Lambda^{\chi_{j}}$ for the $\mathbb{Z}$-lattice of $\chi_{j}$-invariant vectors in $\Lambda$, so $\Lambda_{j}=\mathbb{Z}^{5-j} \oplus \theta \mathbb{Z}^{j} \subseteq \mathcal{E}^{5}$. The theorem now follows from this lemma:

Lemma 5.2. - For each $j$, every isometry of the $\mathbb{Z}$-lattice $\Lambda_{j}$ is induced by an isometry of $\Lambda$.

Proof. - One can check that the $\mathbb{Z}$-lattice $L:=\Lambda_{j} \cap \theta \Lambda$ can be described in terms of $\Lambda_{j}$ alone as $L=3\left(\Lambda_{j}\right)^{\prime}$, where the prime denotes the dual lattice. Therefore every isometry of $\Lambda_{j}$ preserves the $\mathcal{E}$-span of $\Lambda_{j}$ and $\frac{1}{\theta} L$, which in each case is exactly $\Lambda$.

\subsection{Reflection groups and Coxeter diagrams}

Now we describe the $P \Gamma_{j}^{\mathbb{R}}$ more geometrically; this is interesting in its own right, and also necessary for when we allow our cubic surfaces to have singularities (Section 11). Our description relies on the good fortune that the subgroup $W_{j}$ generated by reflections has index 1 or 2 in each case. The $W_{j}$ are Coxeter groups, described in figures 1.1 and 5.1 using an extension of the usual conventions for Coxeter diagrams, which we now explain. For background on Coxeter groups in this context, see [37].

Namely, the mirrors (fixed-point sets) of the reflections in $W_{j}$ chop $H_{j}^{4}$ into components, which $W_{j}$ permutes freely and transitively. The closure of any one of these components is called a Weyl chamber; we fix one and call it $C_{j}$. Then $W_{j}$ is generated by the reflections across the facets of $C_{j}$, and $C_{j}$ is a fundamental domain in the strong sense that any point of $H_{j}^{4}$ is $W_{j}$-equivalent to a unique point of $C_{j}$. We describe $W_{j}$ by drawing its Coxeter diagram: its vertices ("nodes") correspond to the facets of $C_{j}$, which are joined by edges 
("bonds") that are decorated according to how facets meet each other, using the following scheme:

$$
\begin{aligned}
& \text { no bond } \bigcirc \quad \bigcirc \Longleftrightarrow \text { they meet orthogonally; } \\
& \text { a single bond } \bigcirc-\circ \Longleftrightarrow \text { their interior angle is } \pi / 3 \text {; } \\
& \text { a double bond } \bigcirc=0 \Longleftrightarrow \text { their interior angle is } \pi / 4 \text {; } \\
& \text { a triple bond } \rightleftharpoons 0 \Longleftrightarrow \text { their interior angle is } \pi / 6 \text {; } \\
& \text { a strong bond } \bigcirc-\bigcirc \text { they are parallel; } \\
& \text { a weak bond } \bigcirc----\circ \Longleftrightarrow \text { they are ultraparallel. }
\end{aligned}
$$

Parallel walls are those that do not meet in hyperbolic space but do meet at the sphere at infinity. Ultraparallel walls are those that do not meet even at infinity.

Note that the diagram for $W_{j}$ admits a symmetry for $j=1$ or 2 ; this represents an isometry of $C_{j}$. We now state the main theorem of this section.

THeOREM 5.3. $-P \Gamma_{j}^{\mathbb{R}}$ is the semidirect product of its reflection subgroup $W_{j}$, given in Fig. 1.1 and in more detail in Fig. 5.1, by the group of diagram automorphisms, which is $\mathbb{Z} / 2$ if $j=1$ or 2 and trivial otherwise.

The rest of the section is devoted to the proof. For the most part the argument is uniform in $j$, so we will write $H$ for $H_{j}^{4}=H_{\chi_{j}}^{4}, W$ for $W_{j}, \chi$ for $\chi_{j}$ and $C$ for $C_{j}$. We will write $\Lambda^{\chi}$ for $\Lambda_{j}=\Lambda^{\chi_{j}}$. We call $r \in \Lambda^{\chi}$ a root of $\Lambda^{\chi}$ if it is primitive, has positive norm, and the reflection in it,

$$
x \mapsto x-2 \frac{x \cdot r}{r^{2}} r,
$$

preserves $\Lambda^{\chi}$. It is easy to say what the roots are:

Lemma 5.4. - Suppose $r \in \Lambda^{\chi}$ is primitive in $\Lambda^{\chi}$ and has positive norm. Then $r$ is a root of $\Lambda^{\chi}$ if and only if either $r^{2} \in\{1,2\}$ or else $r^{2} \in\{3,6\}$ and $r \in 3\left(\Lambda^{\chi}\right)^{\prime}$, where the prime denotes the dual lattice.

REMARK. - Norm 3 and 6 roots are really just norm 1 and 2 roots of $\Lambda$ in disguise. They are primitive in $\Lambda^{\chi}$ but divisible by $\theta$ in $\Lambda$, and occur when $\chi$ negates rather than preserves a norm 1 or 2 vector of $\Lambda$.

Proof. - Because $\Lambda^{\chi}$ is 3-elementary (the quotient by its dual lattice is an elementary abelian 3-group), any primitive $r \in \Lambda^{\chi}$ has $3 \mathbb{Z} \subseteq r \cdot \Lambda^{\chi}$. If $r$ is also a root then $r \cdot \Lambda^{\chi} \subseteq \frac{1}{2} r^{2} \mathbb{Z}$, so $r^{2} \mid 6$. It is obvious that every norm 1 or 2 vector is a root, and it is easy to see that a norm 3 or 6 vector is a root if and only if it lies in $3\left(\Lambda^{\chi}\right)^{\prime}$. 


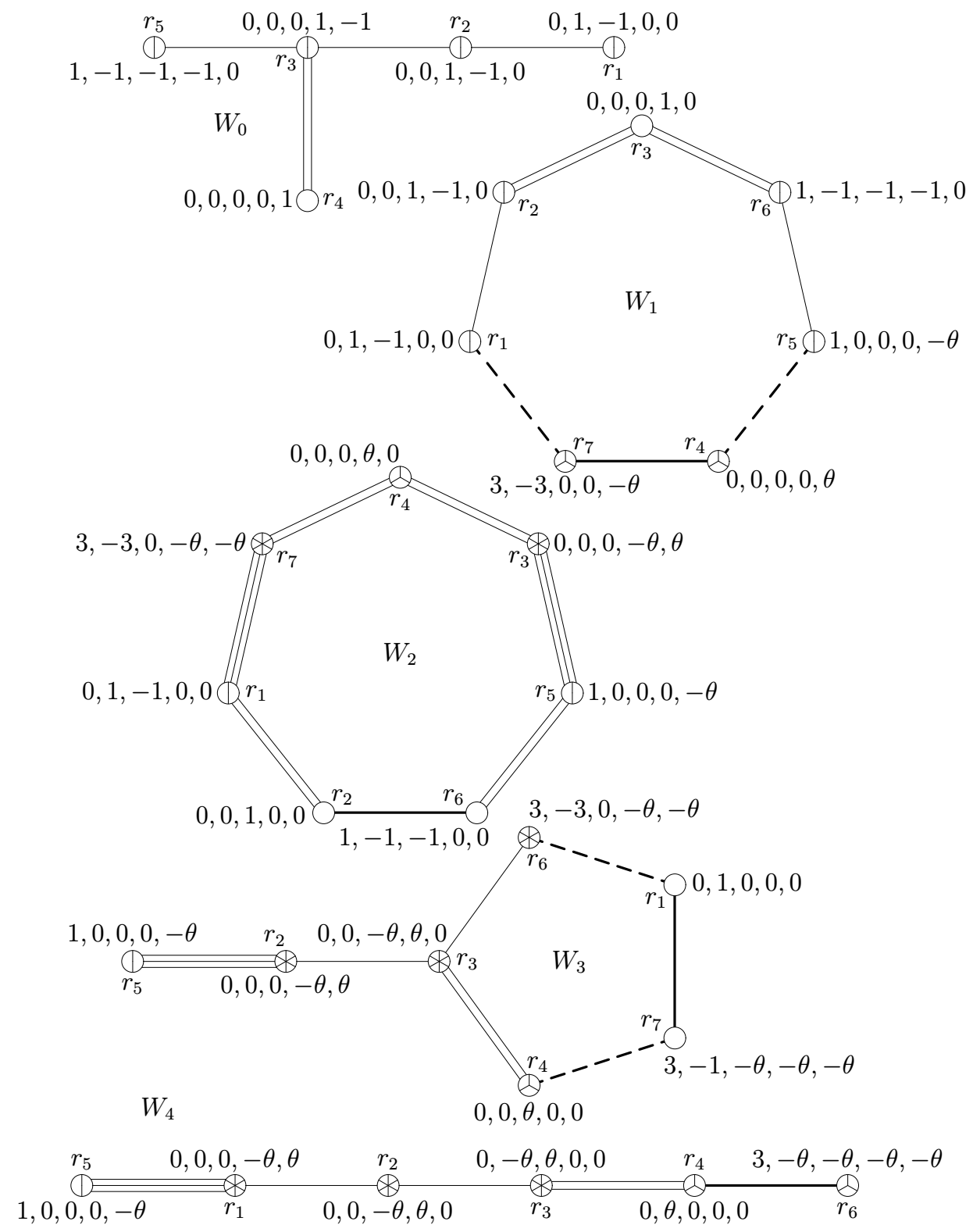

FigURE 5.1. Simple roots for the $W_{j}$.

\subsection{Simple roots and Vinberg's algorithm}

Given some roots $r_{1}, \ldots, r_{n}$ of $\Lambda^{\chi}$ whose inner products are non-positive, their polyhedron is defined to be a particular one of the regions bounded by the hyperplanes $r_{i}^{\perp}$, namely the image in $H$ of

$$
\left\{v \in \Lambda^{\chi} \otimes \mathbb{R} \mid v^{2}<0 \text { and } v \cdot r_{i} \leq 0 \text { for } i=1, \ldots, n\right\} .
$$

$4^{\mathrm{e}}$ SÉRIE - TOME $43-2010-\mathrm{N}^{\mathrm{o}} 1$ 
A set of simple roots for $W$ is a set of roots of $\Lambda^{\chi}$ whose pairwise inner products are nonpositive and whose polyhedron is a Weyl chamber $C$. Vinberg's algorithm [38] seeks a set of simple roots for $W$. We briefly outline how we use this algorithm.

First one chooses a vector $k$ (the "controlling vector") representing a point $p$ of $H$. We choose $k=(1,0,0,0,0)$, which conveniently lies in all the $\Lambda^{\chi_{j}}$. Second, one considers the finite subgroup of $W$ generated by the reflections in $W$ that fix $p$. These are the reflections in the roots of $\Lambda^{\chi}$ that are orthogonal to $k$.

In each case it is easy to enumerate these roots, recognize the finite Weyl group generated by their reflections, and extract a set of simple roots for this finite group. For example, for $j=2$ the roots are $(0, \pm 1,0,0,0),(0,0, \pm 1,0,0),(0,0,0, \pm \theta, 0),(0,0,0,0, \pm \theta)$, $(0, \pm 1, \pm 1,0,0)$ and $(0,0,0, \pm \theta, \pm \theta)$, the finite Weyl group has type $B_{2} \times B_{2}$, and a set of simple roots is $(0,1,-1,0,0),(0,0,1,0,0),(0,0,0, \bar{\theta}, \theta)$ and $(0,0,0, \theta, 0)$. In each of the 5 cases we called the simple roots $r_{1}, \ldots, r_{4}$, and they can be found in Fig. 5.1.

In that figure, a node indicated by $\bigcirc$ (resp. $(1, \otimes, \otimes$ ) represents a root of norm 1 (resp. 2, 3,6). The mnemonic is that the norm of the root is the number of white regions in the symbol. Nodes are joined according to (5.1).

Next, one orders the mirrors of $W$ that miss $p$ according to their "priority", where the priority is any decreasing function of the distance to $p$. The iterative step in Vinberg's algorithm is to consider all roots of a given priority $p$, and suppose that previous batches have enumerated all simple roots of higher priority. Batch 0 has already been defined. We discard those roots of priority $p$ that have positive inner product with some simple root of a previous batch. Those that remain are simple roots and form the current batch. If the polyhedron $P$ defined by our newly-enlarged set of simple roots has finite volume then the algorithm terminates. Otherwise, we proceed to the next batch. The finite-volume condition can be checked using a criterion of Vinberg [39, p. 22] on the simple roots. There is no guarantee that the algorithm will terminate, but if it does then the roots obtained (the union of all the batches) form a set of simple roots for $W$. The algorithm terminates in our five cases, with simple roots given in Fig. 5.1.

Now we can finish the proof of Theorem 5.3, which describes $P \Gamma_{j}^{\mathbb{R}}$ as the semidirect product of $W_{j}$ by its group of diagram automorphisms. $W_{j}$ is obviously a normal subgroup of $P \Gamma_{j}^{\mathbb{R}}$. It follows that $P \Gamma_{j}^{\mathbb{R}}$ is the semidirect product of $W_{j}$ by the subgroup of $P \Gamma_{j}^{\mathbb{R}}$ that carries $C_{j}$ to itself. In cases $j=0,3$ and $4, C_{j}$ has no symmetry, so $P \Gamma_{j}^{\mathbb{R}}=W_{j}$ as claimed. In the remaining cases all we have to do is check is that the nontrivial diagram automorphism $\gamma$ lies in $P \Gamma_{j}^{\mathbb{R}}$. In each case, the simple roots span $\Lambda_{j}$, and $\gamma$ preserves their norms and inner products. So $\gamma \in P \Gamma_{j}^{\mathbb{R}}$ by Lemma 5.2.

\section{The discriminant in the real moduli space}

Theorem 3.3 identifies the moduli space $G^{\mathbb{R}} \backslash \mathcal{F}_{0}^{\mathbb{R}}$ of smooth framed real cubics with the incomplete hyperbolic manifold $K_{0}$, which is the disjoint union of the $H_{\chi}^{4}-\mathscr{H}$. Here $\chi$ varies over the set $P \mathscr{G}$ of projective classes of anti-involutions of $\Lambda$, as in $\S 3.3$, and $\mathcal{H}$ is the locus in $\mathbb{C} H^{4}$ representing the singular cubic surfaces, defined in $\S 2.5$. For a concrete understanding of $K_{0}$ we need to understand how $\mathcal{H}$ meets the various $H_{\chi}^{4}$ 's. Since $\mathcal{H}$ is the union of the 
orthogonal complements $r^{\perp}$ of the norm 1 vectors $r$ of $\Lambda$, we will study how such an $r^{\perp}$ can meet one of the $H_{\chi}^{4}$ 's. We will call a component $r^{\perp}$ of $\mathcal{H}$ a discriminant mirror.

If $\chi$ is an anti-involution of $\Lambda$, then one way $H_{\chi}^{4}$ can meet $r^{\perp}$ is if $\chi(r)= \pm r$; then $H_{\chi}^{4} \cap r^{\perp}$ is a copy of $H^{3}$. But a more complicated intersection can occur; to describe it we need the idea of a $G_{2}$ root system in $\Lambda^{\chi}$. As in Section 5, a root of $\Lambda^{\chi}$ means a norm 1 or 2 vector of $\Lambda^{\chi}$, or a norm 3 or 6 vector of $\Lambda^{\chi}$ that is divisible in $\Lambda$ by $\theta$. By a $G_{2}$ root system in $\Lambda^{\chi}$ we mean a set of six roots of norm 2 and six roots of norm 6 , all lying in a two-dimensional sublattice of $\Lambda^{\chi}$. Such a set of vectors automatically forms a copy of what is commonly known as the $G_{2}$ root system. The reason these root systems are important is that each $G_{2}$ root system $R$ in $\Lambda^{\chi}$ determines an isometric copy of $\mathcal{E}^{2}$ in $\Lambda$, and hence two discriminant mirrors. The $\mathcal{E}^{2}$ is just $\Lambda \cap\left(\langle R\rangle \otimes_{\mathbb{Z}} \mathbb{C}\right)$. To see this, introduce coordinates on the complex span of $R$, in which $R$ consists of the vectors obtained by permuting the coordinates of $(1,-1,0)$ and $\pm(2,-1,-1)$ in the space

$$
\mathbb{C}^{2}=\left\{(x, y, z) \in \mathbb{C}^{3}: x+y+z=0\right\},
$$

with the usual metric. Since $\Lambda$ contains $\frac{1}{\theta}$ times the norm 6 roots, it also contains

$$
\begin{aligned}
& r_{1}=\frac{1}{\theta}(2,-1,-1)+\omega(1,-1,0)=-\frac{1}{\theta}(\omega, \bar{\omega}, 1) \text { and } \\
& r_{2}=-\frac{1}{\theta}(2,-1,-1)+\bar{\omega}(1,-1,0)=\frac{1}{\theta}(\bar{\omega}, \omega, 1) .
\end{aligned}
$$

These have norm 1 and are orthogonal, so they span a copy of $\mathcal{E}^{2}$. Observe also that $\chi$ exchanges the $r_{i}$, and that each of the discriminant mirrors $r_{i}^{\perp}$ meets $H_{\chi}^{4}$ in the same $H^{2}$, namely $H_{\chi}^{4} \cap R^{\perp}$.

The following lemma asserts that these are the only ways that $H_{\chi}^{4}$ can meet $\mathcal{H}$. In terms of cubic surfaces, the first possibility parametrizes surfaces with a real node, while the second parametrizes surfaces with a complex conjugate pair of nodes.

Lemma 6.1. - Suppose $\chi$ is an anti-involution of $\Lambda$ and $M$ is a discriminant mirror with $M \cap H_{\chi}^{4} \neq \varnothing$. Then either

(i) $M \cap H_{\chi}^{4}$ is a copy of $H^{3}$, namely $H_{\chi}^{4} \cap r^{\perp}$ for a rootr of $\Lambda^{\chi}$ of norm 1 or 3 , or

(ii) $M \cap H_{\chi}^{4}$ is a copy of $H^{2}$, namely $H_{\chi}^{4} \cap R^{\perp}$ for a $G_{2}$ root system $R$ in $\Lambda^{\chi}$.

Conversely, if $r$ is a root of norm 1 or 3 in $\Lambda^{\chi}$ (resp. $R$ is a $G_{2}$ root system in $\Lambda^{\chi}$ ), then $H_{\chi}^{4} \cap r^{\perp}$ (resp. $H_{\chi}^{4} \cap R^{\perp}$ ) is the intersection of $H_{\chi}^{4}$ with some discriminant mirror.

Proof. - As a discriminant mirror, $M=r^{\perp}$ for some norm 1 vector $r$ of $\Lambda$. Since $M \cap H_{\chi}^{4} \neq \varnothing, M$ contains points fixed by $\chi$, so that $\chi(M)$ meets $M$, which is to say that $r^{\perp}$ meets $\chi(r)^{\perp}$. By Lemma 2.3, either $r^{\perp}=\chi(r)^{\perp}$ or $r \perp \chi(r)$. In the first case, $\chi$ preserves the $\mathcal{E}$-span of $r$. The anti-involutions of a rank one free $\mathcal{E}$-module are easy to understand: every one leaves invariant either a generator or $\theta$ times a generator. Therefore $\Lambda^{\chi}$ contains a unit multiple of $r$ or $\theta r$. Then conclusion (i) applies. In the second case, $r_{1}:=r$ and $r_{2}:=\chi(r)$ span a copy of $\mathcal{E}^{2}$ and $\Lambda^{\chi}$ contains the norm $2 \operatorname{roots} \alpha r_{1}+\bar{\alpha} r_{2}$ and norm $6 \operatorname{roots} \alpha \theta r_{1}+\bar{\alpha} \bar{\theta} r_{2}$, where $\alpha$ varies over the units of $\mathcal{E}$. These form a $G_{2}$ root system $R$ in $\Lambda^{\chi}$, and it is easy to see that

$$
M \cap H_{\chi}^{4}=M \cap \chi(M) \cap H_{\chi}^{4}=R^{\perp} \cap H_{\chi}^{4}
$$

is a copy of $H^{2}$. Therefore conclusion (ii) applies. 
The converse is easy: if $r$ is a root of $\Lambda^{\chi}$ of norm 1 or 3 then we take the discriminant mirror to be $r^{\perp}$, and if $R$ is a $G_{2}$ root system in $\Lambda^{\chi}$ then we take $M$ to be either $r_{1}^{\perp}$ or $r_{2}^{\perp}$ for $r_{1}$ and $r_{2}$ as in (6.1).

COROLlary 6.2. - For $j=0, \ldots, 4, H_{j}^{4} \cap \mathcal{H}$ is the union of the orthogonal complements of the discriminant roots of $\Lambda_{j}$ and the $G_{2}$ root systems in $\Lambda_{j}$.

For our applications we need to re-state this result in terms of the Weyl chamber $C_{j}$ for $W_{j}$ :

Lemma 6.3. - If $x \in C_{j}$ then $x \in \mathcal{H}$ if and only if either

(i) $x$ lies in $r^{\perp}$ for $r$ a simple root of $W_{j}$ of norm 1 or 3 , or

(ii) $x$ lies in $r^{\perp} \cap s^{\perp}$, where $r$ and $s$ are simple roots of $W_{j}$ of norms 2 and 6 , whose mirrors meet at angle $\pi / 6$.

Proof. - If 6.3 holds then $x$ obviously lies in $\mathscr{H}$. If 6.3 holds then the reflections in $r$ and $s$ generate a dihedral group of order 12, and the images of $r$ and $s$ under this group form a $G_{2}$ root system $R$ in $\Lambda_{j}$. Then $x \in \mathcal{H}$ by Lemma 6.1.

To prove the converse, suppose $x \in C_{j} \cap \mathcal{H}$. By Lemma 6.1, either $x \in r^{\perp}$ for a root $r$ of $\Lambda_{j}$ of norm 1 or 3 , or else $x \in R^{\perp}$ for a $G_{2}$ root system $R$ in $\Lambda_{j}$. We treat only the second case because the first is similar but simpler. We choose a set $\{r, s\}$ of simple roots for $R$, which necessarily have norms 2 and 6 and whose mirrors necessarily meet at angle $\pi / 6$. Then $r^{\perp}$ and $s^{\perp}$ are two of the walls for some Weyl chamber $C^{\prime}$ of $W_{j}$. This uses the fact that no two distinct mirrors of $W_{j}$ can meet, yet make an angle less than $\pi / 6$. (If there were such a pair of mirrors then there would be such a pair among the simple roots of $W_{j}$.) We apply the element of $W_{j}$ carrying $C^{\prime}$ to $C_{j}$; since $C_{j}$ is a fundamental domain for $W_{j}$ in the strong sense, this transformation fixes $x$. Then the images of $r$ and $s$ are simple roots of $W_{j}$ and the facets of $C_{j}$ they define both contain $x$.

We remark that all the triple bonds in Fig. 5.1 come from $G_{2}$ root systems, so the condition on the norms of $r$ and $s$ in part 6.3 of the lemma may be dropped. This leads to our final description of the moduli space of smooth real cubic surfaces:

THEOREM 6.4. - The moduli space $\mathcal{M}_{0}^{\mathbb{R}}$ falls into five components $\mathcal{M}_{0, j}^{\mathbb{R}}, j=0, \ldots, 4$. As a real analytic orbifold, $\mathcal{M}_{0, j}^{\mathbb{R}}$ is isomorphic to an open sub-orbifold of $P \Gamma_{j}^{\mathbb{R}} \backslash H_{j}^{4}$, namely the open subset obtained by deleting the images in $P \Gamma_{j}^{\mathbb{R}} \backslash H_{4}^{j}$ of the faces of $C_{j}$ corresponding to the blackened nodes $\bullet$ and triple bonds $\bigcirc=0$ of Fig. 1.1.

The two kinds of walls of the $C_{j}$ play such different roles that we will use the following language. In light of the theorem, a wall corresponding to a blackened node in Fig. 1.1 will be called a discriminant wall. The other walls will be called Eckardt walls, because the corresponding real cubic surfaces are exactly those that have real Eckardt points. (Eckardt points are not important in this paper; they just provide a convenient name for these walls. They are points through which pass three lines of the surface. The reader interested in more background, in particular the relation between Eckardt points and existence of automorphisms of order two, may consult [34, $\$ \$ 98,100$ and 101].) 


\section{Topology of the moduli space of smooth surfaces}

This section and the next two are applications of the theory developed so far. The theoretical development continues in Section 10.

The description of $\mathcal{M}_{0}^{\mathbb{R}}$ in Theorem 6.4 is so explicit that many facts about real cubic surfaces and their moduli can be read off the diagrams. In this section we give presentations of the orbifold fundamental groups $\pi_{1}^{\text {orb }}\left(\mathcal{M}_{0, j}^{\mathbb{R}}\right)$ of the components of $\mathcal{M}_{0}^{\mathbb{R}}$ and prove that the $\mathcal{M}_{0, j}^{\mathbb{R}}$ have contractible (orbifold) universal covers.

THEOREM 7.1. - The orbifold fundamental groups of the components of $\mathcal{M}_{0}^{\mathbb{R}}$ are:

$$
\begin{aligned}
& \pi_{1}^{\text {orb }}\left(\mathcal{M}_{0,0}^{\mathbb{R}}\right) \cong S_{5} \\
& \pi_{1}^{\text {orb }}\left(\mathcal{M}_{0,1}^{\mathbb{R}}\right) \cong\left(S_{3} \times S_{3}\right) \rtimes \mathbb{Z} / 2 \\
& \pi_{1}^{\text {orb }}\left(\mathcal{M}_{0,2}^{\mathbb{R}}\right) \cong\left(D_{\infty} \times D_{\infty}\right) \rtimes \mathbb{Z} / 2 \\
& \pi_{1}^{\text {orb }}\left(\mathcal{M}_{0,3}^{\mathbb{R}}\right) \cong \pi_{1}^{\text {orb }}\left(\mathcal{M}_{0,4}^{\mathbb{R}}\right) \cong \bigcirc
\end{aligned}
$$

where the $\mathbb{Z} / 2$ in each semidirect product exchanges the displayed factors of the normal subgroup.

Here $S_{n}$ is the symmetric group, $D_{\infty}$ is the infinite dihedral group, and the last group is a Coxeter group with the given diagram. We have labeled the leftmost bond " $\infty$ ", indicating the absence of a relation between two generators, rather than a strong or weak bond, because we are describing the fundamental group as an abstract group, not as a concrete reflection group. We remark that $\pi_{1}^{\text {orb }}\left(\mathcal{M}_{0,2}^{\mathbb{R}}\right)$ is isomorphic to the Coxeter group of the Euclidean $(2,4,4)$ triangle.

Proof of Theorem 7.1. - The general theory of Coxeter groups (see for example [17]) allows us to write down a presentation for $W_{j}$. The standard generators for $W_{j}$ are the reflections across the facets of $C_{j}$. Two of these reflections $\rho$ and $\rho^{\prime}$ satisfy $\left(\rho \rho^{\prime}\right)^{n}=1$ for $n=2$ (resp. 3,4 , or 6 ) if the corresponding nodes are joined by no bond (resp. a single bond, double bond, or triple bond). These relations and the relations that the generators are involutions suffice to define $W_{j}$.

We get a presentation of $\pi_{1}^{\text {orb }}\left(W_{j} \backslash\left(H_{j}^{4}-\mathcal{H}\right)\right)$ from the presentation of $W_{j}$ by omitting some of the generators and relations. Since the generators of $W_{j}$ correspond to the walls of $C_{j}$, and removing $\mathcal{H}$ from $C_{j}$ removes the discriminant walls, we leave out those generators. Since removing these walls also removes all the codimension two faces which are their intersections with other walls, we also leave out all the relations involving the omitted generators. Finally, we leave out the relations coming from triple bonds, because removing $\mathcal{H}$ from $C_{j}$ removes the codimension two faces corresponding to these bonds. For $j=0,4$ or $5, P \Gamma_{j}^{\mathbb{R}}=W_{j}$ and we can read off $\pi_{1}^{\text {orb }}\left(\mathcal{M}_{0, j}^{\mathbb{R}}\right)$ from the diagram, with the results given in the statement of the theorem. For $j=1$ or 2 the same computation shows that $\pi_{1}^{\text {orb }}\left(W_{j} \backslash\left(H_{j}^{4}-\mathcal{H}\right)\right)$ is $S_{3} \times S_{3}$ or $D_{\infty} \times D_{\infty}$. To describe $\pi_{1}^{\text {orb }}\left(\mathcal{M}_{0, j}^{\mathbb{R}}\right)$ one must take the semidirect product by the diagram automorphism. This action can also be read from Fig. 1.1. 
In the proof of the following theorem, the subgroup of $W_{j}$ generated by the reflections across the Eckardt walls of $C_{j}$ (the walls represented by hollow nodes in Fig. 1.1) plays a major role. We call it $W_{j}^{\text {Eck }}$. It has index 1 or 2 in a group $T_{j}$ that plays a major role in the next section.

THEOREM 7.2. - The $\mathcal{M}_{0, j}^{\mathbb{R}}$ are aspherical orbifolds, in the sense that their orbifold universal covers are contractible manifolds.

Proof. - We write $D_{j}$ for the component of $H_{j}^{4}-\mathscr{H}$ containing $C_{j}-\mathcal{H}$, and think of $\mathcal{M}_{0, j}^{\mathbb{R}}$ as

$$
\text { (the stabilizer } \left.T_{j} \text { of } D_{j} \text { in } P \Gamma_{j}^{\mathbb{R}}\right) \backslash D_{j} \text {. }
$$

Since $D_{j}$ is an orbifold cover of $\mathcal{M}_{0, j}^{\mathbb{R}}$, it suffices to show that $D_{j}$ is aspherical. One way to understand $D_{j}$ is as the union of the translates of $C_{j}-\mathcal{H}$ under $W_{j}^{\text {Eck }}$. Alternately, $W_{j}^{\text {Eck }}$ is the stabilizer of $D_{j}$ in $W_{j}$. Now we look at the $D_{j}$ individually. $W_{0}^{\text {Eck }}$ is the finite group $S_{5}$, and the four Eckardt walls are the walls containing a vertex $P$ of $C_{0}$. (Vertices in $H^{n}$ of an $n$-dimensional Coxeter polyhedron correspond bijectively to $n$-node subdiagrams of the Coxeter diagram which generate finite Coxeter groups.) Therefore $D_{0}$ is the interior of a finite-volume polyhedron centered at $P$, so $D_{0}$ is not just aspherical but even contractible. The same argument works for $j=1$, with $S_{3} \times S_{3}$ in place of $S_{5}$.

The case $j=2$ is more complicated, even though $W_{2}^{\text {Eck }}$ is still finite (the Coxeter group $G_{2} \times G_{2}$ ) and the Eckardt walls are still the walls meeting at a vertex $P$ of $C_{2}$. The complication is that the fixed-point set of each $G_{2}$ factor lies in $\mathcal{H}$. The result is that $D_{2}$ is the interior of a finite-volume polyhedron centered at $P$, minus its intersection with two mutually orthogonal $H^{2}$ 's that meet transversely at $P$. Therefore $D_{2}$ is homeomorphic to a product of two punctured open disks, so it is aspherical.

Now we will treat $j=3$; the case $j=4$ is just the same. What is new is that $W_{3}^{\text {Eck }}$ is infinite. However, one of the discriminant walls (the lower of the rightmost two in Fig. 1.1) is orthogonal to all of the Eckardt walls. Therefore $W_{3}^{\text {Eck }}$ preserves the hyperplane $H$ containing this discriminant wall. Furthermore, $W_{3}^{\text {Eck }}$ is the Coxeter group

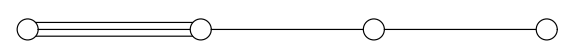

which is a nonuniform lattice in $\mathrm{PO}(3,1)$, acting on $H$ in the natural way. In particular, $H$ is a component of the boundary of $D_{3}$, and every $W_{3}^{\text {Eck }}$-translate of $C_{3}$ has one of its facets lying in $H$. Finally, $H$ is orthogonal to the codimension two face of $C_{3}$ associated to the triple bond in Fig. 1.1, and therefore orthogonal to all of its $W_{3}^{\text {Eck }}$-translates. We summarize: $D_{3}$ is the interior of an infinite-volume convex polyhedron in $H_{3}^{4}$, minus the union of a family of $H^{2}$ 's, each orthogonal to the distinguished facet $H$. Therefore $D_{3}$ is homeomorphic to the product of an open interval with $H-Z$, where $Z$ is the intersection of $H$ with the union of these $H^{2}$ 's.

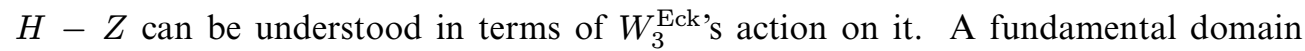
for $W_{3}^{\text {Eck }}$ is a simplex with shape described in (7.1), and the edge corresponding to the triple bond lies in $Z$. Indeed, $Z$ is the union of the $W_{3}^{\text {Eck }}$-translates of this edge. Direct visualization in hyperbolic 3-space shows that $Z$ is the union of countably many disjoint geodesics. Therefore $H^{3}-Z$ has the homotopy type of countably many circles, all identified at a point. This follows from stratified Morse theory; see Theorem 10.8 of [14]. 


\section{Relation with the work of B. Segre}

Classical knowledge about the topology of each connected component of the space of real smooth cubic forms was restricted to Segre's computation [34, $\$ 34-54]$ of the monodromy of the fundamental group of each component on the configuration of lines (real and complex) of a surface of that type. Our methods give a transparent calculation of this monodromy group $M_{j}$ over each component $\mathscr{C}_{0, j}^{\mathbb{R}}$, because the fundamental groups of these components are almost the same as the groups $\pi_{1}^{\text {orb }}\left(\mathcal{M}_{0, j}^{\mathbb{R}}\right)$ computed in the last section. In particular we show that four of Segre's computations are correct and correct an error in the remaining one.

Lemma 8.1. - For each $j=0, \ldots, 4$, there is an exact sequence

$$
1 \rightarrow \mathbb{Z} / 2 \rightarrow \pi_{1}\left(\mathscr{C}_{0, j}^{\mathbb{R}}\right) \rightarrow \pi_{1}^{\text {orb }}\left(\mathcal{M}_{0, j}^{\mathbb{R}}\right) \rightarrow \mathbb{Z} / 2 \rightarrow 1
$$

Here, the image of the middle map is the orientation-preserving subgroup of $\pi_{1}^{\text {orb }}\left(\mathcal{M}_{0, j}^{\mathbb{R}}\right)$ and the kernel is $\pi_{1}\left(G^{\mathbb{R}}\right)$.

For use in the proof and elsewhere in this section, we write $D_{j}$ for a component of $H_{j}^{4}-\mathcal{H}$ and $T_{j}$ for its stabilizer in $P \Gamma_{j}^{\mathbb{R}}$. This group is generated by the subgroup $W_{j}^{\text {Eck }}$ of $W_{j}$ introduced in Section 7, together with the diagram automorphism if one is present.

Proof sketch. - There are two ingredients. One is the exact homotopy sequence of the fibration $G^{\mathbb{R}} \rightarrow Y_{j} \rightarrow D_{j}$, where $Y_{j} \subseteq \mathcal{F}_{0}^{\mathbb{R}}$ is the $g^{\mathbb{R}}$-preimage of $D_{j}$. The other ingredient is the interaction of this sequence with the $T_{j}$-action on $Y_{j}$ and $D_{j}$. We omit the details. We remark that it would be more classical to consider $\pi_{1}\left(P \mathscr{C}_{0, j}^{\mathbb{R}}\right)$ instead. This would change the $\mathbb{Z} / 2=\pi_{1}\left(G^{\mathbb{R}}\right)$ on the left into $(\mathbb{Z} / 2)^{2}=\pi_{1}\left(P G^{\mathbb{R}}\right)$, but not affect our other considerations.

The monodromy of $\pi_{1}\left(\mathscr{C}_{0}, F\right)$ on lines is the classical map to the Weyl group $W\left(E_{6}\right) \cong \operatorname{Aut}(L(S), \eta(S))$, as in $\S 2.6$. As explained there, this is the same as the reduction modulo $\theta$ of the monodromy representation $\pi_{1}\left(\mathscr{C}_{0}\right) \rightarrow P \Gamma$. Therefore the monodromy of $\pi_{1}\left(\mathscr{C}_{0, j}^{\mathbb{R}}\right)$ on lines can be computed by taking the image of $\pi_{1}\left(\mathscr{C}_{0, j}^{\mathbb{R}}\right)$ in $P O(V)$.

By Lemma $8.1, \pi_{1}\left(\mathscr{C}_{0, j}^{\mathbb{R}}\right)$ acts by the orientation-preserving subgroup of $T_{j}$, so it will suffice to compute the map $T_{j} \rightarrow P O(V)$ and then pass to the image of the subgroup. Computing this map is very easy: one lifts each generator of $T_{j}$ to an element of $\Gamma$, reduces modulo $\theta$ to get an element of $O(V)$, and then passes to $P O(V)$. The ambiguity in the lift is unimportant because of the passage to $P O(V)$. One can work out the details in each case (see below for $j=2$ ), with the following result:

THEOREM 8.2. - Let $M_{j}$ denote the image of the monodromy representation $\pi_{1}\left(\mathscr{C}_{0, j}\right) \rightarrow W\left(E_{6}\right)$. Then

$$
\begin{aligned}
& M_{0} \cong A_{5} \\
& M_{1} \cong S_{3} \times S_{3} \\
& M_{2} \cong(\mathbb{Z} / 2)^{3} \rtimes \mathbb{Z} / 2 \\
& M_{3} \cong M_{4} \cong S_{4} .
\end{aligned}
$$

In $M_{2}, \mathbb{Z} / 2$ has fixed-point set $(\mathbb{Z} / 2)^{2}$ in $(\mathbb{Z} / 2)^{3}$, and this characterizes the group structure.

$4^{\mathrm{e}}$ SÉRIE - TOME $43-2010-\mathrm{N}^{\mathrm{o}} 1$ 
CAution. - It turns out that $\pi_{1}^{\text {orb }}\left(\mathcal{M}_{0,1}^{\mathbb{R}}\right) \cong\left(S_{3} \times S_{3}\right) \rtimes \mathbb{Z} / 2 \cong(\mathbb{Z} / 3)^{2} \rtimes D_{8}$ has two subgroups isomorphic to $S_{3} \times S_{3}$. The one which is the image of $\pi_{1}\left(\mathscr{C}_{0,1}^{\mathbb{R}}\right)$, here manifesting as $M_{1}$, is not the obvious one but the other one.

REMARK. - In the two cases where $\pi_{1}\left(\mathscr{C}_{0, j}^{\mathbb{R}}\right)$ is finite, namely $j=0$ or 1 , its representation in $W\left(E_{6}\right)$ is almost faithful. The kernel is precisely the central $\mathbb{Z} / 2=\pi_{1}\left(G^{\mathbb{R}}\right)$.

Our results confirm Segre's computation of $M_{0}, \ldots, M_{4}$, except for $M_{2}$, which he gives as $(\mathbb{Z} / 2)^{2}$ at the end of $\S 46$ (page 72 ). Our $M_{0}, \ldots, M_{4}$ are his $\Gamma_{1}, \ldots, \Gamma_{5}$, introduced in $\S 34$ and computed in $\$ 35$ to $\S 54$. In each case, he also gave a very detailed description of the action on various configurations of lines and tritangent planes of a surface in the appropriate component. We will show how to obtain this more detailed information from our perspective, in the case $j=2$.

By definition, $T_{2}=W_{2}^{\text {Eck }} \rtimes \mathbb{Z} / 2$ is generated by the reflections in $r_{1}, r_{3}, r_{5}$ and $r_{7}$ from the middle diagram in Fig. 5.1, together with the diagram automorphism. By the choice of roots, we already have lifts of the four reflections to $\Gamma$. For $r_{1}$ and $r_{5}$, reduction modulo $\theta$ gives the reflections of $V$ in the images of these two roots. The same applies to $r_{3}$ and $r_{7}$, except that one must divide them by $\theta$ before reducing modulo $\theta$. The point is that reflection in $r_{3}$ is the same as reflection in $r_{3} / \theta$, a primitive element of $\Lambda$. Therefore it acts on $V$ as the reflection in the image of $r_{3} / \theta$. We lift the diagram automorphism in the obvious way, to the isometry of $\Lambda$ that exchanges $r_{1} \leftrightarrow r_{5}, r_{3} \leftrightarrow r_{7}$ and fixes $r_{4}$.

So $M_{2}$ is the subgroup of $P O(V)$ generated by the diagram automorphism and the products of any evenly many reflections in the vectors $(0,1,-1,0,0),(0,0,0,1,-1),(1,0,0,0,0)$ and $(0,0,0,1,1)$, which are the reductions modulo $\theta$ of $r_{1}, r_{3} / \theta, r_{5}$ and $r_{7} / \theta$, respectively. These are mutually orthogonal, so $M_{2} \cong(\mathbb{Z} / 2)^{3} \rtimes(\mathbb{Z} / 2)$. The action of the diagram automorphism on $(\mathbb{Z} / 2)^{3}$ is easy to work out, with the result stated in Theorem 8.2.

Now we work out the action on lines. A key ingredient is the dictionary on p. 26 of [9] between the lines and tritangent planes of a cubic surface and certain objects in $V$. Namely, the tritangent planes of $S$ correspond to the "plus-points" of $P V$; with our choice of $q$, these are the lines $\langle v\rangle$ in $V$ with $q(v)=-1$ (see p. xii of [9]). And a line of $S$ corresponds to a "base", which with our choice of $q$ means a collection of five mutually orthogonal lines in $V$, each spanned by a vector $v$ with $q(v)=-1$. The fact that a base contains five plus-points corresponds to the fact that each line on $S$ is contained in 5 tritangent planes of $S$. One can check that each plus-point is contained in exactly 3 bases, corresponding to the fact that each tritangent plane contains 3 lines.

The anti-involution $\chi_{2}$ that defines the component $\mathscr{C}_{0,2}^{\mathbb{R}}$ acts on $V$ by

$$
\chi_{2}\left(x_{0}, \ldots, x_{4}\right)=\left(x_{0}, x_{1}, x_{2},-x_{3},-x_{4}\right) .
$$

This lets one work out which lines and tritangent planes are real. Together with our explicit generators for $M_{2}$, one can obtain extremely detailed results, for example:

THeOREM 8.3. - A real cubic surface of type $j=2$ has exactly five real tritangent planes. These have exactly one line $\ell$ in common, necessarily preserved by the monodromy group $M_{2}$. This group preserves exactly one of these five planes, and also each of the lines in it, which are real. Of the remaining four planes, two (say $\left.t_{1}, t_{2}\right)$ contain non-real lines and two (say $\left.t_{3}, t_{4}\right)$ contain only real lines. $M_{2}$ acts on these planes by $\mathbb{Z} / 2$, and contains an involution 
acting by $t_{1} \leftrightarrow t_{2}, t_{3} \leftrightarrow t_{4}$. The subgroup of $M_{2}$ that preserves each of $t_{1}, \ldots, t_{4}$ acts on the lines they contain as follows. It consists of every permutation of the form: for any evenly many of $t_{1}, \ldots, t_{4}$, in each of them exchange the two lines of $S$ other than $\ell$. The action of $M_{2} \cong(\mathbb{Z} / 2)^{3} \rtimes(\mathbb{Z} / 2)$ on these eight lines is faithful.

REMARK. - A careful reading of $\$ 46$ of [34] shows that Segre discusses actions of subgroups of $M_{2}$ on the set of four lines in $t_{1}$ and $t_{2}$, and on the set of four lines in $t_{3}$ and $t_{4}$, but does not seem to discuss the whole group. It is not clear how he reaches his conclusion that $M_{2} \cong(\mathbb{Z} / 2)^{2}$.

\section{Volumes}

In this section we compute the volume of each $P \Gamma_{j}^{\mathbb{R}} \backslash H_{j}^{4}$ by computing its orbifold Euler characteristic and using the general relation

$$
\operatorname{vol}(M)=\frac{\operatorname{vol}\left(S^{n}\right)}{\chi\left(S^{n}\right)}|\chi(M)|=\frac{2^{n} \pi^{n / 2}(n / 2) !}{n !}|\chi(M)|
$$

for a hyperbolic orbifold $M$ with $n=\operatorname{dim} M$ even. For the Euler characteristic, consider the subgroup $W_{j}$ generated by reflections, and its fundamental polyhedron $C_{j}$, described by its Coxeter diagram in Fig. 1.1. $W_{j}$ has index $\delta$ in $P \Gamma_{j}^{\mathbb{R}}$ with $\delta=1$ or 2 . The latter case occurs when the diagram has an automorphism of order two. Consider therefore the orbifold $W_{j} \backslash H_{j}^{4}$. Since

$$
\delta \cdot \chi\left(P \Gamma_{j}^{\mathbb{R}} \backslash H_{j}^{4}\right)=\chi\left(W_{j} \backslash H_{j}^{4}\right),
$$

it suffices to compute the right-hand side. To this end, consider a face $F$ of $C_{j}$ and its stabilizer $W_{j}(F)$ in $W_{j}$. If $\Phi$ stands for the set of proper faces of $C_{j}$, then

$$
\chi\left(W_{j} \backslash H_{j}^{4}\right)=1+\sum_{F \in \Phi} \frac{(-1)^{\operatorname{dim} F} \chi(F)}{\left|W_{j}(F)\right|}=1+\sum_{F \in \Phi} \frac{(-1)^{\operatorname{dim} F}}{\left|W_{j}(F)\right|} .
$$

Let $\Delta$ be a Coxeter diagram, let $\Sigma(\Delta)$ be the set of nonempty subdiagrams describing finite Coxeter groups, and for $E$ in $\Sigma$, let $|E|$ be the number of its nodes and $W(E)$ be the associated Coxeter group. The face of $C_{j}$ corresponding to $E$ has codimension $|E|$ in an even-dimensional space, so the previous equation can be written as

$$
\chi\left(W_{j} \backslash H_{j}^{4}\right)=1+\sum_{E \in \Sigma} \frac{(-1)^{|E|}}{|W(E)|} .
$$

For the Coxeter diagrams that occur in this paper, the enumeration of subdiagrams is lengthy but easy. We did the computations by hand and then checked them with a computer. Consider, for instance, the case of $W_{0}$. Every proper subdiagram describes a finite Coxeter group except the one got by omitting the rightmost node; for example, the other four-node subdiagrams (which describe vertices of $C_{0}$ ) have types $B_{4}, A_{1}^{2} \times A_{2}, A_{1} \times B_{3}$ and $A_{4}$. The resulting contribution to the Euler characteristic is

$$
(-1)^{4}\left(\frac{1}{2^{4} \cdot 4 !}+\frac{1}{2^{2} \cdot 3 !}+\frac{1}{2^{4} \cdot 3 !}+\frac{1}{5 !}\right)=\frac{121}{1920} .
$$

$4^{\text {e }}$ SÉRIE - TOME $43-2010-\mathrm{N}^{\mathrm{o}} 1$ 
Carrying out the full enumeration and computing the orders of the corresponding Weyl groups, one finds that

$$
\chi\left(P \Gamma_{0}^{\mathbb{R}} \backslash H^{4}\right)=1-\frac{5}{2}+\frac{17}{8}-\frac{11}{16}+\frac{121}{1920}=\frac{1}{1920} .
$$

This gives the first entry in table 1.1. The other calculations are similar.

\section{Moduli of stable complex cubic surfaces}

All of our discussions have been restricted to smooth cubic surfaces. However, one can still discuss moduli of singular surfaces, when the singularities are mild. In this section we recall from [1] the material necessary for our treatment in the next section of the moduli space of stable real cubic surfaces. Here stable means stable in the sense of geometric invariant theory (GIT). For cubic surfaces it is classical that this is simply the condition that the singularities be no worse than nodes (ordinary double points, $x^{2}+y^{2}+z^{2}=0$ in local analytic coordinates). See [1, (3.1)]. It is also classical that a cubic surface can have at most 4 nodes; see [7] for a modern treatment.

We write $\mathscr{C}_{s}$ for the space of all complex cubic forms defining stable surfaces. It contains $\mathscr{C}_{0}$, and a standard result from GIT is that $G$ acts properly on $\mathscr{C}_{s}$. Therefore $\mathcal{M}_{s}:=G \backslash \mathscr{C}_{s}$ is a complex-analytic orbifold as well as a quasi-projective variety. The main result of [1] is an isomorphism $\mathcal{M}_{s} \cong P \Gamma \backslash \mathbb{C} H^{4}$, extending the isomorphism $\mathcal{M}_{0} \cong P \Gamma \backslash\left(\mathbb{C} H^{4}-\mathcal{H}\right)$ of Theorem 3.3. For $\mathcal{M}_{s}$, this is an isomorphism of algebraic varieties, but not of orbifolds (see below).

Here are the main ideas behind this isomorphism; Theorem 10.2 is the precise statement. As explained in [1, (3.10) and (3.3)], the covering space $\mathcal{F}_{0} \rightarrow \mathscr{C}_{0}$ extends to a ramified covering space $\mathcal{F}_{s} \rightarrow \mathscr{C}_{s}$. Here $\mathcal{F}_{s}$ is the Fox completion (or normalization) of $\mathcal{F}_{0} \rightarrow$ $\mathscr{C}_{0}$ over $\mathscr{C}_{s}$, and we call its elements framed stable cubic forms. The naturality of this construction implies that the $G$ - and $P \Gamma$-actions on $\mathcal{F}_{0}$ extend to $\mathcal{F}_{s}$. The key facts about $\mathcal{F}_{s}$ are the following:

Lemma 10.1 ([1, (3.14)]). - G acts freely on $\mathcal{F}_{s}$, so $G \backslash \mathcal{F}_{s}$ is a complex manifold.

TheOREm 10.2 ([1, (3.17-19)]). - The period map $g: \mathcal{F}_{0} \rightarrow \mathbb{C} H^{4}$ extends to $\mathcal{F}_{s}$, factors through $G \backslash \mathcal{F}_{s}$, and induces a PГ-equivariant diffeomorphism $g: G \backslash \mathcal{F}_{s} \cong \mathbb{C} H^{4}$. It sends the $k$-nodal cubic surfaces to the locus in $\mathbb{C} H^{4}$ where exactly $k$ of the hyperplanes of $\mathcal{H}$ meet. Furthermore, the induced map

$$
\mathcal{M}_{s}=G \backslash \mathscr{C}_{s}=(G \times P \Gamma) \backslash \mathcal{F}_{s} \rightarrow P \Gamma \backslash \mathbb{C} H^{4}
$$

is an isomorphism of analytic spaces, but not of complex orbifolds.

We will need the following local description of $\mathcal{F}_{s} \rightarrow \mathscr{C}_{s}$ in the next section, and give a refinement of it in Lemma 11.1. 
Lemma 10.3 ([1, (3.10)]). - Suppose $f \in \mathcal{F}_{s}$ lies over $F \in \mathscr{C}_{s}$, the cubic surface $S$ defined by $F$ has $k$ nodes, and $x:=g(f) \in \mathbb{C} H^{4}$.

Then there exist coordinates $t_{1}, \ldots, t_{4}$ on $\mathbb{C} H^{4}$ which identify it with the unit ball in $\mathbb{C}^{4}$ and $x$ with the origin, whose pullbacks to $\mathcal{F}_{s}$ can be extended to local coordinates $t_{1}, \ldots, t_{20}$ around $f$, such that

(i) The components of $\mathcal{H}$ passing through $x$ are defined by $t_{1}=0, \ldots, t_{k}=0$.

(ii) The stabilizer $P \Gamma_{f}$ of $f$ is $(\mathbb{Z} / 6)^{k}$, acting on $\mathcal{F}_{s}$ by multiplying $t_{1}, \ldots, t_{k}$ by sixth roots of unity and leaving $t_{k+1}, \ldots, t_{20}$ invariant.

(iii) The functions $u_{1}=t_{1}^{6}, \ldots, u_{k}=t_{k}^{6}, u_{k+1}=t_{k+1}, \ldots, u_{20}=t_{20}$ are local coordinates around $F \in \mathscr{C}_{s}$.

(iv) The discriminant $\Delta \subseteq \mathscr{C}_{s}$ near $F$ is the union of the hypersurfaces $u_{1}=0, \ldots, u_{k}=0$.

In these coordinates, the period map $g: \mathcal{F}_{s} \rightarrow \mathbb{C} H^{4}$ is given near $f$ by forgetting $t_{5}, \ldots, t_{20}$.

The failure of the variety isomorphism $\mathcal{M}_{s} \cong P \Gamma \backslash \mathbb{C} H^{4}$ to be an orbifold isomorphism arises because of the presence of the $(\mathbb{Z} / 6)^{k}$ ramification of $\mathcal{F}_{s} \rightarrow \mathscr{C}_{s}$, described in parts (ii) and (iii) of this lemma. This is explained in more detail in [1, (3.18)]. We showed in [1, (3.19-20)] how to modify the orbifold structure of $P \Gamma \backslash \mathbb{C} H^{4}$ so that its identification with $\mathcal{M}_{s}$ becomes an orbifold isomorphism. The ramification of $\mathcal{F}_{s} \rightarrow \mathscr{C}_{s}$ will be the main issue in our treatment of stable real surfaces. Although we will not strictly need the results of $[1,(3.18-20)]$, the ideas they embody will play a major role in our analysis.

\section{Moduli of stable real cubic surfaces}

The goal of this section is to understand the moduli space $\mathcal{M}_{s}^{\mathbb{R}}$ of stable real cubic surfaces as a quotient of real hyperbolic space $H^{4}$. In the previous section we defined $\mathscr{C}_{s}$ as the space of forms defining GIT-stable cubic surfaces, and recalled that $G$ acts properly on it. Therefore $G^{\mathbb{R}}$ acts properly on $\mathscr{C}_{s}^{\mathbb{R}}:=\mathscr{C}_{s} \cap \mathscr{C}^{\mathbb{R}}$. We denote the quotient by $\mathcal{M}_{s}^{\mathbb{R}}$, which is a real-analytic orbifold in a natural way. In the smooth case we were able to pass from the complex orbifold isomorphism $\mathcal{M}_{0} \cong P \Gamma \backslash\left(\mathbb{C} H^{4}-\mathcal{H}\right)$ to the real orbifold isomorphisms $\mathcal{M}_{0, j}^{\mathbb{R}} \cong P \Gamma_{j}^{\mathbb{R}} \backslash\left(H_{j}^{4}-\mathcal{H}\right)$ fairly easily. A very substantial complication in the stable case is that the isomorphism $\mathcal{M}_{s} \cong P \Gamma \backslash \mathbb{C} H^{4}$ is not an orbifold isomorphism (see the end of Section 10). Nevertheless we will find a real-hyperbolic orbifold structure on $\mathcal{M}_{s}^{\mathbb{R}}$ by identifying it with $P \Gamma^{\mathbb{R}} \backslash H^{4}$ for a suitable lattice $P \Gamma^{\mathbb{R}}$ in $\mathrm{PO}(4,1)$. It will be obvious that this structure agrees with the moduli-space orbifold structure on $\mathcal{M}_{0}^{\mathbb{R}}$.

It is possible to skip the theory of this section and construct $P \Gamma^{\mathbb{R}}$ by gluing together the 5 orbifolds $P \Gamma_{j}^{\mathbb{R}} \backslash H_{j}^{4}$ along their discriminant walls. There is an essentially unique way to do this that makes sense (the one in Section 12), and one obtains the orbifold $P \Gamma^{\mathbb{R}} \backslash H^{4}$. This is what we did at first, but this did not even give a proof that the resulting space is homeomorphic to $\mathcal{M}_{s}^{\mathbb{R}}$. The essential content of this section is to give an intrinsic definition of the hyperbolic structure on $\mathcal{M}_{s}^{\mathbb{R}}$. Then Section 12 plays the role of computing an orbifold structure already known to exist, rather than constructing it.

$4^{\mathrm{e}}$ SÉRIE - TOME $43-2010-\mathrm{N}^{\mathrm{o}} 1$ 


\subsection{The space of framed stable real surfaces; their moduli space $K$}

We define $\mathcal{F}_{s}^{\mathbb{R}}$ as the preimage of $\mathscr{C}_{s}^{\mathbb{R}}$ in $\mathcal{F}_{s}$, so that $\mathscr{C}_{s}^{\mathbb{R}}=P \Gamma \backslash \mathcal{F}_{s}^{\mathbb{R}}$. We will see that $\mathcal{F}_{s}^{\mathbb{R}}$ is not a manifold, because of the ramification of $\mathcal{F}_{s} \rightarrow \mathscr{C}_{s}$, but it is a union of embedded submanifolds. We define $K$ to be $G^{\mathbb{R}} \backslash \mathcal{F}_{s}^{\mathbb{R}}$, which is not a manifold either. At this point it is merely a topological space; below, we will equip it with a metric structure. Essentially by definition, $\mathcal{M}_{s}^{\mathbb{R}}$ coincides with $P \Gamma \backslash K$. If $K$ were a manifold then this would define an orbifold structure on $M_{s}^{\mathbb{R}}$. But it is not, so we must take a different approach. First we will give a local description of $\mathcal{F}_{s}^{\mathbb{R}} \subseteq \mathcal{F}_{s}$, and then show that $g: \mathcal{F}_{s} \rightarrow \mathbb{C} H^{4}$ induces a local embedding $K \rightarrow \mathbb{C} H^{4}$. This makes $K$ into a metric space, using the path metric obtained by pulling back the metric on $\mathbb{C} H^{4}$. Finally, we will study the action of $P \Gamma$ on $K$ to deduce that $P \Gamma \backslash K$, as a metric space, is locally modeled on quotients of $H^{4}$ by finite groups. Such a metric space has a unique hyperbolic orbifold structure. The completeness of this structure on $\mathcal{H}_{s}^{\mathbb{R}}$ then follows from the completeness of $P \Gamma \backslash \mathbb{C} H^{4}$, and orbifold uniformization then implies the existence of a discrete group $P \Gamma^{\mathbb{R}}$ acting on $H^{4}$ with $\mathcal{M}_{s}^{\mathbb{R}} \cong P \Gamma^{\mathbb{R}} \backslash H^{4}$. See Section 12 for a concrete description of $P \Gamma^{\mathbb{R}}$ and Section 13 for a proof that it is not arithmetic.

We begin with a local description of $\mathcal{F}_{s}^{\mathbb{R}}$. This requires a refinement of the local description of $\mathcal{F}_{s}$ given in Lemma 10.3.

Lemma 11.1. - Under the assumptions of Lemma 10.3, suppose F lies in $\mathscr{C}_{s}^{\mathbb{R}}$ and defines a surface with $2 a$ non-real and $b$ real nodes. Then the local coordinates of Lemma 10.3 on $\mathbb{C} H^{4}, \mathcal{F}_{s}$ and $\mathscr{C}_{s}$ may be chosen to also satisfy the following: near $F$, complex conjugation $\kappa: \mathscr{C}_{s} \rightarrow \mathscr{C}_{s}$ acts by

$$
u_{i} \mapsto \begin{cases}\bar{u}_{i+1} & \text { for } i \text { odd and } i \leq 2 a \\ \bar{u}_{i-1} & \text { for } i \text { even and } i \leq 2 a \\ \bar{u}_{i} & \text { for } i>2 a .\end{cases}
$$

Proof. - The coordinates $t_{1}, \ldots t_{k}$ of Lemma 10.3 are in one to one correspondence with the nodes of $S$ and, once a correspondence is fixed, each $t_{i}$ is unique up to multiplication by a complex number of absolute value one. The same is therefore true of the coordinates $u_{1}, \ldots u_{k}$. Since $\kappa$ permutes the sets $u_{i}=0$ in the same way that it permutes the nodes of $S$, namely interchanges complex conjugate nodes and preserves real ones, it is clear that the $t_{i}$ and hence the $u_{i}$ can be chosen so that $\kappa$ acts on $u_{1}, \ldots u_{k}$ as in (11.1). That the coordinates may be chosen so that $\kappa$ also acts this way on $u_{k+1}, \ldots, u_{20}$ can be derived from the fact that any anti-involution of a complex manifold in a neighborhood of any fixed point is modeled on complex conjugation of $\mathbb{C}^{n}$.

In these local coordinates, $\mathscr{C}_{s}^{\mathbb{R}}$ is the fixed-point set of $\kappa$. To describe $\mathcal{F}_{s}^{\mathbb{R}}$ near $f$, we simply compute the preimage of $\mathscr{C}_{s}^{\mathbb{R}}$. The most important cases are first, a single real node $(a=0$, $b=1)$, and second, a single pair of conjugate nodes $(a=1, b=0)$.

In the case of a single real node, $\mathcal{F}_{s}^{\mathbb{R}}$ near $f$ is modeled on a neighborhood of the origin in

$$
\left\{\left(t_{1}, \ldots, t_{20}\right) \in \mathbb{C}^{20}: t_{1}^{6}, t_{2}, \ldots, t_{20} \in \mathbb{R}\right\} .
$$

That is, a neighborhood of $f$ is modeled on six copies of $\mathbb{R}^{20}$, glued together along a common $\mathbb{R}^{19}$. 
In the case of two complex conjugate nodes, $\mathcal{F}_{s}^{\mathbb{R}}$ near $f$ is modeled on a neighborhood of the origin in

$$
\left\{\left(t_{1}, \ldots, t_{20}\right) \in \mathbb{C}^{20}: t_{2}^{6}=\bar{t}_{1}^{6} \text { and } t_{3}, \ldots t_{20} \in \mathbb{R}\right\} .
$$

That is, on the union of six copies of $\mathbb{R}^{20}$, glued together along a common $\mathbb{R}^{18}$. The $\mathbb{R}^{18}$ is given by $t_{1}=t_{2}=0$ and maps diffeomorphically to $\Delta \cap \mathscr{C}_{s}^{\mathbb{R}}$, and each component of the complement is a six-fold cover of the part of $\mathscr{C}_{s}^{\mathbb{R}}-\Delta$ near $F$.

\subsection{Many different real structures}

We have defined $\mathcal{F}_{s}^{\mathbb{R}}$ as the preimage of $\mathscr{C}_{s}^{\mathbb{R}}$ in $\mathcal{F}_{s}$, but it is helpful to think of it as the union of the real loci of many different real structures. Namely, if $f \in \mathcal{F}_{0}^{\mathbb{R}}$ lies over $F \in \mathscr{C}_{0}^{\mathbb{R}}$ then there is a unique lift of the complex conjugation $\kappa$ of $\mathscr{C}_{s}$ to an anti-involution $\chi$ of $\mathcal{F}_{0}$ that fixes $f$. We saw this construction in $\S 3.3$, and wrote $\mathcal{F}_{0}^{\chi}$ for $\chi$ 's fixed points. (See also the last part of the proof of Theorem 3.3.)

The naturality of the Fox completion implies that $\chi$ extends to $\mathcal{F}_{s}$. Then the set $\mathcal{F}_{s}^{\chi}$ of $\chi$ 's fixed points is the real locus of one real structure, namely $\chi$. It is clear that $\mathcal{F}_{s}^{\mathbb{R}}$ is the union of the $\mathcal{F}_{s}^{\chi}$ as $\chi$ varies over the anti-involutions of $\mathcal{F}_{s}$ lying over $\kappa$. We have already seen this set of anti-involutions: it is $P \mathscr{C}$, the set of projective classes of anti-involutions of $\Lambda$ defined in $\S 3.3$.

\subsection{The family of isomorphisms $G^{\mathbb{R}} \backslash \mathcal{F}_{s}^{\chi} \rightarrow H_{\chi}^{4}$}

Recall from Section 2.4 that $g: \mathcal{F}_{s} \rightarrow \mathbb{C} H^{4}$ is the complex period map. Lemma 11.3 below is the extension of the diffeomorphism $G^{\mathbb{R}} \backslash \mathcal{F}_{0}^{\chi} \cong H_{\chi}^{4}-\mathcal{H}$ of Theorem 3.3 to $G^{\mathbb{R}} \backslash \mathcal{F}_{s}^{\chi} \cong H_{\chi}^{4}$; to prove it we need the following general principle.

Lemma 11.2. - Let $G$ be a Lie group acting properly and with finite stabilizers on a smooth manifold $X$, let $F$ be a finite group of diffeomorphisms of $X$ normalizing $G$, let $X^{F}$ be its fixed-point set, and let $G^{F}$ be its centralizer in $G$. Then the natural map $G^{F} \backslash X^{F} \rightarrow G \backslash X$ is proper.

Proof. - We write $\pi$ and $\pi^{F}$ for the maps $X \rightarrow G \backslash X$ and $X^{F} \rightarrow G^{F} \backslash X^{F}$, and $f$ for the natural map $G^{F} \backslash X^{F} \rightarrow G \backslash X$. We prove the theorem under the additional hypothesis that $F$ and $G$ meet trivially; this is all we need and the proof in the general case is similar. This hypothesis implies that the group $H$ generated by $G$ and $F$ is $G \rtimes F$. Begin by choosing a complete $H$-invariant Riemannian metric on $X$.

To prove $f$ proper it suffices to exhibit for any $G$-orbit $\Theta \subseteq X$ a $G$-invariant neighborhood $U \subseteq X$ with $f^{-1}(\pi(U))$ precompact. Since $G$ has finite index in $H, \theta . H \subseteq X$ is the union of finitely many $G$-orbits. Using properness and Riemannian geometry one finds $\varepsilon>0$ such that (1) distinct $G$-orbits in $\theta . H$ lie at distance $>\varepsilon$, and (2) any point of $X$ at distance $<\varepsilon$ from $\theta$ has a unique nearest point in $\theta$. We take $U$ to be the open $\varepsilon / 2$-neighborhood of $\theta$.

To show that $f^{-1}(\pi(U))$ is precompact we will exhibit a compact set $K \subseteq X^{F}$ with $\pi^{F}(K)$ containing $f^{-1}(\pi(U))$. We claim that there are finitely many $G^{F}$-orbits in $\Theta \cap X^{F}$, so we can choose orbit representatives $\tilde{x}_{1}, \ldots, \tilde{x}_{n}$. If $\Theta \cap X^{F}$ is empty then this is trivial. If $\theta \cap X^{F}$ is 
nonempty, say containing $\tilde{x}$, then the $G^{F}$-orbits in $\Theta \cap X^{F}$ are in bijection with the conjugacy classes of splittings of

$$
1 \rightarrow G_{\tilde{x}} \rightarrow G_{\tilde{x}} \rtimes F \rightarrow F \rightarrow 1,
$$

where $G_{\tilde{x}}$ is the $G$-stabilizer of $\tilde{x}$. Since $G_{\tilde{x}}$ is finite, there are finitely many splittings, hence finitely many orbits. We take $K$ to be the union of the closed $\varepsilon / 2$-balls around $\tilde{x}_{1}, \ldots, \tilde{x}_{n}$, intersected with $X^{F}$. (In particular, $K$ is empty if $\theta \cap X^{F}$ is.)

$K$ is obviously compact, so all that remains is to prove $f^{-1}(\pi(U)) \subseteq \pi^{F}(K)$. If $f^{-1}(\pi(U))$ is empty then we are done. Otherwise, suppose $y \in f^{-1}(\pi(U)) \subseteq G^{F} \backslash X^{F}$ and let $\tilde{y} \in X^{F}$ lie over it. Now, $\tilde{y}$ is $F$-invariant and $F$ permutes the $G$-orbits in $\Theta$.H. Since $\tilde{y}$ lies within $\varepsilon / 2$ of $\Theta$, it lies at distance $>\varepsilon / 2$ of every other $G$-orbit in $\Theta$. $H$, so $F$ preserves $\vartheta$. Therefore $F$ preserves the unique point $\tilde{x}$ of $\theta$ closest to $\tilde{y}$, so $\tilde{x} \in X^{F}$. We choose $g \in G^{F}$ with $\tilde{x} . g$ equal to one of the $\tilde{x}_{i}$. Then $\tilde{y} . g$ lies within $\varepsilon / 2$ of $\tilde{x} . g=\tilde{x}_{i}$, hence lies in $K$, and $\pi^{F}(\tilde{y} . g)=y$, proving $f^{-1}(\pi(U)) \subseteq \pi^{F}(K)$.

Lemma 11.3. - For every $\chi \in P^{\mathscr{G}}$, the restriction of the period map $g: \mathcal{F}_{s} \rightarrow \mathbb{C} H^{4}$ to $\mathcal{F}_{s}^{\chi}$ defines an isomorphism $G^{\mathbb{R}} \backslash \mathcal{F}_{s}^{\chi} \cong H_{\chi}^{4}$ of real-analytic manifolds.

Proof. - It is a local diffeomorphism because its rank is everywhere 4 by Theorem 10.2. Injectivity follows from the argument used for Theorem 3.3; this uses the freeness of the $G$ action on $\mathcal{F}_{s}$, see Lemma 10.1 . To see surjectivity, we apply the previous lemma with $G=G, X=\mathcal{F}_{s}, F=\{1, \chi\}, X^{F}=\mathcal{F}_{s}^{\chi}$ and $G^{F}=G^{\mathbb{R}}$. Therefore the map $G^{\mathbb{R}} \backslash \mathcal{F}_{s}^{\chi} \rightarrow G \backslash \mathcal{F}_{s} \cong \mathbb{C} H^{4}$ is proper, so its image is closed. Theorem 3.3 tells us that the image contains the open dense subset $g\left(\mathcal{F}_{0}^{\chi}\right)=H_{\chi}^{4}-\mathcal{H}$, so the map is surjective.

\subsection{The local embedding $K \rightarrow \mathbb{C} H^{4}$}

The purpose of this subsection is to show that the complex period map $g$ defines a local embedding $K=G^{\mathbb{R}} \backslash \mathcal{F}_{s}^{\mathbb{R}} \rightarrow \mathbb{C} H^{4}$, which we will use in the next subsection to define a piecewise-hyperbolic metric on $K$.

Lemma 11.4. - Suppose $f \in \mathcal{F}_{s}^{\mathbb{R}}$, and $\alpha_{1}, \ldots, \alpha_{\ell}$ are the elements of $P \mathscr{C}$ that fix $f$. Then the map

$$
G^{\mathbb{R}} \backslash\left(\cup_{i=1}^{\ell} \mathcal{F}_{s}^{\alpha_{i}}\right) \rightarrow \cup_{i=1}^{\ell} H_{\alpha_{i}}^{4}
$$

induced by $g$ is a homeomorphism.

The left side of (11.4) contains a neighborhood of the image of $f$ in $K$, so the lemma implies that $g: K \rightarrow \mathbb{C} H^{4}$ is a local embedding. We will write $K_{f}$ for the right side of (11.4). It is the part of $K$ relevant to $f$.

Before giving the proof, we observe that it is easy to work out formulas for $\alpha_{1}, \ldots, \alpha_{\ell}$ in the local coordinates $t_{1}, \ldots, t_{20}$ on $\mathcal{F}_{s}$. Since Lemma 11.1 gives a formula for $\kappa$ near $F \in \mathscr{C}_{s}^{\mathbb{R}}$ in the local coordinates $u_{1}, \ldots, u_{20}$, and $\mathcal{F}_{s} \rightarrow \mathscr{C}_{s}$ is given by $u_{1}=t_{1}^{6}, \ldots, u_{k}=t_{k}^{6}$, $u_{k+1}=t_{k+1}, \ldots, u_{20}=t_{20}$, one can simply write down the lifts of $\kappa$. For example, in the one-real-node case there are 6 lifts, given by

$$
\left(t_{1}, \ldots, t_{20}\right) \mapsto\left(\bar{t}_{1} \zeta^{i}, \bar{t}_{2}, \ldots, \bar{t}_{20}\right),
$$


where $\zeta=e^{\pi i / 3}$, and in the conjugate-pair case there are also 6 lifts, given by

$$
\left(t_{1}, \ldots, t_{20}\right) \mapsto\left(\bar{t}_{2} \zeta^{i}, \bar{t}_{1} \zeta^{i}, \bar{t}_{3}, \ldots, \bar{t}_{20}\right) .
$$

Proof of Lemma 11.4. - We first claim that for all $i$ and $j$,

$$
g: \mathcal{F}_{s}^{\alpha_{i}} \cap \mathcal{F}_{s}^{\alpha_{j}} \rightarrow H_{\alpha_{i}}^{4} \cap H_{\alpha_{j}}^{4}
$$

is surjective. To see this, let $C$ be the component of $\mathcal{F}_{s}^{\alpha_{i}} \cap \mathcal{F}_{s}^{\alpha_{j}}$ containing $f$. This is a component of the fixed-point set of the finite group generated by $\alpha_{i}$ and $\alpha_{j}$. In particular, it is a smooth manifold whose tangent spaces are all totally real. Since $C$ is connected, its dimension everywhere is its dimension at $f$, which by our local coordinates is $16+$ $\operatorname{dim}_{\mathbb{R}}\left(H_{\alpha_{i}}^{4} \cap H_{\alpha_{j}}^{4}\right)$. Since the tangent spaces are totally real and the kernel of the derivative of the period map has complex dimension 16, the (real) rank of $\left.g\right|_{C}$ equals $\operatorname{dim}_{\mathbb{R}}\left(H_{\alpha_{i}}^{4} \cap H_{\alpha_{j}}^{4}\right)$ everywhere. Therefore $g(C)$ is open. It is also closed, since $g$ induces a diffeomorphism from each $G^{\mathbb{R}} \backslash \mathcal{F}_{s}^{\alpha_{i}}$ to $H_{\alpha_{i}}^{4}$ for each $i$. This proves surjectivity, since $H_{\alpha_{i}}^{4} \cap H_{\alpha_{j}}^{4}$ is connected.

Now we prove the lemma itself; the map (11.4) is surjective and proper because $G^{\mathbb{R}}$ $\mathcal{F}_{s}^{\alpha_{i}} \rightarrow H_{\alpha_{i}}^{4}$ is surjective and proper for each $i$. To prove injectivity, suppose $a_{i} \in G^{\mathbb{R}} \backslash \mathcal{F}_{s}^{\alpha_{i}}$ for $i=1,2$ have the same image in $\mathbb{C} H^{4}$. Then their common image lies in $H_{\alpha_{1}}^{4} \cap H_{\alpha_{2}}^{4}$, so by the claim above there exists $b \in G^{\mathbb{R}} \backslash\left(\mathcal{F}_{s}^{\alpha_{i}} \cap \mathcal{F}_{s}^{\alpha_{j}}\right)$ with the same image. Since each $G^{\mathbb{R}} \backslash \mathcal{F}_{s}^{\alpha_{i}} \rightarrow H_{\alpha_{i}}^{4}$ is injective, each $a_{i}$ coincides with $b$, so $a_{1}=a_{2}$.

\subsection{The local metric structure on $P \Gamma \backslash K \cong \mathcal{M}_{s}^{\mathbb{R}}$}

At this point we know that $g$ locally embeds $K=G^{\mathbb{R}} \backslash \mathcal{F}_{s}^{\mathbb{R}}$ into $\mathbb{C} H^{4}$, and even have an identification of small open sets in $K$ with open sets in unions of copies of $H^{4}$ in $\mathbb{C} H^{4}$. The induced path-metric on $K$ is the largest metric which preserves the lengths of paths; under it, $K$ is piecewise isometric to $H^{4}$. $K$ is not a manifold - it may be described locally by suppressing the coordinates $t_{5}, \ldots, t_{20}$ from our local description of $\mathcal{F}_{s}^{\mathbb{R}}$. (See (11.2) and (11.3) for two examples.) Nevertheless, Corollary 11.6 below shows us that the path metric on $\mathcal{M}_{s}^{\mathbb{R}}=P \Gamma \backslash K$ is locally isometric to quotients of $H^{4}$ by finite groups. This is the key to defining the hyperbolic orbifold structure on $\mathcal{M}_{s}^{\mathbb{R}}$.

Our goal is to prove Corollary 11.6, that every point of $P \Gamma \backslash K$ has a neighborhood modeled on $H^{4}$ modulo a finite group. This requires a careful analysis with several different subgroups of $P \Gamma$ associated to $f \in \mathcal{G}_{s}^{\mathbb{R}}$. One of them is $A_{f}$, the subgroup of $P \Gamma$ fixing the image of $f$ in $K=G^{\mathbb{R}} \backslash \mathcal{F}_{s}^{\mathbb{R}}$. This contains $P \Gamma_{f} \cong(\mathbb{Z} / 6)^{k}$, often strictly. The third group is $B_{f}$, the subgroup of $P \Gamma_{f}$ generated by the order 6 complex reflections associated to the real nodes of $S$, rather than all the nodes. (The complex reflections are the elements of $P \Gamma_{f}$ that act on only one of the $t_{j}$.)

Lemma 11.4 says that

$$
A_{f} \backslash K_{f}=\left(A_{f} \times G^{\mathbb{R}}\right) \backslash\left(\cup_{i=1}^{\ell} \mathcal{F}_{s}^{\alpha_{i}}\right) \rightarrow\left(P \Gamma \times G^{\mathbb{R}}\right) \backslash \mathcal{F}_{s}^{\mathbb{R}}=\mathcal{M}_{s}^{\mathbb{R}}
$$

is a homeomorphism in a neighborhood of the image of $f$ in $K_{f} \subseteq K$, where $\alpha_{1}, \ldots, \alpha_{\ell}$ are as in that lemma. Therefore it suffices to study $A_{f} \backslash K_{f}$. It turns out that this is best done by first treating the intermediate quotient $B_{f} \backslash K_{f}$. 
So our next goal is to understand $B_{f} \backslash K_{f}$ in coordinates. The all-nodes-real case is much simpler than the general case, and should allow the reader to understand all the ideas in the rest of this section.

Lemma 11.5. - If $S$ has only real nodes, then $B_{f} \backslash K_{f}$ is isometric to $H^{4}$. If $S$ has a single pair of conjugate nodes, and possibly also some real nodes, then $B_{f} \backslash K_{f}$ is isometric to the union of six copies of $H^{4}$ identified along a common $H^{2}$. If $S$ has two pairs of conjugate nodes then $B_{f} \backslash K_{f}=K_{f}$ is the union of 36 copies of $H^{4}$, any two of which meet along an $H^{2}$ or at a point.

In each case, $A_{f} / B_{f}$ acts transitively on the indicated $H^{4}$ 's in $B_{f} \backslash K_{f}$. If $H$ is any one of them, and $\left(A_{f} / B_{f}\right)_{H}$ its stabilizer, then the natural map

$$
\left(A_{f} / B_{f}\right)_{H} \backslash H \rightarrow\left(A_{f} / B_{f}\right) \backslash\left(B_{f} \backslash K_{f}\right)=A_{f} \backslash K_{f}
$$

is an isometry of path metrics.

COROLlary 11.6. - Every point of $P \Gamma \backslash K$ has a neighborhood isometric to the quotient of an open set in $H^{4}$ by a finite group of isometries.

Proof. - The left term of (11.6) is a quotient of $H^{4}$ by a finite group, and the right term contains a neighborhood of the image of $f$ in $P \Gamma \backslash K$. The corollary follows from the fact that (11.6) is a local isometry.

Proof of Lemma 11.5. - We take $x=g(f)$ as before and refer to the coordinates $t_{1}, \ldots, t_{4}$ from Lemma 10.3 that identify $\mathbb{C} H^{4}$ with $B^{4}$. Recall that the cubic surface $S$ has $2 a$ non-real and $b$ real nodes, with $k=2 a+b$. The stabilizer $P \Gamma_{f}$ of $f$ in $P \Gamma$ acts on $\mathbb{C} H^{4}$ by multiplying $t_{1}, \ldots, t_{k}$ by $6^{\text {th }}$ roots of unity, and $B_{f}$ acts by multiplying $t_{2 a+1}, \ldots, t_{2 a+b}$ by $6^{\text {th }}$ roots of unity. $K_{f}$ may be described in the manner used to obtain (11.2) and (11.3), with $t_{5}, \ldots, t_{20}$ omitted. With concrete descriptions of $K_{f}$ and $B_{f}$ in hand, one can work out $B_{f} \backslash K_{f}$. Here are the results for the various cases.

First suppose $S$ has only real nodes. Then

$$
K_{f}=\left\{\left(t_{1}, \ldots, t_{4}\right) \in B^{4}: t_{1}^{6}, \ldots, t_{k}^{6}, t_{k+1}, \ldots, t_{4} \in \mathbb{R}\right\} .
$$

Each of the $2^{k}$ subsets

$$
K_{f, \varepsilon_{1}, \ldots, \varepsilon_{k}}=\left\{\left(t_{1}, \ldots, t_{4}\right) \in B^{4}: i^{\varepsilon_{1}} t_{1}, \ldots, i^{\varepsilon_{k}} t_{k} \in[0, \infty) \text { and } t_{k+1}, \ldots, t_{4} \in \mathbb{R}\right\},
$$

indexed by $\varepsilon_{1}, \ldots, \varepsilon_{k} \in\{0,1\}$, is isometric to the closed region in $H^{4}$ bounded by $k$ mutually orthogonal hyperplanes. Their union $U$ is a fundamental domain for $B_{f}$ in the sense that it maps homeomorphically and piecewise-isometrically onto $B_{f} \backslash K_{f}$. Under its path metric, $U$ is isometric to $H^{4}$, say by the following map, defined separately on each $K_{f, \varepsilon_{1}, \ldots, \varepsilon_{k}}$ by

$$
\left(t_{1}, \ldots, t_{k}\right) \mapsto\left(-i^{\varepsilon_{1}} t_{1}, \ldots,-i^{\varepsilon_{k}} t_{k}, t_{k+1}, \ldots, t_{4}\right) .
$$

This identifies $B_{f} \backslash K_{f}$ with the standard $H^{4}$ in $\mathbb{C} H^{4}$.

If $S$ has a single pair of non-real nodes and no real nodes, then $B_{f}$ is trivial and $B_{f} \backslash K_{f}=K_{f}$. The $\alpha_{i}$ are the 6 maps

$$
\alpha_{i}:\left(t_{1}, \ldots, t_{4}\right) \mapsto\left(\bar{t}_{2} \zeta^{i}, \bar{t}_{1} \zeta^{i}, \bar{t}_{3}, \bar{t}_{4}\right)
$$


with $i \in \mathbb{Z} / 6$, whose fixed-point sets are

$$
H_{\alpha_{i}}^{4}=\left\{\left(t_{1}, \ldots, t_{4}\right) \in B^{4}: t_{2}=\bar{t}_{1} \zeta^{i} \text { and } t_{3}, t_{4} \in \mathbb{R}\right\} .
$$

It is obvious that any two of these $H^{4}$ 's meet along the $H^{2} \subseteq B^{4}$ described by $t_{1}=t_{2}=0$ and $t_{3}, t_{4} \in \mathbb{R}$.

If $S$ has two pairs of non-real nodes (hence no real nodes at all) then the argument is essentially the same. The difference is that there are now 36 anti-involutions

$$
\alpha_{m, n}:\left(t_{1}, \ldots, t_{4}\right) \mapsto\left(\bar{t}_{2} \zeta^{m}, \bar{t}_{1} \zeta^{m}, \bar{t}_{4} \zeta^{n}, \bar{t}_{3} \zeta^{n}\right),
$$

where $m, n \in \mathbb{Z} / 6$, with fixed-point sets

$$
H_{\alpha_{m, n}}^{4}=\left\{\left(t_{1}, \ldots, t_{4}\right) \in B^{4}: t_{2}=\bar{t}_{1} \zeta^{m}, t_{4}=\bar{t}_{3} \zeta^{n}\right\} .
$$

If $\left(m^{\prime}, n^{\prime}\right) \neq(m, n)$ then $H_{\alpha_{m, n}}^{4}$ meets $H_{\alpha_{m^{\prime}, n^{\prime}}}^{4}$ in an $H^{2}$ if $m=m^{\prime}$ or $n=n^{\prime}$, and otherwise only at the origin.

If $S$ has a pair of non-real nodes and also a single real node then the argument is a mix of the cases above. $B_{f} \cong \mathbb{Z} / 6$ acts by multiplying $t_{3}$ by powers of $\zeta$, and there are 36 antiinvolutions, namely

$$
\alpha_{m, n}:\left(t_{1}, \ldots, t_{4}\right) \mapsto\left(\bar{t}_{2} \zeta^{m}, \bar{t}_{1} \zeta^{m}, \bar{t}_{3} \zeta^{n}, \bar{t}_{4}\right)
$$

We have

$$
K_{f}=\left\{\left(t_{1}, \ldots, t_{4}\right) \in B^{4}: t_{2}^{6}=\bar{t}_{1}^{6}, t_{3}^{6} \in \mathbb{R}, t_{4} \in \mathbb{R}\right\} .
$$

The union $U$ of the subsets with $t_{3}$ or $i t_{3}$ in $[0, \infty)$ is a fundamental domain for $B_{f}$; applying the identity map to the first subset and $t_{3} \mapsto-i t_{3}$ to the second identifies $U$ with

$$
\left\{\left(t_{1}, \ldots, t_{4}\right) \in B^{4}: t_{2}^{6}=\bar{t}_{1}^{6}, t_{3}, t_{4} \in \mathbb{R}\right\} .
$$

That is, $B_{f} \backslash K_{f}$ is what $K_{f}$ was in the case of no real nodes, as claimed. If $S$ has two non-real and two real nodes then the argument is only notationally more complicated.

The remaining claims are trivial unless there are non-real nodes. In every case, the transitivity of $A_{f} / B_{f}$ on the $H^{4}$ 's in $B_{f} \backslash K_{f}$ is easy to see because $P \Gamma_{f} \subseteq A_{f}$ contains transformations multiplying $t_{1}, \ldots, t_{2 a}$ by powers of $\zeta$. If $H$ is one of the $H^{4}$ 's and $J=$ $\left(A_{f} / B_{f}\right)_{H}$ is its stabilizer, then it remains to prove that $J \backslash H \rightarrow A_{f} \backslash K_{f}$ is an isometry. Surjectivity follows from the transitivity of $A_{f}$ on the $H^{4}$ 's. It is obviously a piecewise isometry, so all we must prove is injectivity. That is, if two points of $H$ are equivalent under $A_{f} / B_{f}$, then they are equivalent under $J$. To prove this it suffices to show that for all $y \in$ $B_{f} \backslash K_{f}$, the stabilizer of $y$ in $A_{f} / B_{f}$ acts transitively on the $H^{4}$ 's in $B_{f} \backslash K_{f}$ containing $y$. This is easy, using the stabilizer of $y$ in $P \Gamma_{f} / B_{f} \cong(\mathbb{Z} / 6)^{2 a}$.

\subsection{The hyperbolic orbifold structure}

We have equipped $\mathcal{M}_{s}^{\mathbb{R}}$ with a path metric which is locally isometric to quotients of $H^{4}$ by finite groups. It is easy to see that if $X$ is such a metric space then there is a unique realhyperbolic orbifold structure on $X$ whose path metric is the given one. (The essential point is that if $U$ and $U^{\prime}$ are connected open subsets of $H^{4}$ and $\Gamma$ and $\Gamma^{\prime}$ are finite groups of isometries of $H^{4}$ preserving $U$ and $U^{\prime}$ respectively, with $\Gamma \backslash U$ isometric to $\Gamma^{\prime} \backslash U^{\prime}$, then there is an isometry of $H^{4}$ carrying $U$ to $U^{\prime}$ and $\Gamma$ to $\Gamma^{\prime}$.) Therefore $\mathcal{M}_{s}^{\mathbb{R}}$ is a real hyperbolic orbifold. 
For completeness, we give explicit orbifold charts. Take $f$ as before, and $H$ one of the $H^{4}$ 's comprising $B_{f} \backslash K_{f}$. Recall that $\left(A_{f} / B_{f}\right)_{H}$ is its stabilizer in $A_{f} / B_{f}$. The orbifold chart is the restriction of the composition

$$
\begin{aligned}
H & \rightarrow\left(A_{f} / B_{f}\right)_{H} \backslash H \\
& \cong\left(A_{f} / B_{f}\right) \backslash\left(B_{f} \backslash K_{f}\right) \\
& =A_{f} \backslash K_{f} \\
& \cong\left(A_{f} \times G^{\mathbb{R}}\right) \backslash\left(\cup_{i=1}^{\ell} \mathcal{F}_{s}^{\alpha_{i}}\right) \\
& \rightarrow\left(P \Gamma \times G^{\mathbb{R}}\right) \backslash \mathcal{F}_{s}^{\mathbb{R}}=\mathcal{M}_{s}^{\mathbb{R}}
\end{aligned}
$$

to a suitable open subset of $H$. The homeomorphism of the second line is part of Lemma 11.5, and that of the fourth is Lemma 11.4. The map in the last line is a homeomorphism in a neighborhood $U$ of the image of $f$ in $\left(A_{f} \times G^{\mathbb{R}}\right) \backslash\left(\cup_{i=1}^{\ell} \mathcal{F}_{s}^{\alpha_{i}}\right)$. We take the domain of the orbifold chart to be the subset of $H$ which is the preimage of $U$.

THEOREM 11.7. - With the orbifold structure obtained by its identification with $P \Gamma \backslash K$, $M_{s}^{\mathbb{R}}$ is a complete real hyperbolic orbifold of finite volume, and there is a properly discontinuous group $P \Gamma^{\mathbb{R}}$ of motions of $H^{4}$ such that $\mathcal{M}_{s}^{\mathbb{R}}$ and $P \Gamma^{\mathbb{R}} \backslash H^{4}$ are isomorphic hyperbolic orbifolds.

Proof. - To prove $\mathcal{M}_{s}^{\mathbb{R}}$ complete, consider $K=G^{\mathbb{R}} \backslash \mathcal{F}_{s}^{\mathbb{R}}$. We know that $g$ maps $K$ to $\mathbb{C} H^{4}$; this is proper because any compact set in $\mathbb{C} H^{4}$ meets only finitely many $H_{\chi}^{4}, \chi \in P \mathscr{G}$, and $g$ carries each $G^{\mathbb{R}} \backslash \mathcal{F}_{s}^{\chi}$ homeomorphically to $H_{\chi}^{4}$ (Lemma 11.3). Since $K \rightarrow \mathbb{C} H^{4}$ is proper and $P \Gamma \backslash \mathbb{C} H^{4}$ is complete, so is $P \Gamma \backslash K$.

The uniformization theorem for complete hyperbolic orbifolds implies the existence of $P \Gamma^{\mathbb{R}}$ with the stated properties. See Proposition 13.3.2 of [35] or Chapter IIIG of [6] for discussion and proofs of this theorem. The volume of $M_{s}^{\mathbb{R}}$ is the sum of the volumes of the $P \Gamma_{j}^{\mathbb{R}} \backslash H_{j}^{4}$. Since these have finite volume, so does $\mathcal{M}_{s}^{\mathbb{R}}$.

REMARK. - It turns out that the analytic orbifold structures on $\mathcal{M}_{s}^{\mathbb{R}}$ coming from its definition as $G^{\mathbb{R}} \backslash \mathscr{C}_{s}^{\mathbb{R}}$ and its identification with $P \Gamma^{\mathbb{R}} \backslash H^{4}$ are different. But they do coincide on $\mathcal{M}_{0}^{\mathbb{R}}$. Also, the underlying topological orbifold structures coincide on a larger set, namely the complement of the locus of real surfaces having a conjugate pair of nodes. Along this locus, even the topological orbifold structures differ.

\section{A fundamental domain for $P \Gamma^{\mathbb{R}}$}

In the previous section we equipped the moduli space $\mathcal{M}_{s}^{\mathbb{R}}$ of stable real cubic surfaces with a complete hyperbolic orbifold structure, so $\mathcal{M}_{s}^{\mathbb{R}} \cong P \Gamma^{\mathbb{R}} \backslash H^{4}$ for some discrete group $P \Gamma^{\mathbb{R}}$. In this section we construct a fundamental domain and the associated generators for $P \Gamma^{\mathbb{R}}$. Besides its intrinsic interest, this allows us to prove in Section 13 that $P \Gamma^{\mathbb{R}}$ is nonarithmetic. Throughout this section, when we refer to $\mathcal{M}_{s}^{\mathbb{R}}$ as an orbifold, we refer to the hyperbolic structure. 


\subsection{The tiling of $H^{4}$ by chambers}

We begin by explaining how the orbifold universal cover $H \cong H^{4}$ of $\mathcal{M}_{s}^{\mathbb{R}}$ is tiled by copies of the polyhedra $C_{j}$ of Section 5. Consider the set of points in the orbifold $\mathcal{M}_{0}^{\mathbb{R}} \subseteq \mathcal{M}_{s}^{\mathbb{R}}$ whose local group contains no reflections, and its preimage under the orbifold covering map $H \rightarrow \mathcal{M}_{s}^{\mathbb{R}}$. Because the restriction of the hyperbolic structure of $\mathcal{M}_{s}^{\mathbb{R}}$ to $\mathcal{M}_{0}^{\mathbb{R}}$ is the (incomplete) structure described in Section 3, each component of the preimage is a copy of the interior of one of the $C_{j}$. We call the closure of such a component a chamber of type $j$. It is clear that the union of the chambers is $H$ and that their interiors are disjoint, so that they tile $H$.

Recall from Section 7 that we call a wall of a chamber a discriminant wall if it lies over the discriminant, and an Eckardt wall otherwise. By Theorem 6.4, it is a discriminant wall if and only if it corresponds to a blackened node of $C_{j}$ in Fig. 1.1. Because the orbifold structure on $\mathcal{M}_{s}^{\mathbb{R}}$ restricts to that on $\mathcal{M}_{0}^{\mathbb{R}}$, a generic point of an Eckardt wall is fixed by some reflection of $P \Gamma^{\mathbb{R}}$. Therefore $P \Gamma^{\mathbb{R}}$ contains the reflections across the Eckardt walls of the chambers. The same argument shows that if a chamber has type 1 or 2 , so that it has a diagram automorphism, then some element of $P \Gamma^{\mathbb{R}}$ carries it to itself by this automorphism.

We have seen that across any Eckardt wall of a chamber lies another chamber of the same type, in fact the mirror image of the first. Now we describe how the chambers meet across the discriminant walls. This is most easily understood by considering the 5 specific chambers $C_{j} \subseteq H_{j}^{4}$ given in Section 5, regarding all the $H_{j}^{4}$ 's as lying in $\mathbb{C} H^{4}$. Using the labeling of Fig. 5.1, we refer to the $k$ th simple root of $C_{j}$ as $r_{j k}$ and to the corresponding wall of $C_{j}$ as $C_{j k}$. The following lemma leads to complete information about how chambers meet across discriminant walls.

Lemma 12.1. - As subsets of $\mathbb{C} H^{4}$, we have $C_{04}=C_{14}, C_{13}=C_{24}, C_{22}=C_{34}$ and $C_{31}=C_{44}$. There is an element of $P \Gamma$ carrying $C_{37}$ isometrically to $C_{46}$.

Proof. - The first assertion is just a calculation; it is even easy if organized along the lines of the following treatment of the first equality. It is obvious that $r_{04}^{\perp} \subseteq H_{0}^{4}$ and $r_{14}^{\perp} \subseteq H_{1}^{4}$ coincide, since $r_{14}=\theta \cdot r_{04}$. Simple roots describing $C_{04}$ may be obtained by projecting the simple roots of $C_{0}$ into $r_{04}^{\perp}$, which amounts to setting the last coordinate equal to zero. Simple roots describing $C_{14}$ may be obtained by listing the walls of $C_{1}$ meeting $C_{14}$, namely $C_{11}, C_{12}, C_{13}$ and $C_{16}$, and projecting the corresponding roots into $r_{14}^{\perp}$, which again amounts to setting the last coordinate to zero. The two 4-tuples of vectors so obtained coincide, so they define the same polyhedron in $H_{0}^{4} \cap H_{1}^{4} \cong H^{3}$.

Now we prove the second claim. Since only two discriminant walls remain unmatched, we expect $C_{37}$ to coincide with some $P \Gamma$-translate of $C_{46}$. One can argue that this must happen, but it is easier to just find a suitable element $\gamma$ of $P \Gamma$. It should take $\theta r_{37}$ to $r_{46}$; it should also carry $r_{35}, r_{32}, r_{33}$ and $r_{36}$ to $r_{45}, r_{41}, r_{42}$ and $r_{43}$ in the order stated. These conditions 
determine $\gamma$, which turns out to be

$$
\gamma=\left(\begin{array}{ccrrr}
10+6 \omega & 4+2 \omega & 1-4 \omega & 1-4 \omega & 1-4 \omega \\
2-2 \omega & 1 & -2-2 \omega & -2-2 \omega & -2-2 \omega \\
1-4 \omega & -2 \omega & -2-2 \omega & -3-2 \omega & -3-2 \omega \\
1-4 \omega & -2 \omega & -3-2 \omega & -2-2 \omega & -3-2 \omega \\
1-4 \omega & -2 \omega & -3-2 \omega & -3-2 \omega & -2-2 \omega
\end{array}\right),
$$

where we regard vectors as column vectors and $\gamma$ acts on the left. Since $\gamma$ has entries in $\mathcal{E}$ and satisfies

$$
\gamma^{T} \cdot \operatorname{diag}[-1,1,1,1,1] \cdot \bar{\gamma}=\operatorname{diag}[-1,1,1,1,1],
$$

it lies in $P \Gamma$. By construction, it carries $C_{37}$ to $C_{46}$.

The lemma completes our picture of how the chambers meet along walls, as follows. Suppose for example that $P_{0}$ is a chamber of type 0 , so it has one discriminant wall, which we will call $P_{04}$. The lemma implies that $P_{04}$ is also a wall of a chamber of type 1 (say $P_{1}$ ). Furthermore, the relative positions of $P_{0}$ and $P_{1}$ can be determined by analyzing any nontrivial isometries of $P_{0}, P_{1}$ and their common wall. Applying this argument to the other cases of the lemma implies that every discriminant wall of a chamber is also a discriminant wall of another chamber, of known type. We will do this systematically in the next subsection.

\subsection{The polyhedron $Q$}

Now we construct what will turn out to be a fundamental domain for a subgroup $\frac{1}{2} P \Gamma^{\mathbb{R}}$ of index 2 in $P \Gamma^{\mathbb{R}}$. We choose a chamber $P_{0}$ of type 0 and write $P_{0 k}$ for its walls corresponding to the $C_{0 k}$ under the unique isometry $P_{0} \cong C_{0}$. (The detailed naming of walls is not needed for a conceptual understanding.) Across its discriminant wall $P_{04}$ lies a chamber $P_{1}$ of type 1; write $P_{1 k}$ for its walls corresponding to $C_{1 k}$ under the unique isometry $P_{1} \cong C_{1}$ that identifies $P_{04} \subseteq P_{1}$ with $C_{14}$. In particular, $P_{04}=P_{14}$. $P_{1}$ shares its discriminant wall $P_{17}$ with the image $P_{0}^{\prime}$ of $P_{0}$ under the diagram automorphism of $P_{1}$; we label the walls of $P_{0}^{\prime}$ by $P_{0 k}^{\prime}$ just as we did for $P_{0}$. We write $P_{2}$ for the chamber of type 2 on the other side of $P_{13}$. There are two isometries $P_{2} \cong C_{2}$, both of which identify $P_{13} \subseteq P_{2}$ with $C_{24}$, so we must work a little harder to fix our labeling of the walls of $P_{2}$. We choose the identification of $P_{2}$ with $C_{2}$ that identifies $P_{13} \cap P_{11} \subseteq P_{2}$ with $C_{24} \cap C_{21}$, and label the walls $P_{2 k}$ of $P_{2}$ accordingly. Now, $P_{2}$ has three discriminant walls: it shares $P_{24}$ with $P_{1}$, and across $P_{22}$ and $P_{26}$ lie chambers of type 3 . We write $P_{3}$ for the one across $P_{22}$ and $P_{3}^{\prime}$ for the one across $P_{26}$; these chambers are exchanged by the diagram automorphism. Label the walls of $P_{3}$ by $P_{3 k}$ according to the unique isometry $P_{3} \cong C_{3}$, and similarly for $P_{3}^{\prime}$. Finally, across $P_{31}$ lies a chamber $P_{4}$ of type 4 , whose walls we name $P_{4 k}$ according to the isometry $P_{4} \cong C_{4}$. Similarly, $P_{3}^{\prime}$ shares $P_{31}^{\prime}$ with a chamber $P_{4}^{\prime}$ which the diagram automorphism exchanges with $P_{4}$. We label the walls of $P_{4}^{\prime}$ accordingly. Let $Q$ be the union of all eight chambers $P_{0}, P_{0}^{\prime}$, $P_{1}, P_{2}, P_{3}, P_{3}^{\prime}, P_{4}$ and $P_{4}^{\prime}$. The construction of $Q$ is summarized in Fig. 12.1.

We remark that the diagram automorphisms of $P_{1}$ and $P_{2}$ coincide, in the sense that they are the same isometry of $H$, which we will call $S$; this isometry preserves $Q$. Throughout this section, "the diagram automorphism" refers to $S$. 


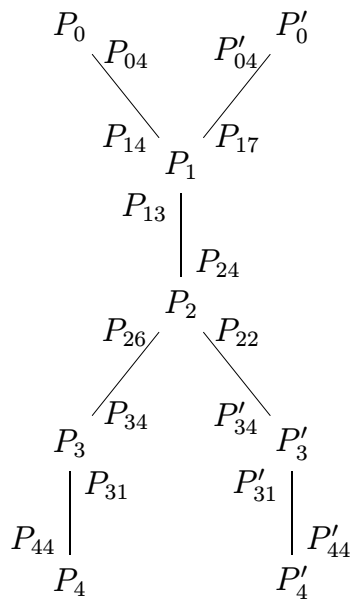

Figure 12.1. Assembly of the polyhedron $Q$ from 8 chambers.

\section{Lemma 12.2. - Q is a Coxeter polyhedron.}

Proof. - As a set, the boundary of $Q$ is the union of the Eckardt walls of the $P_{j}$ and $P_{j}^{\prime}$, together with $P_{37}, P_{46}, P_{37}^{\prime}$ and $P_{46}^{\prime}$. Suppose $W$ is an Eckardt wall of one of the $P_{j}$ or $P_{j}^{\prime}$ and $H_{W}^{3}$ is the hyperplane in $H$ that it spans. Then $Q$ lies entirely in one of the closed halfspaces bounded by $H_{W}^{3}$, because $P \Gamma^{\mathbb{R}}$ contains the reflection across $H_{W}^{3}$, while no point in the interior of $Q$ can be stabilized by a reflection of $P \Gamma^{\mathbb{R}}$. We call $H_{W}^{3} \cap Q$ an Eckardt wall of $Q$. Two Eckardt walls of $Q$ that meet make interior angle $\pi / n$ for some integer $n$, for otherwise some point in the interior of $Q$ would be stabilized by a reflection.

Now we claim that for $W=P_{37}, P_{46}, P_{37}^{\prime}$ or $P_{46}^{\prime}$, the wall of $Q$ containing $W$ coincides with $W$, and its only meetings with other walls of $Q$ are orthogonal intersections with Eckardt walls. We verify this for $W=P_{37}$; the key point is that $P_{37}$ is orthogonal to all the walls of $P_{3}$ that it meets, namely $P_{35}, P_{32}, P_{33}$ and $P_{36}$, and all these walls are Eckardt walls of $P_{3}$. By the above, we know that $Q$ lies in the region bounded by the $H^{3}$ 's containing $P_{35}, P_{32}, P_{33}$ and $P_{36}$, so the only walls of $Q$ which could meet $W$ are these walls (or rather their extensions to walls of $Q$ ). More precisely, there is a neighborhood of $P_{37}$ in $H$ whose intersection with $Q$ coincides with its intersection with $P_{3}$. All our claims follow from this. The same argument applies to $P_{46}$, and for the remaining two walls we appeal to symmetry.

\subsection{Simple roots for $Q$}

Since $Q$ is a Coxeter polyhedron, it may be described as the image in $H^{4}$ of the set of vectors $x$ having $x \cdot s \leq 0$ where $s$ varies over a set of simple roots for $Q$. There is one simple root for each wall of $Q$, so we may find simple roots for $Q$ by taking all the simple roots for the $P_{j}$ and $P_{j}^{\prime}$, and discarding the ones associated to the walls along which the $P_{j}$ and $P_{j}^{\prime}$ meet. We will also discard duplicates, which occur when walls of two different $P_{j}$ or $P_{j}^{\prime}$ lie in the same wall of $Q$.

$4^{\mathrm{e}}$ SÉRIE - TOME $43-2010-\mathrm{N}^{\mathrm{o}} 1$ 
Therefore we will need to know simple roots for all the $P_{j}$ and $P_{j}^{\prime}$. We identify $H$ with $H_{1}^{4} \subseteq \mathbb{C} H^{4}$, such that $P_{0}$ is $C_{0}$. Then $P_{1}$ is the image of $C_{1} \subseteq H_{1}^{4} \subseteq \mathbb{C} H^{4}$ under the map

$$
T_{1}:\left(x_{0}, \ldots, x_{4}\right) \mapsto\left(x_{0}, x_{1}, x_{2}, x_{3}, i x_{4}\right),
$$

which is an isometry of $\mathbb{C} H^{4}$ (although not an element of $P \Gamma$ ). This uses the facts that $C_{04}$ and $C_{14}$ coincide as subsets of $\mathbb{C} H^{4}$ (Lemma 12.1) and $T_{1}$ carries $r_{14}$ to a negative multiple of $r_{04}$. Similarly, using the intersections of $P_{1}$ with $P_{2}, P_{2}$ with $P_{3}$, and $P_{3}$ with $P_{4}$ described in Lemma 12.1, we find

$$
\begin{aligned}
& P_{2}=T_{2}\left(C_{2}\right) \text { where } T_{2}:\left(x_{0}, \ldots, x_{4}\right) \mapsto\left(x_{0}, x_{1}, x_{2}, i x_{3}, i x_{4}\right), \\
& P_{3}=T_{3}\left(C_{3}\right) \text { where } T_{3}:\left(x_{0}, \ldots, x_{4}\right) \mapsto\left(x_{0}, x_{1}, i x_{2}, i x_{3}, i x_{4}\right), \text { and } \\
& P_{4}=T_{4}\left(C_{4}\right) \text { where } T_{4}:\left(x_{0}, \ldots, x_{4}\right) \mapsto\left(x_{0}, i x_{1}, i x_{2}, i x_{3}, i x_{4}\right) .
\end{aligned}
$$

For uniformity of notation we define $T_{0}$ to be the identity map. In all cases we have $P_{j k}=T_{j}\left(C_{j k}\right)$; we selected our labelings of the walls of the $P_{j}$ so that this would hold. We write $s_{j k}$ for $T_{j}\left(r_{j k}\right)$, yielding simple roots for the $P_{j}$. Given $r_{j k}$ from Fig. 5.1, $s_{j k}$ is got by replacing $\theta$ by $-\sqrt{3}$ wherever it appears.

Since simple roots for $P_{1}$ are now known, the matrix for the diagram automorphism $S$ can be worked out, yielding

$$
S=\left(\begin{array}{ccccc}
3 & 2 & 1 & 0 & -\sqrt{3} \\
-2 & -1 & -1 & 0 & \sqrt{3} \\
-1 & -1 & -1 & 0 & 0 \\
0 & 0 & 0 & 1 & 0 \\
\sqrt{3} & \sqrt{3} & 0 & 0 & -1
\end{array}\right) .
$$

Since $P_{0}^{\prime}, P_{3}^{\prime}$ and $P_{4}^{\prime}$ are the images of $P_{0}, P_{3}$ and $P_{4}$ under $S$, they are described by simple roots $s_{j k}^{\prime}=S \cdot s_{j k}$. We now have explicit simple roots for all eight chambers comprising $Q$.

To obtain simple roots for $Q$, we take all the $s_{j k}$ and $s_{j k}^{\prime}$ and discard those involved in the gluing of Fig. 12.1, namely $s_{04}, s_{04}^{\prime}, s_{14}, s_{17}, s_{13}, s_{24}, s_{26}, s_{22}, s_{34}, s_{34}^{\prime}, s_{31}, s_{31}^{\prime}, s_{44}$ and $s_{44}^{\prime}$. This leaves us with 36 simple roots. There is a great deal of duplication, for example $s_{01}$ and $s_{43}$ are positive scalar multiples of each other. After eliminating duplicates, only 10 remain, given in table 12.1. We will indicate the walls of $Q$ by $A, \ldots, E, E^{\prime}, \ldots, A^{\prime}$ and corresponding simple roots by $s_{A}, \ldots, s_{E}, s_{E}^{\prime}, \ldots, s_{A}^{\prime}$. We have scaled them so that $s_{A}, s_{B}, s_{B}^{\prime}$ and $s_{A}^{\prime}$ have norm 1 and the rest have norm 2. In the table we also indicate which $P_{j k}$ and $P_{j k}^{\prime}$ lie in each wall of $Q$. The diagram automorphism acts by exchanging primed and unprimed letters. With simple roots in hand, one can work out $Q$ 's dihedral angles, yielding Fig. 12.2 as the Coxeter diagram of $Q$.

\subsection{The subgroup $\frac{1}{2} P \Gamma^{\mathbb{R}}$ for which $Q$ is a fundamental domain}

We already know that $P \Gamma^{\mathbb{R}}$ contains the reflections across $C, D, E, E^{\prime}, D^{\prime}$ and $C^{\prime}$. By Lemma 12.1, $P_{37}$ and $P_{46}$ are identified in $M_{s}^{\mathbb{R}}$, so there exists an element $\tau$ of $P \Gamma^{\mathbb{R}}$ carrying $A=P_{37}$ to $B=P_{46}$. This transformation must carry $P_{3}$ to the type 3 chamber on the other side of $P_{46}$ from $P_{4}$, and so it carries $s_{A}$ to $-s_{B}$. By considering how the walls of $Q$ meet $A$ 


\begin{tabular}{cll} 
root & coordinates & root $^{\perp}$ contains \\
\hline$s_{A}$ & $(3,-1, \sqrt{3}, \sqrt{3}, \sqrt{3})$ & $P_{37}$ \\
$s_{B}$ & $(\sqrt{3}, 1,1,1,1)$ & $P_{46}$ \\
$s_{C}$ & $(1,-1,-1,-1,0)$ & $P_{05}, P_{16}, P_{02}^{\prime}, P_{33}^{\prime}, P_{42}^{\prime}$ \\
$s_{D}$ & $(\sqrt{3},-\sqrt{3}, 0,1,1)$ & $P_{27}, P_{36}, P_{03}^{\prime}, P_{32}^{\prime}, P_{41}^{\prime}$ \\
$s_{E}$ & $(0,1,-1,0,0)$ & $P_{01}, P_{11}, P_{21}, P_{43}, P_{35}^{\prime}, P_{45}^{\prime}$ \\
$s_{E}^{\prime}$ & $(1,0,0,0, \sqrt{3})$ & $P_{15}, P_{25}, P_{35}, P_{45}, P_{01}^{\prime}, P_{43}^{\prime}$ \\
$s_{D}^{\prime}$ & $(0,0,0,1,-1)$ & $P_{03}, P_{23}, P_{32}, P_{41}, P_{36}^{\prime}$ \\
$s_{C}^{\prime}$ & $(0,0,1,-1,0)$ & $P_{02}, P_{12}, P_{33}, P_{42}, P_{05}^{\prime}$ \\
$s_{B}^{\prime}$ & $(3+2 \sqrt{3},-2-\sqrt{3},-2-\sqrt{3}, 1,2+\sqrt{3})$ & $P_{46}^{\prime}$ \\
$s_{A}^{\prime}$ & $(4+\sqrt{3},-2-\sqrt{3},-2-\sqrt{3}, \sqrt{3}, \sqrt{3})$ & $P_{37}^{\prime}$
\end{tabular}

TABLE 12.1. Simple roots for the polyhedron $Q$.

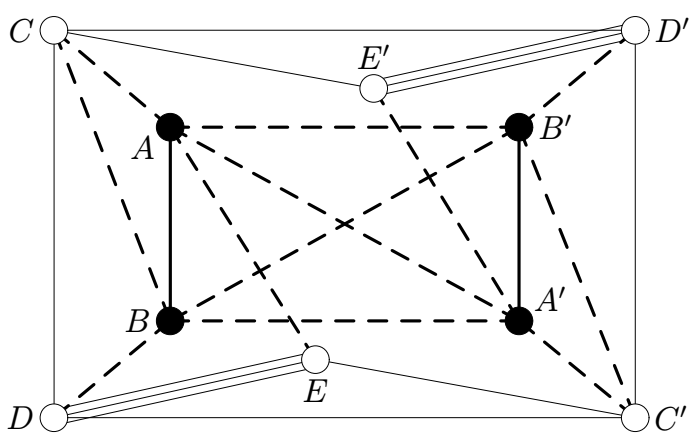

Figure 12.2. The polyhedron $Q$.

and $B$, one sees that $\tau$ must fix each of $s_{E}^{\prime}, s_{D}^{\prime}$ and $s_{C}^{\prime}$, and carry $s_{D}$ to $s_{E}$. This determines $\tau$ uniquely:

$$
\tau=\left(\begin{array}{ccccc}
7+3 \sqrt{3} & 3+\sqrt{3} & -3-2 \sqrt{3} & -3-2 \sqrt{3} & -3-2 \sqrt{3} \\
3+\sqrt{3} & 1 & -1-\sqrt{3} & -1-\sqrt{3} & -1-\sqrt{3} \\
3+2 \sqrt{3} & 1+\sqrt{3} & -1-\sqrt{3} & -2-\sqrt{3} & -2-\sqrt{3} \\
3+2 \sqrt{3} & 1+\sqrt{3} & -2-\sqrt{3} & -1-\sqrt{3} & -2-\sqrt{3} \\
3+2 \sqrt{3} & 1+\sqrt{3} & -2-\sqrt{3} & -2-\sqrt{3} & -1-\sqrt{3}
\end{array}\right) .
$$

Of course, $P \Gamma^{\mathbb{R}}$ also contains $\tau^{\prime}=S \tau S$, which carries $A^{\prime}$ to $B^{\prime}$. We define $\frac{1}{2} P \Gamma^{\mathbb{R}}$ to be the subgroup of $P \Gamma^{\mathbb{R}}$ generated by $\tau, \tau^{\prime}$ and the reflections in $C, D, E, E^{\prime}, D^{\prime}$ and $C^{\prime}$.

Lemma 12.3. - $Q$ is a fundamental domain for $\frac{1}{2} P \Gamma^{\mathbb{R}}$. More precisely, the $\frac{1}{2} P \Gamma^{\mathbb{R}}$-images of $Q$ cover $H \cong H^{4}$, and the only identifications among points of $Q$ under $Q \rightarrow \frac{1}{2} P \Gamma^{\mathbb{R}} \backslash H$ are that $A$ (resp. $\left.A^{\prime}\right)$ is identified with $B$ (resp. $\left.B^{\prime}\right)$ by the action of $\tau$ (resp. $\left.\tau^{\prime}\right)$. 
Proof. - All our claims follow from Poincaré's polyhedron theorem, as formulated in [26, sec. IV.H]. There are 7 conditions to verify. The key points are that any two Eckardt walls that intersect make an angle of the form $\pi$ /(an integer), and that the 4 discriminant walls are disjoint from each other and orthogonal to the Eckardt walls that they meet. These properties dispose of Maskit's conditions (i)-(vi). Condition (vii) is that $Q$ modulo the identifications induced by $\tau$ and $\tau^{\prime}$ is metrically complete. This follows because we already know from Theorem 11.7 that $P \Gamma^{\mathbb{R}} \backslash H^{4}$ is complete, and $\frac{1}{2} P \Gamma^{\mathbb{R}} \subseteq P \Gamma^{\mathbb{R}}$.

The main theorem of this section is now an easy consequence:

TheOrem 12.4. $-P \Gamma^{\mathbb{R}}=\left(\frac{1}{2} P \Gamma^{\mathbb{R}}\right) \rtimes \mathbb{Z} / 2$, the $\mathbb{Z} / 2$ being the diagram automorphism $S$.

Proof. - Because $S$ sends $Q$ to itself, and $Q$ is a fundamental domain for $\frac{1}{2} P \Gamma^{\mathbb{R}}$, $S \notin \frac{1}{2} P \Gamma^{\mathbb{R}}$. Since $S$ normalizes $\frac{1}{2} P \Gamma^{\mathbb{R}}$, we have

$$
\left\langle\frac{1}{2} P \Gamma^{\mathbb{R}}, S\right\rangle=\frac{1}{2} P \Gamma^{\mathbb{R}} \rtimes\langle S\rangle .
$$

Since this larger group lies in $P \Gamma^{\mathbb{R}}$ and has the same covolume as $P \Gamma^{\mathbb{R}}$, it equals $P \Gamma^{\mathbb{R}}$.

REMARK. - Poincarés polyhedron theorem readily gives a presentation for $P \Gamma^{\mathbb{R}}$ : there are generators $C, C^{\prime}, D, D^{\prime}, E, E^{\prime}$ (the reflections in the Eckardt walls of $Q$ ), $\tau, \tau^{\prime}$ (the maps carrying $A$ to $B$, respectively $A^{\prime}$ to $B^{\prime}$ ), and $S$ (the diagram automorphism) with the following relations. (1) The subgroup generated by $C, D, E, C^{\prime}, D^{\prime}, E^{\prime}$ has the Coxeter presentation indicated in the diagram. (2) $\tau$ commutes with $C^{\prime}, D^{\prime}, E^{\prime}$ while $\tau D=E \tau$. (3) The relations obtained from (2) by interchanging the primed and unprimed letters. (4) $S$ is an involution and conjugation by it interchanges all the primed and unprimed generators. A presentation for $\frac{1}{2} P \Gamma^{\mathbb{R}}$ is obtained by deleting the generator $S$ and the relations (4).

\subsection{The discriminant in $\mathcal{M}_{s}^{\mathbb{R}} \cong P \Gamma^{\mathbb{R}} \backslash H^{4}$}

We have now established Theorem 1.2, except for the nonarithmeticity and the fact that $\mathcal{M}_{0}^{\mathbb{R}} \subseteq \mathcal{M}_{s}^{\mathbb{R}}$ corresponds to $P \Gamma^{\mathbb{R}} \backslash\left(H^{4}-\mathcal{H}^{\prime}\right)$ where $\mathcal{H}^{\prime}$ is a union of $H^{2}$ 's and $H^{3}$ 's. We will now address $\mathcal{H}^{\prime}$; see the next section for the nonarithmeticity. The part of $Q$ that lies over the discriminant in $\mathcal{M}_{s}^{\mathbb{R}}$ consists of (1) the walls $A, B, A^{\prime}$ and $B^{\prime}$, (2) the faces corresponding to triple bonds in Fig. 12.2, and (3) the walls of the $P_{j}$ and $P_{j}^{\prime}$ along which we glued the 8 chambers to obtain $Q$. We will refer to a wall of case (3) as an 'interior wall'. Setting $\mathcal{H}^{\prime}$ to be the preimage of $\mathcal{M}_{s}^{\mathbb{R}}-\mathcal{M}_{0}^{\mathbb{R}}$ in $H^{4}$, we see that $\mathcal{H}^{\prime}$ is the union of the $\frac{1}{2} P \Gamma^{\mathbb{R}}$-translates of these three parts of $Q$. The wall $A$ is orthogonal to all the walls of $Q$ that it meets, all of which are Eckardt walls, so it is easy to see that the $H^{3}$ containing $A$ is covered by the $\frac{1}{2} P \Gamma^{\mathbb{R}}$-translates of $A$. The same argument applies with $B, A^{\prime}$ or $B^{\prime}$ in place of $A$, and also applies in case (2), yielding $H^{2}$ 's.

The essential facts for treating case (3) are the following. If $I$ is an interior wall, then every wall $w$ of $Q$ with which $I$ has 2-dimensional intersection is an Eckardt wall of $Q$, and is either orthogonal to $I$ or makes angle $\pi / 4$ with it. In the orthogonal case, it is obvious that $\mathcal{H}^{\prime}$ contains the image of $I$ under reflection across $w$. In the $\pi / 4$ case, one can check that there is another interior wall $I^{\prime}$ with $I^{\prime} \cap w=I \cap w, \angle\left(w, I^{\prime}\right)=\pi / 4$ and $I \perp I^{\prime}$. Then the image of $I^{\prime}$ under reflection across $w$ lies in the same $H^{3}$ as $I$ does. Repeating this process, we see 
that the $H^{3}$ containing $I$ is tiled by $\frac{1}{2} P \Gamma^{\mathbb{R}}$-translates of interior walls of $Q$. It follows that $\mathcal{H}^{\prime}$ is a union of $H^{2}$ 's and $H^{3}$ 's.

We remark that the $H^{3}$ tiled by translates of interior walls can be viewed as a 3-dimensional analogue of our gluing process, describing moduli of real 6-tuples in $\mathbb{C} P^{1}$; see [4] and [3] for details. In particular, its stabilizer in $P \Gamma^{\mathbb{R}}$ is the nonarithmetic group discussed there. Also, see [3] for the 2-dimensional analogue.

\section{Nonarithmeticity}

This section is devoted to proving the following result:

THeOREM 13.1. - $P \Gamma^{\mathbb{R}}$ is a nonarithmetic lattice in $P O(4,1)$.

Our main tool is Corollary 12.2.8 of [11]. We recall the context: $G$ is an adjoint connected absolutely simple non-compact real Lie group, $\mathbf{G}$ is an adjoint connected simple algebraic group over $\mathbb{R}$ so that $G$ is the identity component of $\mathbf{G}(\mathbb{R})$, and $L$ is a lattice in $G$. Let $E=\mathbb{Q}[\operatorname{Tr} \operatorname{Ad} L]$, the field generated over $\mathbb{Q}$ by $\{\operatorname{Tr} \operatorname{Ad} \gamma: \gamma \in L\}$. Assume that there is a totally real number field $F$ and a form $\mathbf{G}_{F}$ of $\mathbf{G}$ over $F$ so that a subgroup of finite index of $L$ is contained in $\mathbf{G}\left(\theta_{F}\right)$, where $\theta_{F}$ is the ring of integers in $F$. It follows that $E \subset F$. With this context in mind, the statement we will use is:

Theorem 13.2 ([11, Corollary 12.2.8]). - A lattice $L \subset G$ is arithmetic in $G$ if and only if for each embedding $\sigma$ of $F$ in $\mathbb{R}$, not inducing the identity embedding of $E$ in $\mathbb{R}$, the real group $\mathbf{G}_{F} \otimes_{F, \sigma} \mathbb{R}$ is compact.

Proof of Theorem 13.1: - To apply Theorem 13.2, we take $G$ to be the connected component of $S O(4,1)$ and $L$ to be the subgroup of $P \Gamma^{\mathbb{R}}$ that acts on $H^{4}$ by orientation-preserving isometries. Note that Isom $H^{4}=\mathrm{PO}(4,1)=\mathrm{SO}(4,1)$, so that $L$ is indeed a subgroup of $G$. We take $\mathbf{G}$ to be the special orthogonal group of the form $\operatorname{diag}\{-1,1,1,1,1\}$ and $F=\mathbb{Q}(\sqrt{3})$. Then $\vartheta_{F}=\mathbb{Z}[\sqrt{3}]$. Note that $\mathbf{G}$ is defined over $\mathbb{Q}$, hence over $F$, and that $L \subset \mathbf{G}(\mathbb{Z}[\sqrt{3}])$. For the last statement we need two observations. First, the matrices (12.1) and (12.2) of $S$ and $\tau$ have entries in $\mathbb{Z}[\sqrt{3}]$. Second, each root $s_{A}, \ldots, s_{E}, s_{E}^{\prime}, \ldots, s_{A}^{\prime}$ in table 12.1 has coordinates in $\mathbb{Z}[\sqrt{3}]$ and norm 1 or 2 ; it follows that the matrix of its reflection has entries in $\mathbb{Z}[\sqrt{3}]$.

Next we show that $E=\mathbb{Q}(\sqrt{3})=F$. It is clear that $E$ is either $\mathbb{Q}$ or $\mathbb{Q}(\sqrt{3})$. To prove that $E=\mathbb{Q}(\sqrt{3})$ it suffices to exhibit a single $\gamma \in L$ with $\operatorname{Tr} \operatorname{Ad} \gamma \notin \mathbb{Q}$. Almost any $\gamma$ will do; we take $\gamma=\left(R_{C} R_{D^{\prime}} R_{E^{\prime}}\right)^{2}$, where the $R$ 's are the reflections in the corresponding simple roots from table 12.1. One can compute a matrix for $\gamma$ and its square and compute their traces, yielding $\operatorname{Tr}(\gamma)=13+6 \sqrt{3}$ and $\operatorname{Tr}\left(\gamma^{2}\right)=209+120 \sqrt{3}$. Since the adjoint representation of $\mathrm{O}(4,1)$ is the exterior square of the standard one, we can use the formula

$$
\operatorname{Tr} \operatorname{Ad}(\gamma)=\frac{1}{2}\left((\operatorname{Tr}(\gamma))^{2}-\operatorname{Tr}\left(\gamma^{2}\right)\right)=34+18 \sqrt{3} \notin \mathbb{Q} .
$$

This proves $E=\mathbb{Q}(\sqrt{3})$.

Finally, if $\sigma$ denotes the non-identity embedding of $F$ in $\mathbb{R}$, then since $E=F$ it does not induce the identity embedding of $E$. Since the form $\operatorname{diag}\{-1,1,1,1,1\}$ defining $\mathbf{G}$ is 
fixed by $\sigma$, the group $\mathbf{G}_{F} \otimes_{F, \sigma} \mathbb{R}$ is again the non-compact group $S O(4,1)$. Thus $L$ is not arithmetic.

REMARKS. - (1) In the introduction we said that our gluing construction is philosophically that of Gromov and Piatetski-Shapiro [15]. But in technical detail it is quite different. They glue hyperbolic manifolds with boundary whose fundamental groups are Zariski dense in $P O(n, 1)$ and lie in non-commensurable arithmetic lattices. We glue orbifolds with boundary and corners whose orbifold fundamental groups are not Zariski dense in $P O(4,1)$ (but do lie in arithmetic lattices that are not all commensurable). In particular, we cannot directly apply their methods to prove non-arithmeticity.

(2) We wonder whether the unimodular lattice $\operatorname{diag}\{-1,1,1,1,1\}$ over $\mathbb{Z}[\sqrt{3}]$ plays some deeper geometric or arithmetic role. For example, $P \Gamma^{\mathbb{R}}$ maps to $\mathrm{PO}\left(5, \mathbb{F}_{3}\right) \cong W\left(E_{6}\right)$ by reduction modulo $\sqrt{3}$. On each component of the smooth moduli space, the action on this $\mathbb{F}_{3}$ vector space is the same as the action on $V$ from Sections 4 and 8 . But it is not clear what this really means.

(3) The group generated by reflections in the facets of $Q$, while being quite different from $P \Gamma^{\mathbb{R}}$, also preserves this $\mathbb{Z}[\sqrt{3}]$-lattice and is also nonarithmetic.

\section{REFERENCES}

[1] D. Allcock, J. A. Carlson, D. Toledo, The complex hyperbolic geometry of the moduli space of cubic surfaces, J. Algebraic Geom. 11 (2002), 659-724.

[2] D. Allcock, J. A. Carlson, D. Toledo, Real cubic surfaces and real hyperbolic geometry, C. R. Math. Acad. Sci. Paris 337 (2003), 185-188.

[3] D. Allcock, J. A. Carlson, D. Toledo, Nonarithmetic uniformization of some real moduli spaces, Geom. Dedicata 122 (2006), 159-169 and erratum 171.

[4] D. Allcock, J. A. Carlson, D. Toledo, Hyperbolic geometry and the moduli space of real binary sextics, in Algebra and Geometry around Hypergeometric Functions (R. P. Holzapfel, A. M. Uludag, M. Yoshida, eds.), Progress in Math., Birkhäuser, 2007.

[5] F. ApÉRY, M. Yoshida, Pentagonal structure of the configuration space of five points in the real projective line, Kyushu J. Math. 52 (1998), 1-14.

[6] M. R. Bridson, A. Haefliger, Metric spaces of non-positive curvature, Grund. Math. Wiss. 319, Springer, 1999.

[7] J. W. Bruce, C. T. C. Wall, On the classification of cubic surfaces, J. London Math. Soc. 19 (1979), 245-256.

[8] K. CHU, On the geometry of the moduli space of real binary octics, to appear.

[9] J. H. Conway, R. T. Curtis, S. P. Norton, R. A. Parker, R. A. Wilson, Atlas of finite groups, Oxford Univ. Press, 1985.

[10] A. Degtyarev, I. Itenberg, V. M. Kharlamov, Real Enriques surfaces, Lecture Notes in Math. 1746, Springer, 2000.

[11] P. Deligne, G. D. Mostow, Monodromy of hypergeometric functions and nonlattice integral monodromy, Publ. Math. I.H.É.S. 63 (1986), 5-89. 
[12] S. Finashin, V. M. Kharlamov, Deformation classes of real four-dimensional cubic hypersurfaces, J. Algebraic Geom. 17 (2008), 677-707.

[13] S. Finashin, V. M. Kharlamov, On the deformation chirality of real cubic fourfolds, preprint arXiv:0804.4882.

[14] M. Goresky, R. MacPherson, Stratified Morse theory, Ergebnisse Math. Grenzg. (3) 14, Springer, 1988.

[15] M. Gromov, I. Piatetski-Shapiro, Nonarithmetic groups in Lobachevsky spaces, Publ. Math. I.H.É.S. 66 (1988), 93-103.

[16] B. H. Gross, J. Harris, Real algebraic curves, Ann. Sci. École Norm. Sup. 14 (1981), 157-182.

[17] P. DE LA HARPE, An invitation to Coxeter groups, in Group theory from a geometrical viewpoint (Trieste, 1990), World Sci. Publ., River Edge, NJ, 1991, 193-253.

[18] D. Hilbert, Über die volle Invariantensysteme, Math. Annalen 42 (1893), 313-370, English transl. Hilbert's invariant theory papers in Lie Groups: History, Frontiers and Applications, VIII, Math Sci Press, Brookline, Mass., 1978.

[19] V. M. Kharlamov, Topological types of non-singular surfaces of degree 4 in $R \mathrm{P}^{3}$, Funct. Anal. Appl. 10 (1976), 55-68.

[20] V. M. Kharlamov, On the classification of nonsingular surfaces of degree 4 in $\mathbf{R P}^{3}$ with respect to rigid isotopies, Funktsional. Anal. i Prilozhen. 18 (1984), 49-56.

[21] F. KLeIn, Über Flächen dritter Ordnung, Math. Ann. 6 (1873), 551-581, also in Gesammelte Mathematische Abhandlungen, II, 11-62, Springer, 1922.

[22] V. A. Krasnov, Rigid isotopy classification of real three-dimensional cubics, Izv. Math. 70 (2006), 731-768.

[23] R. LazA, The moduli space of cubic fourfolds, J. Algebraic Geom. 18 (2009), 511-545.

[24] E. Looijenga, The period map for cubic fourfolds, Invent. Math 177 (2009), 213-233.

[25] Y. I. Manin, Cubic forms, second ed., North-Holland Mathematical Library 4, NorthHolland Publishing Co., 1986.

[26] B. Maskit, Kleinian groups, Grund. Math. Wiss. 287, Springer, 1988.

[27] S. Moriceau, Surfaces de degré 4 avec un point double non dégénéré dans l'espace projectif réel de dimension 3, Thèse de doctorat, Université de Rennes I, 2004.

[28] I. Newton, Enumeratio linearum tertii ordinis, in Opticks, 1704, 139-162.

[29] I. Newton, The mathematical papers of Isaac Newton. Vol. II: 1667-1670, edited by D. T. Whiteside, Cambridge Univ. Press, 1968.

[30] V. V. NikUlin, Integral symmetric bilinear forms and some of their applications, Math. USSR Izvestiya 14 (1980), 103-167.

[31] L. SCHLÄFLI, An attempt to determine the twenty-seven lines upon a surface of the third order, and to divide such surfaces into species in reference to the reality of the lines upon the surface, Quart. J. Math. 2 (1858), 55-65 and 110-120, also in Gesammelte Mathematische Abhandlungen, II, 198-216, Birkhäuser, 1953.

[32] L. SCHLÄFLI, On the distribution of surfaces of the third order into species, in reference to the absence or presence of singular points, and the reality of their lines, Philos. Trans. Roy. Soc. London 153 (1863), 193-241, also in Gesammelte Mathematische Abhandlungen, II, 304-360, 1953.

$4^{\text {e }}$ SÉRIE - TOME $43-2010-\mathrm{N}^{\mathrm{o}} 1$ 
[33] L. SCHLÄFLI, Quand'è che dalla superficie generale di terzo ordine si stacca una patre che non sia realmente segata a ogni piano reale?, Ann. Mat. pura appl. 5(1871-1873), 289-295, Corezzioni, ibid. 7 (1875/76), 193-196, also in Gesammelte Mathematische Abhandlungen, III, 229-234, 235-237, Birkhäuser, 1956.

[34] B. Segre, The Non-singular Cubic Surfaces, Oxford Univ. Press, 1942.

[35] W. P. Thurston, The geometry and topology of three-manifolds, Princeton University notes, http://www.msri.org/publications/books/gt3m, 1980.

[36] W. P. Thurston, Shapes of polyhedra and triangulations of the sphere, in The Epstein birthday schrift, Geom. Topol. Monogr. 1, Coventry, 1998, 511-549.

[37] E. B. VinBERG, Discrete linear groups that are generated by reflections, Izv. Akad. Nauk SSSR Ser. Mat. 35 (1971), 1072-1112.

[38] E. B. Vinberg, The groups of units of certain quadratic forms, Mat. Sb. (N.S.) 87 (1972), 17-35.

[39] E. B. Vinberg, Some arithmetical discrete groups in Lobačevskiǐ spaces, in Discrete subgroups of Lie groups and applications to moduli (Internat. Colloq., Bombay, 1973), Oxford Univ. Press, 1975, 323-348.

[40] M. Yoshida, The real loci of the configuration space of six points on the projective line and a Picard modular 3-fold, Kumamoto J. Math. 11 (1998), 43-67.

[41] M. Yoshida, A hyperbolic structure on the real locus of the moduli space of marked cubic surfaces, Topology 40 (2001), 469-473.

\footnotetext{
Daniel AllCock

Department of Mathematics

University of Texas at Austin

Austin, TX 78712, USA

E-mail: allcock@math.utexas.edu

URL: http://www.math.utexas.edu/ allcock

James A. CARLSON

Department of Mathematics

University of Utah

Salt Lake City, UT 84112, USA

Clay Mathematics Institute One Bow Street

Cambridge, MA 02138, USA

E-mail: carlson@claymath.org

URL: http://www.math.utah.edu/ carlson

Domingo Toledo

Department of Mathematics

University of Utah

Salt Lake City, UT 84112, USA

E-mail: toledo@math.utah.edu

URL: http://www.math.utah.edu/ toledo
} 